\title{
ASSESSING THE USEFULNESS OF THE AUTOMATED MONITORING SYSTEMS ECOTOX AND DAPHNIATOX IN AN INTEGRATED EARLY-WARNING SYSTEM FOR DRINKING WATER
}

\author{
By Isabelle Netto \\ Honours Bachelor of Science in Zoology, University of Toronto, 2006 \\ A Thesis \\ Presented to Ryerson University \\ In partial fulfillment of the \\ Requirements for the degree of \\ Master of Applied Science \\ In the Program of \\ Environmental Applied Science and Management
}

Toronto, Ontario, Canada, 2010

(C) Isabelle Netto, 2010 
I hereby declare that I am the sole author of this thesis.

I authorize Ryerson University to lend this thesis or dissertation to other institutions or individuals for the purpose of scholarly research.

\title{
Isabelle Netto
}

I further authorize Ryerson University to reproduce this thesis by photocopying or by other means, I total or in part, at the request of other institutions or individuals for the purpose of scholarly research.

\author{
Isabelle Netto
}


Isabelle Netto

Master of Applied Science

Environmental Applied Science and Management

August 2010

Ryerson University

\begin{abstract}
Assessing the usefulness of the automated monitoring systems ECOTOX and DaphniaTox in an integrated early-warning system for drinking water.

This study assesses the suitability of the behavioural image analysis systems ECOTOX and DaphniaTox for inclusion in an integrated early-warning system for drinking water quality to be implemented in Canada. Results of behavioural parameters measured by ECOTOX using Euglena gracilis are compared to visual observations of $E$. gracilis behaviour after exposure to atrazine, tributyltin, and copper to determine the automated system's sensitivity. The usability of the DaphniaTox automated system is assessed using the aquatic macroinvertebrate species Daphnia magna and Hyalella azteca. The possible use of the rotifers Brachionus calyciflorus and Brachionus havanensis with the ECOTOX system is also assessed. Findings indicate that at the present state ECOTOX and DaphniaTox are not suitable for inclusion in an early-warning system, but based on visual observation the parameters measured are sensitive to the contaminants tested and consistent, and with suggested modifications these systems have the potential to be fitting additions in an early-warning system.
\end{abstract}




\section{Acknowledgements}

I would like to thank Dr. Vadim Bostan, my supervisor, for his tireless encouragement and motivation throughout these two years, for always being involved and providing guidance and support, and motivation and inspiration at every step of the way. His dedication and patience went a long way to make this possible. I would also like to thank Dr. Lynda McCarthy for her invaluable input and enthusiasm for the project, and Dr. Andrew Laursen for extensive technical support and data analysis support.

I am especially grateful to Karen Terry for being extremely helpful and willing to provide support for most, if not all, lab undertakings, and making it all look easy to boot. I am also grateful to my fellow graduate students, you are colleagues and friends. Thank you Alexia Lane, Vivian Fleet and Andrea Dort for listening, sharing ideas, support, fun, and all the rest. I'd also like to thank Jason Solnik for being a constant source of help and humour in the lab.

Finally, I would like to thank my family for all your encouragement and patience, I am so happy I can share this with you. I am especially grateful to my father, George, for caring so much and making this possible in so many ways; to my mother, Julie, for being also a friend and knowing me as well as you do, we may be far but I always feel that you're near me; and to my stepfather, Stefan, thank you for all your support. 


\section{Contents}

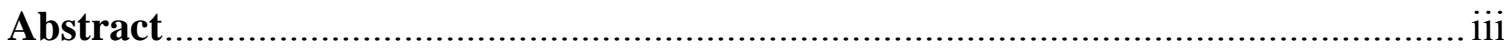

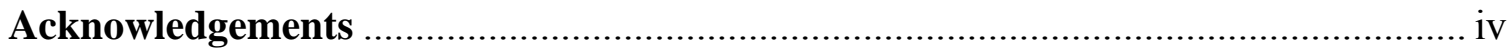

List of Figures.................................................................................................. vii

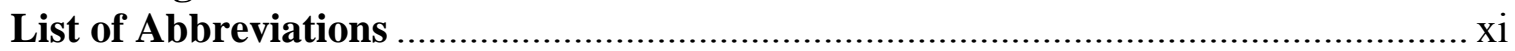

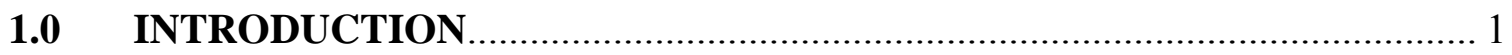

$1.1 \quad$ Water quality in the Great Lakes region ...................................................... 3

$1.2 \quad$ Importance of biological monitoring ........................................................ 5

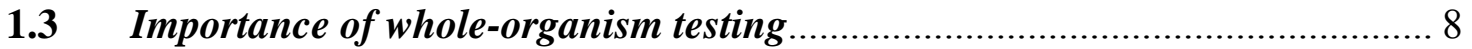

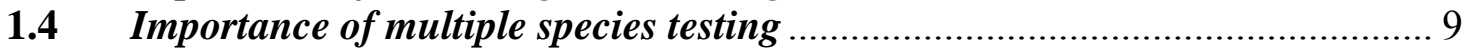

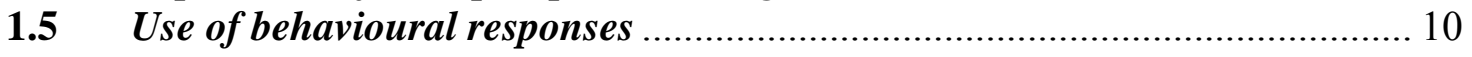

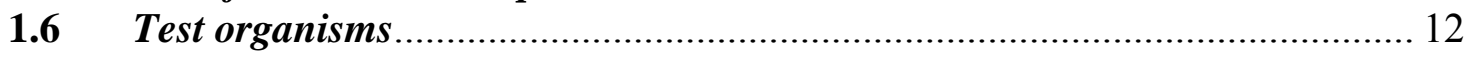

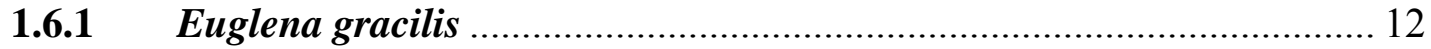

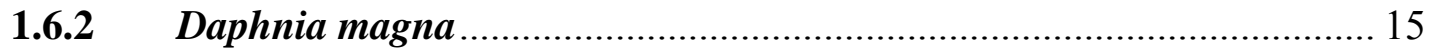

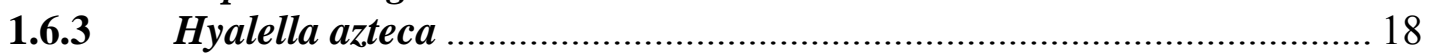

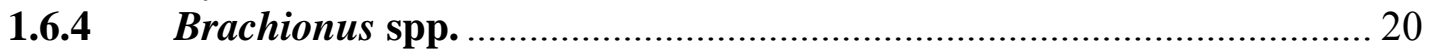

$1.7 \quad$ Automated Biomonitoring Systems …………............................................ 22

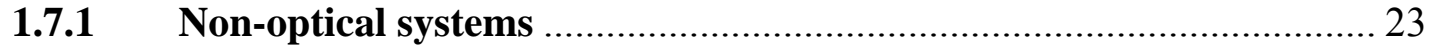

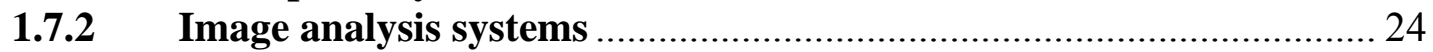

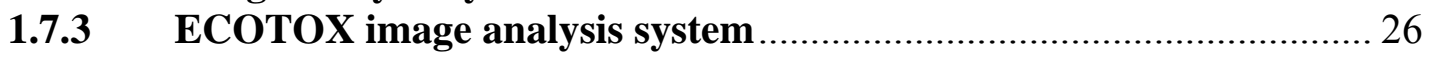

1.7.4 DaphniaTox image analysis system ………….................................. 32

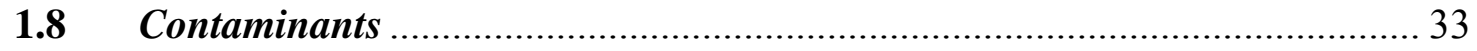

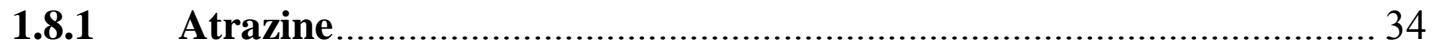

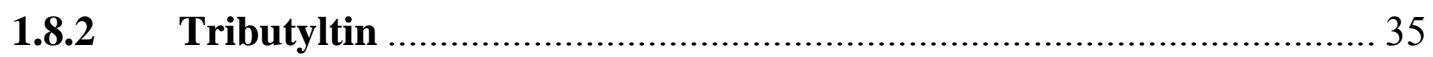

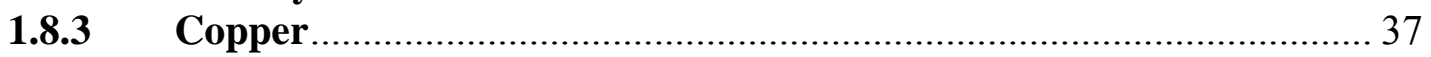

1.8.4 DMSO

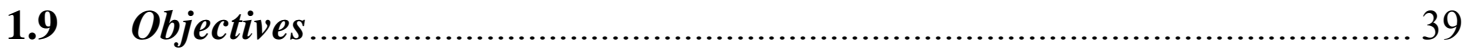

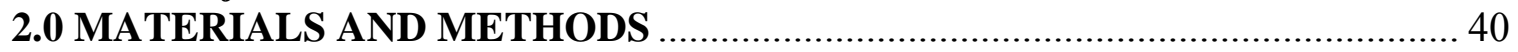

2.1 Culturing of Euglena gracilis ........................................................................ 40

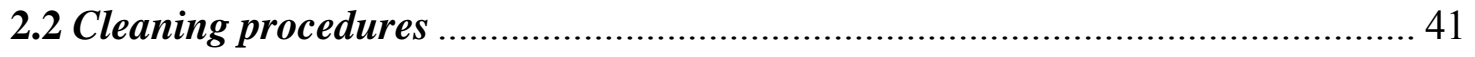

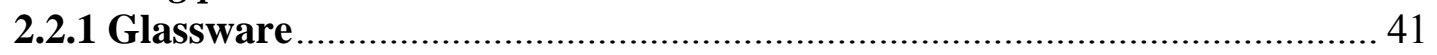

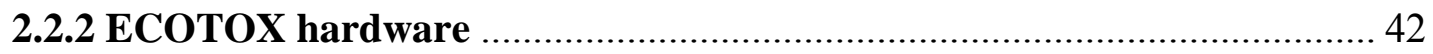

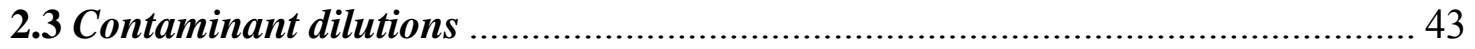

2.4 Euglena gracilis cell motility experiments - visual observation .......................... 43

2.5 Euglena gracilis cell shape experiments - visual observation .............................. 46

2.6 ECOTOX automated system experiments ....................................................... 47

2.6.1 Hardware setup …………………...................................................... 47

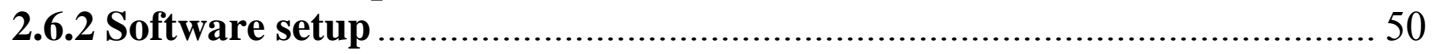

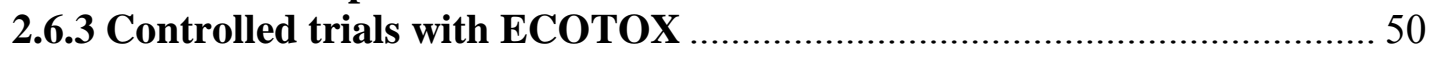

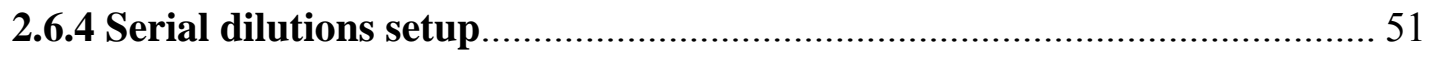

2.6.5 Standard dilutions and incubation setup …………................................... 54

2.7 Culturing of Daphnia magna..................................................................... 55

2.8 Culturing of Hyalella azteca ......................................................................... 56

2.9 Culturing of Pseudokirchneriella subcapitata for invertebrate feeding ............ 57

2.10 DaphniaTox automated system experiments ................................................. 58

2.10.1 Hardware and software setup ............................................................... 58 


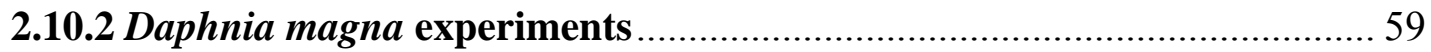

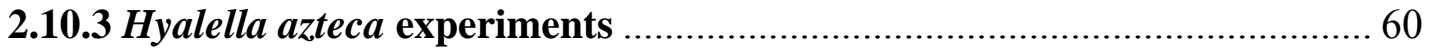

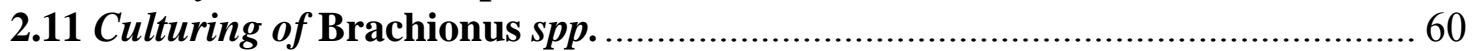

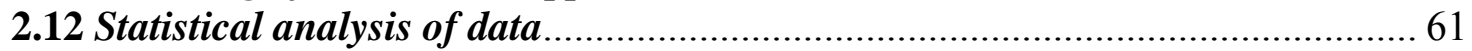

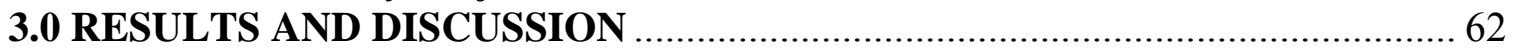

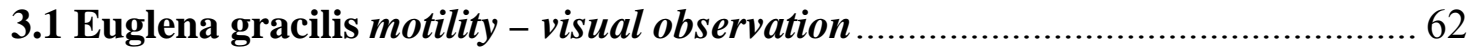

3.1.1 Euglena gracilis motility after atrazine exposure ...................................... 62

3.1.2 Euglena gracilis motility after tributyltin exposure................................... 64

3.1.3 Euglena gracilis motility after copper exposure......................................... 65

3.1.3. a Effects of temperature on copper toxicity in Euglena gracilis .................. 67

3.1.3.b. Effects of water hardness on copper toxicity in Euglena gracilis ............ 69

3.2 Euglena gracilis cell shape - visual observation .............................................. 70

3.3 Euglena gracilis automated parameters measured with ECOTOX ……............. 71

3.3.1 Euglena gracilis automated parameters after atrazine exposure .............. 72

3.3.1.a. Gravitactic behaviour and atrazine exposure ....................................... 72

3.3.1.b. Velocity and atrazine exposure ………………..................................... 75

3.3.1.c. Cell shape and atrazine exposure ......................................................... 75

3.3.1.d. $R$-value and atrazine exposure .......................................................... 76

3.3.2 Euglena gracilis automated parameters after TBT exposure …………..... 76

3.3.2.a. Gravitactic behaviour and TBT exposure ............................................. 76

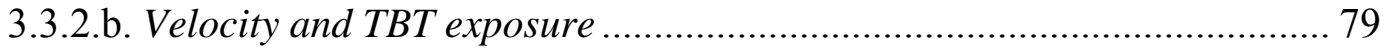

3.3.2.c. Cell shape and TBT exposure ........................................................... 79

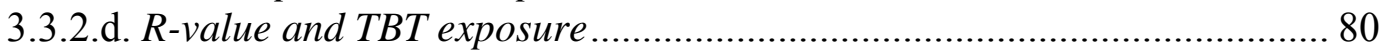

3.3.3 Euglena gracilis automated parameters after copper exposure ……......... 81

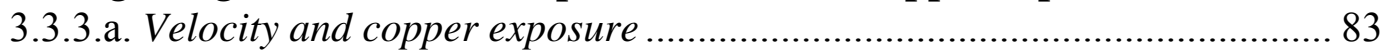

3.3.3.b. Gravitactic behaviour and copper exposure............................................ 84

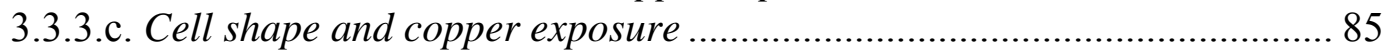

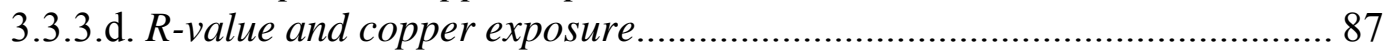

3.4.1 Behavioural parameter: cell swimming velocity ……….......................... 87

3.4.2 Behavioural parameter: gravitactic orientation ....................................... 90

3.4.3 Behavioural parameter: cell shape ……………...................................... 92

3.4.4 Behavioural parameter: randomness of swimming …………………....... 94

3.4.5 ЕСОTOX hardware and software stability............................................ 95

3.4.5.a. Software and hardware maintenance...................................................... 96

3.4.5.b. Quality of ECOTOX manual and technical support ................................. 97

3.4.5.c. Weekly upkeep for water quality testing .................................................. 97

3.4.5.d. Resilience and sensitivity of the ECOTOX system ................................. 98

3.5 Suitability of the automated image analysis system DaphniaTox........................ 99

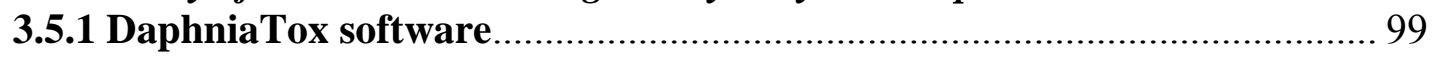

3.5.2 DaphniaTox parameters....................................................................... 100

3.6 Case study: implementation of an image analysis system in an early-warning system for drinking water in Zurich, Switzerland ................................................ 101

3.7 Importance of implementation of an early-warning system for monitoring

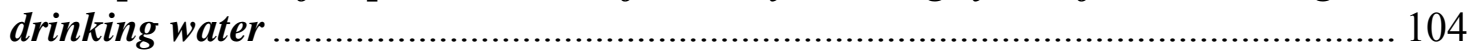

3.8 Suitability of Brachionus spp. as test organisms ............................................. 105

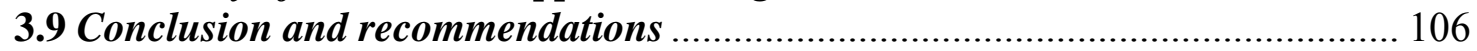




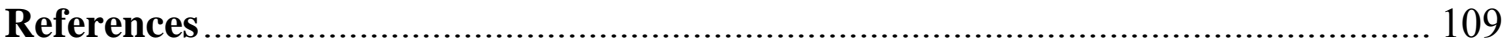

Appendices......

Appendix A - Nutrient concentration of liquid mineral medium for E. gracilis culturing.

Appendix B - Calculation of water hardness for Daphnia magna culture water and Euglena gracilis culture medium.

Appendix C - Nutrient concentration of liquid growth medium for P. subcapitata culturing.

Appendix D - Nutrient concentration of COMBO growth medium for culturing of rotifers.

Appendix E - Euglena gracilis percent motility after atrazine exposure - visual observation.

Appendix F - Euglena gracilis percent motility after TBT exposure - visual observation.

Appendix G - Euglena gracilis percent motility after copper exposure - visual observation.

Appendix $\mathbf{H}$ - Euglena gracilis percent motility after copper exposure at different temperatures - visual observation.

Appendix I - Euglena gracilis cell shape after copper exposure

Appendix $\mathbf{J}$ - Euglena gracilis automated parameters measured by ECOTOX after atrazine exposure.

Appendix K - Euglena gracilis automated parameters measured by ECOTOX after TBT exposure.

Appendix L - Euglena gracilis automated parameters measured by ECOTOX after copper exposure. 


\section{List of Figures}

Figure 1.1. Euglena gracilis.

Figure 1.2. Daphnia magna.

Figure 1.3. Image analysis tracking of swimming trajectory of Daphnia magna.

Figure 1.4. Hyalella azteca.

Figure 1.5. Brachionus havanensis.

Figure 1.6. Brachionus calyciflorus.

Figure 1.7. Diagram of German-made bbe Daphnia Toximeter.

Figure 1.8. Schematic diagram of the ECOTOX Image Analysis System.

Figure 1.9. Example of a circular histogram in ECOTOX.

Figure 1.10. Percentage of upwards-swimming cells, r-values, and circular histograms of Euglena gracilis behaviour in the absence of contaminants.

Figure 1.11. Dose-response curves built using the ECOTOX image analysis system.

Figure 1.12. The DaphniaTox image analysis system.

Figure 2.1. Experimental setup for cell motility experiments.

Figure 2.2. The ECOTOX hardware system

Figure 2.3. Proportional diagram of volumes of cell suspension

Figure 2.4. ECOTOX software screenshot.

Figure 2.5. Experimental setup for the ECOTOX measurement experiments.

Figure 2.6. DaphniaTox software screenshot 
Figure 3.1. Cell motility of E. gracilis after $1 \mathrm{~h}, 2 \mathrm{~h}$, and $24 \mathrm{~h}$ exposure to atrazine.

Figure 3.2. Cell motility of E. gracilis after $1 \mathrm{~h}, 2 \mathrm{~h}$, and $24 \mathrm{~h}$ exposure to tributyltin.

Figure 3.3. Cell motility of E. gracilis after $1 \mathrm{~h}, 2 \mathrm{~h}$, and $24 \mathrm{~h}$ exposure to copper. Asterisks indicate a statistically significant difference from reference conditions.

Figure 3.4. Cell motility of E. gracilis after $1 \mathrm{~h}$, and $2 \mathrm{~h}$ exposure to copper at $21^{\circ} \mathrm{C}$ and $26^{\circ} \mathrm{C}$.

Figure 3.5. Euglena gracilis cell shape under copper stress after $1 \mathrm{~h}$ and $2 \mathrm{~h}$ incubation.

Figure 3.6. Euglena gracilis cell velocity after incubation in atrazine in 23.2, 231.8, and $2318.2 \mathrm{nM}$.

Figure 3.7. Euglena gracilis gravitactic orientation after incubation in atrazine in 23.2, 231.8, and $2318.2 \mathrm{nM}$.

Figure 3.8. Euglena gracilis cell shape (decreasing numbers represent change towards cyst-form) after incubation in atrazine in 23.2, 231.8, and $2318.2 \mathrm{nM}$.

Figure 3.9. Euglena gracilis randomness of swimming (decreasing numbers represent more random swimming) after incubation in atrazine in 23.2, 231.8, and $2318.2 \mathrm{nM}$.

Figure 3.10. Euglena gracilis cell velocity after incubation in TBT in 1.7, 16.8, and $167.9 \mathrm{nM}$.

Figure 3.11. Euglena gracilis gravitactic orientation after incubation in TBT in 1.7, 16.8, and $167.9 \mathrm{nM}$.

Figure 3.12. Euglena gracilis cell shape (decreasing numbers represent change towards cyst-form) after incubation in TBT in $1.7,16.8$, and $167.9 \mathrm{nM}$.

Figure 3.13. Euglena gracilis randomness of swimming (decreasing numbers represent more random swimming) after incubation in TBT in $1.7,16.8$, and $167.9 \mathrm{nM}$.

Figure 3.14. Euglena gracilis cell velocity after incubation in copper in $0.4,1.2,2$, and $20 \mu \mathrm{M}$.

Figure 3.15. Euglena gracilis gravitactic orientation after incubation in copper in 0.4 , $1.2,2$, and $20 \mu \mathrm{M}$. 
Figure 3.16. Euglena gracilis cell shape (decreasing numbers represent change towards cyst-form) after incubation in copper in $0.4,1.2,2$, and $20 \mu \mathrm{M}$.

Figure 3.17. Euglena gracilis randomness of swimming (decreasing numbers represent more random swimming) after incubation in copper in $0.4,1.2,2$, and $20 \mu \mathrm{M}$.

Figure 3.18. Euglena gracilis swimming velocity measured in $\mu \mathrm{m} / \mathrm{s}$ after 5 minutes exposure to several controls and in comparison to readings from an empty cuvette.

Figure 3.19. Euglena gracilis gravitactic behaviour (measured in percent cells swimming up) after 5 minutes exposure to several controls and in comparison to readings from an empty cuvette.

Figure 3.20. Euglena gracilis cell shape (measured in form factor) after 5 minutes exposure to several controls and in comparison to readings from an empty cuvette.

Figure 3.21. Euglena gracilis randomness of swimming (measured in r-value) after 5 minutes exposure to several controls and in comparison to readings from an empty cuvette.

Figure 3.22. The calculation of parameters on ECOTOX.

Figure 3.23. Laboratory at the Hardhof groundwater plant in Zurich, Switzerland.

Figure 3.24. The bbe Daphnia Toximeter in use at the Hardhof groundwater plant in Zurich, Switzerland. 


\section{List of Abbreviations}

ANOVA - analysis of variance

DMSO - Dimethylsulfoxide

$\mathbf{E C}_{50}$ - Effective concentration at which the response is present for $50 \%$ of the population

IJC - International Joint Commission

$\mathbf{K}_{\mathbf{o w}}$ - Log octanol-water partition coefficient

$\mathbf{L C}_{50}$ - Effective concentration inducing 50\% lethality in a group of test organisms

LOEC - Lowest Observable Effect Concentration

MFB - Multispecies Freshwater Biomonitor

NOEC - No Observable Effect Concentration

NPCA - Niagara Peninsula Conservation Authority

PAM - Pulse-Amplitude-Modulation

PBDE - Polybrominated diphenyl ether

PFB - Paraflagellar body

PMRA - Health Canada's Pest Management Regulatory Agency

ppm - parts per million

SDWA - Ontario Safe Drinking Water Act of 2002

TBT - tributyltin

WHO - World Health Organization 


\subsection{INTRODUCTION}

This study investigates the suitability of the automated behavioural image analysis systems ECOTOX and DaphniaTox when used with the freshwater species Euglena gracilis, a photosynthesizing flagellate, Daphnia magna, a cladoceran also known as the water flea, Hyalella azteca, a benthic amphipod, and Brachionus calyciflorus and Brachionus havanensis, planktonic rotifers. The goal of this study is to determine the applicability of these automated systems within an early-warning drinking water monitoring system. Results of this study will complement a larger project at Ryerson University which aims to develop an early-warning system for monitoring drinking water quality and to implement this system within a drinking water distribution plant in the Niagara region in Southern Ontario. Several characteristics of this proposed system provide specific advantages with respect to implementation in a water quality testing facility, which are summarized in table 1.1.

The term "miner's canary" comes from the practice of taking birds into mines to observe their reaction as a test for air quality and to ensure safe levels of carbon monoxide before miners entered the mine. The early-warning system in development by this project would act as a "miner's canary" in that it would provide information from biological responses of organisms to toxicants which may be present in the water before it reaches the public. In addition, this system would be based on responses of a variety of organisms within the aquatic ecosystem, including plants, algae, and invertebrates, each of which respond to contaminants in different ways and with different sensitivities. By building on a database of responses of these organisms exposed to different contaminants, it may be possible to determine the identity or the category of contaminant by matching the pattern of responses obtained while the system is in use to the pattern of responses obtained in the laboratory after exposing organisms to different contaminants. In this way the early-warning system being developed for this project will provide an additional advantage over other single-species based systems, to create a final drinking water monitoring system which will be sensitive to contaminants, robust, reliable, 
automated, and provide complete, immediate, and environmentally relevant information about potential toxicants in the drinking water being tested.

Table 1.1. Advantages of implementing the early-warning system for monitoring drinking water quality being developed at Ryerson University.

\begin{tabular}{|c|c|}
\hline Characteristic & Advantage \\
\hline Biomonitoring, no chemical analysis & $\begin{array}{ll}\text { - } & \text { Time } \\
\text { - } & \text { Cost } \\
\text { - } & \text { Environmental relevance }\end{array}$ \\
\hline Whole-organism tests & $\begin{array}{l}\text { - Balance of fast response \& } \\
\text { Environmental relevance } \\
\text { - } \text { Cost }\end{array}$ \\
\hline Multiple species & $\begin{array}{l}\text { - Environmental relevance } \\
\text { - Overall sensitivity of system } \\
\text { - Complete toxicity information } \\
\text { - Potential to categorize contaminant }\end{array}$ \\
\hline Automated & $\begin{array}{l}\text { - Minimal supervision } \\
\text { - Cost } \\
\text { - Ease of implementation }\end{array}$ \\
\hline Choice of organisms based on research & $\begin{array}{l}\text { - } \text { Overall sensitivity of system } \\
\text { - } \text { Minimize error } \\
\text { - } \text { Minimize false positives } \\
\text { - } \text { Minimize variability } \\
\text { - Minimize cost }\end{array}$ \\
\hline Operates in real-time & $\begin{array}{l}\text { - Immediate responses } \\
\text { - Prevents toxicants from reaching } \\
\text { end-users }\end{array}$ \\
\hline Behavioural analysis & $\begin{array}{l}\text { - Visible response } \\
\text { - Sublethal endpoint } \\
\text { - High sensitivity to contaminants } \\
\text { - Wide variety of responses } \\
\text { - Potential to categorize contaminant }\end{array}$ \\
\hline
\end{tabular}

While this study is assessing the use of the ECOTOX and DaphniaTox automated systems, as part of this project Pearce (2009) and Marshall (2009) have investigated the suitability of the test species Lemna minor (duckweed), Pseudokirchneriella subcapitata 
(green alga previously known as Selenastrum capricornutum), Anodonta grandis (mussel), E. gracilis; and D. magna, H. azteca, and Lumbriculus variegatus (blackworm) respectively. Currently as part of this project, the suitability of the automated non-optical behavioural analysis system Multispecies Freshwater Biomonitor, or MFB, is also being assessed by V. Fleet.

\subsection{Water quality in the Great Lakes region}

Twenty-four million people receive their drinking water directly from the great lakes (Findlay \& Telford, 2006; Pelaez et al., 2010). For over a century, the safety of drinking water has been a concern in this region. Typhoid epidemics in the late $19^{\text {th }}$ and early $20^{\text {th }}$ century led to water quality investigations which found that thousands of deaths were caused by these waterborne bacteria, most notably in urban centers such as Chicago (National Research Council, 1985; Bogue, 2000). This and other issues led to the creation of the International Joint Commission (IJC) as part of the Boundary Waters Treaty established in 1909. While investigating waterborne diseases in the Great Lakes, the IJC found in 1914 that the death rate in Sault Sainte Marie, Ontario, was as high as 330 per 100,000 inhabitants due to typhoid (Bogue, 2000). After an IJC report in 1918, chlorination practices were adopted by municipalities and typhoid was largely eradicated, although other water pollution issues remained due to the lack of effluent treatment (Safe Drinking Water Committee, 1980). A second extensive investigation by the IJC was published in 1951 and now included not only bacteriological problems but also industrial chemical pollution, which were starting to be considered a major problem, and included phenols, oils, cyanides, and biological oxygen demand (BOD) (National Research Council, 1985). On April 15, 1972, the Great Lakes Water Quality Agreement was signed in Ottawa by U.S. President Nixon and Canadian Prime Minister Trudeau. This agreement was revised and signed again in 1978, where it called for the discharge of toxic chemicals to be eliminated, and listed 350 "hazardous polluting substances" to be banned from the Great Lakes (National Research Council, 1985). 
Recently, concerns over the quality of drinking water have continued to grow, especially in Canada after recent cases of water-borne outbreaks (Danilov \& Ekelund, 2001; Turgeon et al., 2004; Li et al., 2009). The Escherichia coli pathogen outbreak in Walkerton, Ontario led to a report by Justice Dennis O'Connor which highlighted the importance of a multi-level approach where sources of drinking water should also be protected (O'Connor, 2002; Findlay \& Telford, 2006). The report specifically recommends that:

The next set of barriers to the contamination of drinking water relies on having in place effective standards and technology for treating water and for monitoring its quality as it makes its way to the consumer. I recommend that Ontario's standards and technology be continually updated according to the most recent knowledge and experience. - Justice O'Connor (2002).

This recommendation pertains directly to this project for implementing an early warning system for drinking water quality monitoring in Ontario, as it provides new and updated technology for monitoring water quality that is superior to technologies and methods currently in place, as will be discussed in section 1.2. Recent concerns over drinking water are of course not limited to illness-causing pathogens, but extend to industrial and agricultural runoff and spills (Danilov \& Ekelund, 2001; Li et al., 2009), cyanobacterial blooms (Paez et al., 2010), and new and emerging contaminants such as pharmaceuticals, personal care products, brominated diphenyl ethers (PBDEs), currently used pesticides, and others (McCarthy, 2007; IJC, 2009). Other jurisdictions outside of Canada, such as Zurich, Switzerland, include malicious spills as a factor of concern for monitoring the supply of drinking water (Grand et al., n.d.; R. Engi, personal communication, May 2010). According to the IJC (2006), since September 11, 2001, this is also of potential concern in the Great Lakes region. 
In Ontario, Justice O'Connor's recommendations were incorporated in the Ontario Safe Drinking Water Act of 2002 (SDWA), which created many new standards, including standards for drinking water quality and quality monitoring. An important part of the provision of drinking water is its testing and monitoring. An optimal drinking water quality testing system is one that can provide a safeguard for human health by being effectively implemented and producing reliable results. One weakness in the

present methods of drinking water quality assessment in the Ontario Drinking Water Quality Standards is the limitation to chemical testing of the substances listed by the Safe Drinking Water Act of 2002 (Government of Ontario, 2010). Chemical testing is costly and results may be slow (Green et al., 2003), whereas a complete drinking water monitoring system should provide fast, reliable, and environmentally relevant responses and be able to deem the water safe before it reaches the public, in addition to being sensitive to contaminants and fully automated and able to operate under minimal supervision.

\subsection{Importance of biological monitoring}

This study discusses how biological testing, using automated systems that run continuously and assess sublethal toxicity, can make drinking water monitoring systems more complete by providing biologically relevant, immediate, reliable, and cost-effective information about the safety of drinking water. Implementing an early-warning biomonitoring system consisting of automated assays with several test species having a wide array of biological responses to different contaminants will serve as a robust "miner's canary" assessment and ensure that the water reaching the public is safe to drink and the risk of contamination is minimized. The suitability of two automated systems will be investigated in particular for implementation in a biological drinking water monitoring system The suitability of five species to be used with these automated systems will be explored as well. 
Biomonitoring methods have long been used to evaluate the impact of toxicants in the environment, both in terms of how much exposure to toxicants cause adverse effects and what these impacts might be at all ecological levels (Landis \& Yu, 1995; Kettrup \& Marth, 1998; Gerhardt et al., 2006b; Mikol et al., 2007). Biomonitoring is as old as agriculture and involves the use of an organism to obtain information about the quality of its environment (Kettrup \& Marth, 1998; Kieu et al., 2001; Allan et al., 2006; Gerhardt et al., 2006b;). These organisms, such as the miner's canary, are bioindicators. Biological tests have been used for several decades in Europe to monitor surface waters such as rivers and lakes (LAWA, 1996; Mikol et al., 2007). More recently, biomonitoring systems have been put in place to determine drinking water safety directly (LAWA, 1996; Michels et al., 1999; Lechelt et al., 2000; Kieu et al., 2001; Watson et al., 2007).

Biological tests have the potential to be more environmentally relevant than chemical tests, as they are based on organisms' responses to substances that have toxic effects, and contaminants still present and undetected at low concentrations may have negative effects on biota (Miller et al., 1985; Kettrup \& Marth, 1998; Danilov \& Ekelund, 2001; Roig et al., 2003; Mikol et al., 2007). Giesy and Hoke (1989) suggest that chemical concentration tests are potentially inaccurate with regard to the toxicity to organisms, and bioassays are faster and provide better information about a pollutant's bioavailability. Only a limited number of substances can be tested with chemical analyses, and these do not provide information on toxicity. Further, a large portion of adverse biological effects is caused by unknown substances or a mixture of substances (Durand et al., 2009). Biological responses vary according to the type of contaminants, concentrations of contaminants, exposure time, and bioavailability of the contaminant. For this reason, Cairns \& Mount (1990) emphasize that knowledge of chemical concentration alone is not sufficient to make predictions about the toxicity of a compound. Further, biomonitoring may detect biological effects of contaminants which are not routinely tested for in chemical analyses of drinking water, and it also serves to detect the synergistic or combined effects of contaminants (Winter, 2000; International Joint Commission, 2006; Mikol et al., 2007). Only biomonitoring has the ability to detect the true toxicity of mixtures of substances (Hermens et al., 1984; Giesy \& Hoke, 1989; Fochtman et al., 2000; Durand et al., 2009). Further, thousands of different chemicals are 
present in the water, and measuring the concentration of some will not provide relevant information on the toxic effects of these substances to living organisms (Samoiloff et al., 1983; Miller et al., 1985; Gerhardt et al., 2006b). Chemical testing is also extremely costly, and in some instances biomonitoring may eliminate the need for chemical tests altogether (Miller et al., 1985). A complete biomonitoring system will encompass a minimum number of toxicity tests that will reduce uncertainty and provide predictive information about the toxicity of a substance (Cairns \& Mount, 1990).

The lower cost of maintenance and implementation of a biomonotoring system may also improve the effectiveness of drinking water monitoring programs in general. Most importantly, many biological tests have the ability to produce very fast responses, potentially in less than $24 \mathrm{~h}$. For these reasons biological tests are implemented in early warning systems for water quality assessments, making these more robust and relevant to the environment and therefore to human health as well.

The project to which this study is contributing aims to implement a fully automated biomonitoring system in the Niagara Region in Southern Ontario. Several municipalities supply over 300,000 residents with drinking water taken from Lake Erie via the Welland Canal, including St. Catharines, Thorold, Niagara-on-the-Lake, Lincoln, Welland, Port Colborne, and Pelham (City of Welland, 2008; City of Port Colborne, 2010; City of St. Catharines, 2010). The Welland Canal is a shipping canal and as such is susceptible to contamination from shipping vessels, most notably antifouling agents such as tributyltin (TBT), for which there is evidence that it is still a risk to aquatic organisms despite being strongly regulated since 1989 in Canada (Bartlett et al., 2005). Agriculture is also a very important part of the economy and culture of the Niagara Region (Niagara Region, 2010), and pesticides from agricultural runoff, such as atrazine, used extensively in Canada and especially Ontario (Health Canada, 1993) may enter waterways including the Welland Canal and cause potential contamination for aquatic ecosystems and drinking water users. The region is also a hub of commerce and home to a variety of manufacturing sites including chemicals and chemical products plants and manufactured metal products plants (City of Niagara Falls, 2010). Industrial effluents are considered a risk to the aquatic ecosystems of the region, which includes contamination by heavy 
metals such as copper, which was found in elevated amounts in 2009 in three water quality monitoring stations in the Welland River (NPCA, 2010). The risk of contamination from various sources in the Welland Canal could mean that implementing an automated real-time behavioural analysis system would provide a great advantage for drinking water monitoring in this region.

\subsection{Importance of whole-organism testing}

Whole-organism tests are much simpler and faster than community tests involving species interactions and therefore tend to be cheaper (Newman \& Clements, 2008). Although they will not provide information about how a particular compound will affect interacting species or food webs, whole organism tests can still be environmentally relevant if appropriate test species are chosen (Roig et al., 2003). Ducrot et al. (2005) and Landis and $\mathrm{Yu}$ (1995) have proposed various criteria for selection of test species, including being commonly found and widely available for the researcher, since not only will this affect the ease of the experiment, but this has implications for environmental relevance as well - a test species which is rare will bear less environmental relevance than one that is ubiquitous in the environment and important in the food chain. Organisms should also be easy to culture in the laboratory so that enough quantities are available for the researcher and various repetitions of the experiment. Further, the sensitivity of the organism to a variety of toxicants should also be well defined in the literature, e.g. in aquatic toxicology D. magna is one of the most well-known studied organisms. Other traits which affect the choice of organism are geographic distribution, life cycle, reproductive cycle, habitat preferences, and reproducibility of responses (Ducrot et al., 2005). A high sensitivity to contaminants can give the organism the potential for being an early warning indicator species. However, problems arise when an early warning becomes a false alarm or a false positive; as Forbes et al. (2006) mentions, attempts to distinguish between these two are not often done. 
Whole-organism testing is ideal to determine cause-effect relationships between contaminants and organisms, since variables can be more tightly controlled in the lab (Landis \& Yu, 1995). Further, whole-organism tests are more standardized and have a higher potential for being reproducible, which enables researchers to compare test results more easily, as well as relate them to legally set standards. Some important limitations for whole organism testing still exist, however, when extrapolating one species' results to ecosystem impacts. For this reason many researchers question the value of single-species whole-organism testing (Newman \& Clements, 2008). Forbes et al. (2006) point out that relationships between responses at the individual organism level and the population level are inconsistent, not linear, and only weakly correlated. For example, after analyzing various toxicants' effects on various species, it was found that fecundity, or number of offspring per female, an individual trait, is very weakly correlated with overall population growth rates. Nonetheless, whole-organism responses, especially if looking at sublethal endpoints, have the capacity of producing a response much more rapidly than if looking at community-based endpoints such as community structure, which makes these much more useful as early warning indicators as well as setting standards (Maltby et al., 2002). In fact, single species testing is also necessary in addition to multispecies testing even if the latter is the primary method of testing, since this test is more capable of providing answers with regard to the pathway and mechanism of action of that particular contaminant, and to how it will affect the population or community (Newman \& Clements, 2008).

\subsection{Importance of multiple species testing}

While whole-organism testing provides a balance between environmental relevance and cost, one single species that can provide all relevant information about toxic substances present in the water does not exist. Different species, especially belonging to different taxa and trophic levels, can have dramatically different sensitivities to contaminants and alter toxicity tests (Wenzel et al., 1992; Ducrot et al., 2005). In an aquatic ecosystem, variations in geographic distribution, morphology, physiology, life 
cycles and feeding habits of species will generate different responses and different sensitivities to stressors (Giesy \& Hoke, 1989; Cairns \& Mount, 1990). For a complete collection of toxicity information, the sensitivity of each species present in the aquatic ecosystem would be tested and measured; however, this is not feasible due to the time and cost involved in testing. Further, many of these tests could potentially prove redundant, as some species could be in general less sensitive to all contaminants than others. For this reason, the number of species tested should be minimized, but should represent the overall ecosystem to maximize sensitivity and reliability of responses. There is an overwhelming consensus among researchers to include not only one species when testing for chemical toxicity, but rather a battery of tests which includes a representative variety of taxa and sensitivities (Samoiloff et al., 1983; Miller et al., 1985; Cairns \& Mount, 1990; Clarke et al., 1990; Bitton et al., 1992; Schaeffer, 1994; LAWA, 1996; Wenzel et al., 1997; Adams \& Greeley, 2000; Castillo et al., 2000; Fochtman et al., 2000; Girling et al., 2000; Gullick et al., 2003; Isidori et al., 2003; Roig et al., 2003; Ducrot et al., 2005; Gerhardt et al., 2006b; Zhou et al., 2008). The sensitivity of these organisms must also be balanced by the ability to discriminate and rank the relative toxicity of substances tested (Giesy \& Hoke, 1989). As discussed in section 1.0, the automated real-time biomonitoring system to be implemented in the Niagara Region in Southern Ontario, which this study is contributing to, is being developed to assess endpoints of a variety of species in the aquatic ecosystem, which include plants, algae, and invertebrates.

\subsection{Use of behavioural responses}

There is a variety of possible endpoints when studying the toxicity of a compound at the whole-organism level, which can be either lethal or sublethal. Many ecotoxicological studies use survival/lethality as an endpoint for assessing effects of a contaminant. However, these are generally not very sensitive and may not be ecologically relevant (Giesy \& Hoke, 1989). Sublethal endpoints may provide faster, more sensitive and more relevant responses (Dodson \& Hanazato, 1995; Landis \& Yu, 1995). Behaviour 
is the end result of a long sequence of events involving the stimulation of sensory neurons, neurological responses and release of chemical messages, and a physiological reaction involving motor neurons and muscular contractions (Beitinger, 1990; Kieu et al., 2001; Untersteiner et al., 2003; Ren et al., 2009; Jeon et al., 2008; Gerhardt, 2009). Changes in behaviour are environmentally and ecologically relevant, as these are associated with foraging, reproduction, predation, and escape, all of which can affect the community structure of aquatic ecosystems (Doving, 1991; Fernandez-Casalderrey , 1994; Bunn, 1995; Charoy \& Janssen, 1999). They can also provide essential information about the general fitness and physiological condition of organisms (Baillieul \& Scheunders, 1998; Jeon et al., 2008). The use of locomotory characteristics as endpoints in toxicity tests is consequently ecologically well justified since individual swimming alterations may indirectly account for changes in growth, demographic composition and survival of the population (Charoy \& Janssen, 1999). Behavioural responses are obvious, readily visible, and much faster in comparison to life history trait or mortality studies (Dodson et al., 1995; Ren et al., 2009). Giesy and Hoke (1989) also state that a continuous response variable is preferable to an "all or none" response, which automated behavioural assessments are capable of providing. This study will focus on behavioural responses as indicators of toxic stress in aquatic organisms, in particular locomotory behaviours such as swimming velocity, and more complex ones such as gravitactic and phototactic orientation.

Gravitaxis and phototaxis in aquatic organisms have been extensively studied for over a century and suggested as toxicity endpoints by many researchers (Häder, 1987; Storz \& Paul, 1998; Iseki et al., 2002; Gerhardt et al., 2006a; Martins et al., 2007; Semsari \& Megateli, 2007). In Daphnia magna, for example, phototaxis is an important physiological response which helps the organism orient itself in the water column. This orientation is crucial for predator avoidance during periods of high light intensity, and grazing and foraging during periods of lower light intensity (Cushing, 1951; Gerhardt et al., 2006a). Several studies have indicated that this endpoint is particularly sensitive to contaminants (Storz \& Paul, 1998; Kieu et al., 2001; Martins et al., 2007; Semsari \& Megateli, 2007). In Euglena gracilis, positive phototaxis has been observed under periods of low light, and negative gravitaxis, or photoavoidance, has been observed under periods 
of high light intensity, and is known to be affected by xenobiotics (Iseki et al., 2002). However, gravitaxis has received particular attention as a sensitive toxicity endpoint for this organism after it was discovered that it is not a passive phenomenon, but rather triggered by an active physiological mechanism (Häder, 1987; Lebert et al., 1999; Richter et al., 2001; Streb et al., 2006). The ecological significance, chemical sensitivity, quickness of response, and ease of quantification of these endpoints gives them the potential to be particularly useful for implementation in an early-warning automated biomonitoring system for drinking water.

\subsection{Test organisms}

Five organisms were chosen for this study based on their potential suitability for water quality testing as well as the potential to be used with automated behavioural analysis systems (discussed in section 1.7). This study will evaluate their potential as indicator organisms in the context of an automated "miner's canary" drinking water quality early warning system. As discussed in section 1.0, other species are being tested by other studies in this research project and will not be detailed here. Test species must have known responses to toxic contaminants relevant to drinking water monitoring, be sensitive, widely available, and easy to culture in the laboratory. Their suitability for use in automated systems is based on the sensitivity and reliability of individual parameters tested after exposure to key contaminants.

\subsubsection{Euglena gracilis}

Euglena gracilis (Figure 1.1) is a unicellular photosynthetic freshwater flagellate that belongs to the Euglenacea family, belonging to the kingdom Excavata (previously classified under the now-nonexistent kingdom Protista). It was found to be a suitable organism for implementation in a drinking water early warning system (Tahedl \& Häder, 1999; Girling et al., 2000; Danilov \& Ekelund, 2001; Pearce, 2009). This species can also 
grow heterotrophically in the absence of light. Its taxonomic placement has changed over the years due to the "puzzling" presence of plant and animal cell characteristics; however, it has remained an attractive model organism and has been used extensively in toxicology research because of these characteristics, making it able to assess contaminants that affect both plants and animals. Its ease of handling and genetic stability due to asexual reproduction, in particular with, E. gracilis strain Z, also make this a good model organism (Watanabe et al., 2003; Tahedl \& Häder, 2001). In addition, it is widely distributed in aquatic ecosystems, and is thought to produce fast and predictable responses to a variety of contaminants (Tahedl \& Häder, 1999, 2001; Danilov \& Ekelund, 2001; de Kuhn et al., 2006; Rochetta et al., 2006; Li et al., 2009).

E. gracilis has been recommended in the past as a model organism for growth bioassays, which are considered the most 'classical' toxicity bioassay with this species and algae species in general (Girling et al., 2000; Danilov \& Ekelund, 2001; de Kuhn et al., 2006; Li et al., 2009). In the past, E. gracilis growth was found to be a sensitive parameter to organophosphate pesticides (Moore, 1970) as well as the heavy metals mercury, cadmium, lead (Navarro et al., 1997), nickel (Ahmed \& Häder, 2010b), and chromium (Rochetta et al., 2006). However, acute toxicity tests with E. gracilis have gained greater research interest in recent years. Parameters such as photosynthetic capacity have been measured and found to be sensitive to several contaminants (Danilov \& Ekelund, 2001).

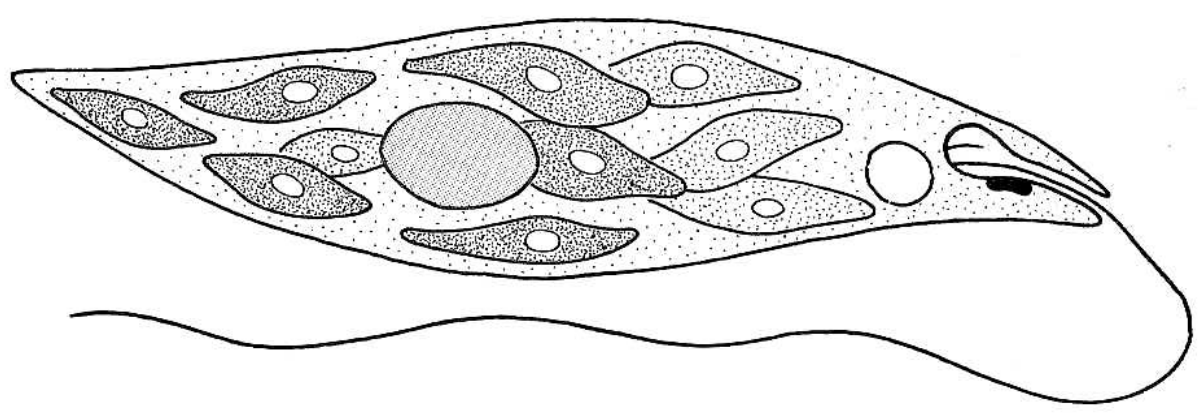

Figure 1.1. Euglena gracilis. ( D.G. Mackean, 2010 
E. gracilis also exhibits several movement behaviours that can be investigated, such as phototactic and gravitactic orientation; that is, they use light and gravity to orient themselves in the water column. Under low light, cells exhibit positive phototaxis, or movement towards the light source, whereas under strong light levels they exhibit negative phototaxis, swimming against the light. In the dark, cells move upwards by negative gravitaxis (Lebert et al., 1999; Richter et al., 2001; Häder et al., 2003; Streb et al., 2006; Richter et al., 2007). Movement and orientation responses are considered very sensitive to toxic substances such as heavy metals and organic compounds (Streb et al., 2002). Iseki et al. (2002) reports that phototactic response in E. gracilis is very sensitive, as cells change swimming direction abruptly with varying blue light intensity. Cells possess a photosensing organelle near the base of the flagellum called the paraflagellar body, which contains flavoproteins as photoreceptor molecules (Iseki et al., 2002). Gravitaxis in particular has been found to be a sensitive endpoint for measuring chemical toxicity (Tahedl \& Häder, 1999; Richter et al., 2001; de Kuhn et al., 2006; Richter et al., 2007). Despite this response having been known for years, only recently has it been investigated in more detail, as it was noted that this behaviour changes with the life cycle of the cells (Lebert \& Häder, 1999; Richter et al., 2007). Because of the lack of a gravitysensing organelle, it was previously thought that negative gravitaxis occurred as a passive buoyancy mechanism where, due to a buoyancy asymmetry, cells rotate with the flagellum facing downwards. However, recent studies have shown that negative gravitactic behaviour in E. gracilis is in fact an active physiological process involving calcium, cAMP, and membrane potential (Lebert \& Häder, 1999; Richter et al., 2001, 2003, 2007; Daiker et al., 2010). Cell reorientation in both gravitactic and phototactic responses is based on cAMP binding to regulating proteins in the flagellum and changing its beating pattern (Lebert \& Häder, 1999; Richter et al., 2003, 2007; Daiker et al., 2010). E. gracilis is also known to change shape under chemical and physical stress conditions, changing from elongated to round (Tahedl \& Häder, 1999; 2001; de Kuhn et al., 2006; Richter et al., 2007), due to a contractile mechanism caused by microtubule depolymerisation in the cytoskeleton (Lachney \& Lonergan, 1985). 


\subsubsection{Daphnia magna}

Daphnia magna (Figure 1.2) is one of the most common species used for singlespecies testing in ecotoxicology. It is the most commonly used cladoceran in ecotoxicology, a field that in the past 10 years has seen cladoceran studies make up almost 10\% of all publications (Sarma \& Nandini, 2006; Ren et al., 2007). This species offers many advantages to biomonitoring, such as its short life span, visible size $(0.5-5 \mathrm{~mm})$, inexpensive costs, and short reproductive cycle (Sancho et al., 2009). They exhibit a higher sensitivity to a variety of contaminants than most

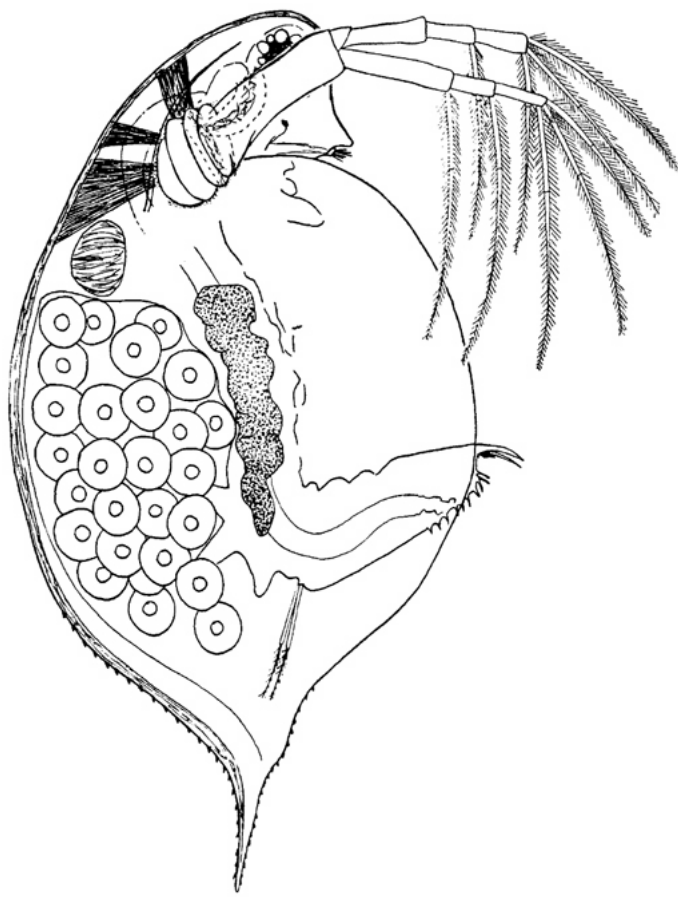

Figure 1.2. Daphnia magna. (C) Tom Little, 2006, University of Edinburgh. vertebrates (Wolf et al., 1998; Untersteiner et al., 2003; Sarma \& Nandini, 2006; Jeon et al., 2008) - so high in fact that it may sometimes become a disadvantage to using this test organism (Landis \& Yu, 1995). Also, many standards exist for comparison (Environment Canada, 1996, 2000; USEPA, 2002; OECD, 2004), making D. magna useful for testing single compounds, mixtures, and effluents. Tests with daphnids and many single-species organisms can be either acute or chronic. Commonly preformed Daphnia tests are the $24 \mathrm{~h}$ and $48 \mathrm{~h}$ acute toxicity tests which have immobility as an endpoint (described as lack of swimming behaviour after prodding), and organisms used are generally neonates less than $24 \mathrm{~h}$ old (Landis \& $\mathrm{Yu}$, 1995; Ren et al., 2009; Shimizu et al., 2002). Previous work by Marshall (2009) has found that $D$. magna behaviour changes rapidly and consistently under concentrations of TBT and atrazine tested, making this organism very suitable for implementation in an early-warning automated biomonitoring system being developed by this research project. The behaviours investigated by Marshall (2009) were swimming height, swimming style, immobilization, secondary antennae use, and spinning, in order of decreasing reliability and sensitivity. 
Behavioural endpoints for D. magna are well studied and provide results in faster time frames than typical $24 \mathrm{~h}$ or $48 \mathrm{~h}$ acute toxicity tests (Wolf et al. 1998; Kieu et al., 2001; Untersteiner et al., 2003; Gerhardt et al., 2006a; Martins et al., 2007; Semsari \& Megateli, 2007; Marshall, 2009). Untersteiner et al. (2003) point out that behavioural parameters for D. magna, especially locomotory behaviour, can provide important information about a contaminant's burden on the ecosystem, since behaviour is "the endpoint of a sequence of neurophysiological events including stimulation of sensory and motor neurons, muscular contractions and release of chemical messages." This would mean that locomotory behaviour would be sensitive to many contaminants with a variety of modes of action. Wolf et al. (1998) also mention that D. magna react to stress situations by diverting energy away from normal metabolic functions to restore the imbalance caused by the environmental stressors, which translate into two separate categories of behavioural reactions: escape (increased swimming) and protection (decreased swimming). This further highlights the usefulness in observing locomotory behaviour as an endpoint for a variety of different reactions from contaminants.

Many studies have observed $D$. magna behaviour in the past given the immense usefulness of this endpoint (Dodson \& Hanazato, 1995; Wolf et al. 1998; Untersteiner et al. 2003; Schmidt et al. 2005; Jeon et al. 2008). Dodson and Hanazato (1995), for example, have found that some xenobiotics can affect vertical migration and spatial orientation, both of which are related to phototactic response of D. magna. Schmidt et al. (2005) also found variations in phototactic response of D. magna, as well as a decrease in swimming velocity after exposure to tributyltin and PCB. Ren et al. (2009) have also observed a decrease in swimming behaviour after the addition of deltamethrin, chlorothalonil and nitrofen. Other studies have found that, beyond a decrease in velocity after exposure to xenobiotics, D. magna also exhibits "disorientation", or erratic swimming behaviour, where individuals spin around at a tight axis. Christensen et al. (2005) observed this behaviour after addition of the neurotoxic insecticide cypermethrin, as did Dodson and Hanazato (1995) after carbaryl exposure, and Shimizu et al. (2002) after copper, organophosphorus, and carbamate exposure (Figure 1.3). 
Various other studies have looked at acute sublethal effects of xenobiotic stressors on D. magna behaviour. Goodrich and Lech (1990) used rather rudimentary methods by today's standards, but still observed a stark effect in vertical migration/phototaxis of $D$. magna after the addition of lindane, more specifically they saw an increase in random migration and a deterioration of phototactic orientation. Sancho et al. (2009) observed a different endpoint which is now gaining more popularity - feeding rates. Their study found that feeding rates decreased after only 5 hours of exposure to the fungicide tebuconazole, making this one of the most sensitive endpoints for observation of the effects of fungicides. This trend continued to be emphasized with Barata et al. (2008), who found that feeding bioassays can be 50 times more sensitive for standardized tests, especially for narcotic chemicals. Feeding bioassays are not a new development and have been carried out for some years, as is seen by Fernandez-Casalderrey et al. (1994) who have demonstrated that feeding rates of Daphnia magna are severely impacted by the addition of endosulfan and diazinon.

(a) Control

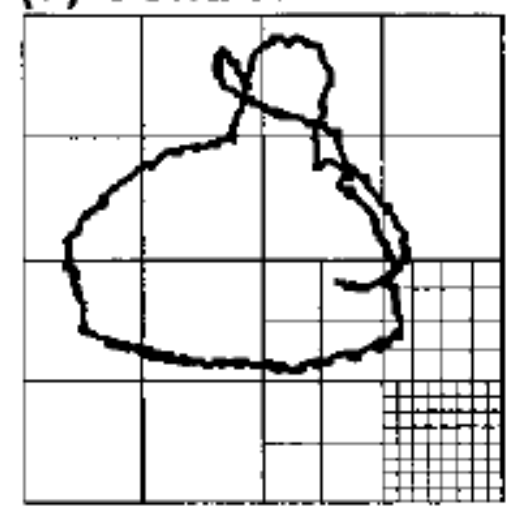

(b) $\mathrm{CuSO}_{4}(10 \mu \mathrm{g} / \mathrm{L})$

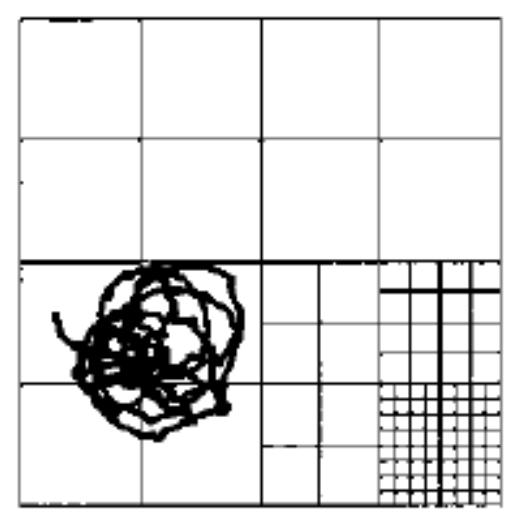

Figure 1.3. Image analysis tracking of swimming trajectory of Daphnia magna, a) under normal conditions and b) under $\mathrm{CuSO}_{4}$ stress. (Shimizu et al., 2002).

With recent developments in detection methods for changes in behaviour, endpoint observation has become more automated, especially with the introduction of 
image analysis systems. Ballieul et al. in 1998 had developed a system for real time image analysis of Daphnid behaviour, and using salinity as a test contaminant, changes in water column position and vertical migration were observed. Similarly, using an image analysis system, Wolf et al. (1998) found that cadmium stress affects D. magna's swimming velocity, swimming participation, and distribution in water column. In more recent developments, Untersteinter et al. (2003) observed behavioural changes in $D$. magna under copper stress using an image analysis system, and found that swimming velocity and swimming participation decrease significantly at higher concentrations of copper and longer exposure times. Image analysis systems have also been able to pick up on the erratic behaviour endpoint, as Shimizu et al. (2002) observed when the addition of $\mathrm{CuSO}_{4}$, organophosphorus, or carbamate caused an increase in "swimming complexity", or erratic swimming, measured as a fractal dimension of the tracked movement (Figure $1.3)$.

\subsubsection{Hyalella azteca}

Hyalella azteca is a benthic amphipod that is used frequently in aquatic toxicology studies due to it being abundant in freshwater ecosystems, being sensitive to a variety of contaminants, its ease of culture in the laboratory, and the high control survival (Nebeker et al., 1984; Blockwell et al., 1999; Bartlett et al., 2005). Additionally, being a benthic organism makes this species a good model organism for exposure to contaminants which may accumulate in sediment, and in fact it is the most used benthic macroinvertebrate in aquatic ecotoxicology (Ingersoll et al., 1998; Hatch \& Burton, 1999; Wang et al., 2004). Despite being used extensively for sediment quality testing, studies have shown that in fact $H$. azteca is more predominantly exposed to contaminants through the water and food sources more than sediment (Wang et al., 2004; Moore et al., 2006). Protocols for survival and growth tests using $H$. azteca have been held by Environment Canada since 1997 (Environment Canada, 1997). This organism is easy to culture and maintain in the laboratory (Wang et al., 2004), and is the most common benthic amphipod in North America and particularly abundant in the Great Lakes region (Borgmann et al., 1996; Hatch \& Burton, 1999). 
Under control conditions, this organism is found in the top layers of sediment close to the sediment-water interface and moves along the sediment by crawling and swimming (Wang et al., 2004). Under stress conditions H. azteca burrows deeper into the sediment but emerges after a period of time, and this is considered an avoidance behaviour to both physical and chemical stress (Hatch \& Burton, 1999; Wang et al., 2004). H. azteca has been found to be sensitive to a variety of contaminants such as copper, zinc, lead and mercury (Borgmann et al., 1996), and particularly to TBT (Bartlett et al., 2005; Marshall, 2009), which will be used in this study. Bartlett et al. (2005) found that $H$. azteca is an ideal species to use in TBT bioassays because it equilibrates rapidly with environmental concentrations of TBT, chronic toxicity with this contaminant has been observed and measured, and it can be used to predict toxicity which is equivalent to four other aquatic invertebrates.

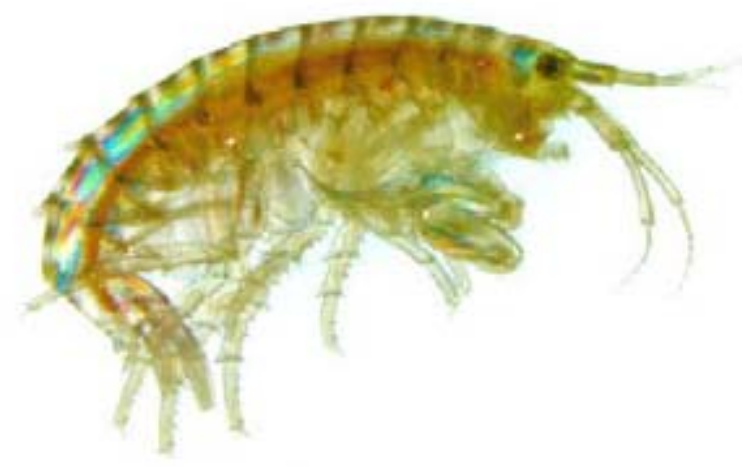

Figure 1.4. Hyalella azteca. (c) U.S. Geological Survey.

More recently, sublethal responses have been examined in $H$. azteca, including behavioural bioassays such as precopulatory behaviour (Blockwell et al., 1998), feeding rates (Gillespie Jr. et al., 1997; Ingersoll et al., 1998), burrowing and grouping behaviour (Hatch \& Burton, 1999; Wang et al., 2004). Changes in movement behaviours of $H$. 
azteca have been previously noted and determined to be a useful parameter to add to an early warning system for drinking water (Marshall, 2009). Given the facts that this species is susceptible to contaminant exposure through water, has found to be sensitive to contaminants, and shows easily observable behavioural changes under stress, this species will be included in this study to investigate its possible use with the automated image analysis system DaphniaTox, discussed in section 1.7.4, for implementation in an automated early warning system for drinking water.

\subsubsection{Brachionus spp.}

Brachionus calyciflorus is a common freshwater rotifer that has been extensively studied in aquatic ecotoxicology due to its ubiquitous distribution globally, simple life cycle (reproducing asexually through parthenogenesis under ideal conditions, much like D. magna), and short generation time (Snell \& Janssen 1995; Huang et al., 2007). Further, they play a key role in freshwater ecosystem and can severely affect algal composition and water quality (Snell \& Janssen 1995). They are an important part of the microbial loop, a pathway of carbon and nutrient cycling in freshwater ecosystems, where there is a fast recycling of organic carbon, which stays in the biomass and is made available to higher trophic levels (Wetzel, 2001). Shifts in rotifer density may for this reason drastically affect community assemblage throughout the aquatic food web.

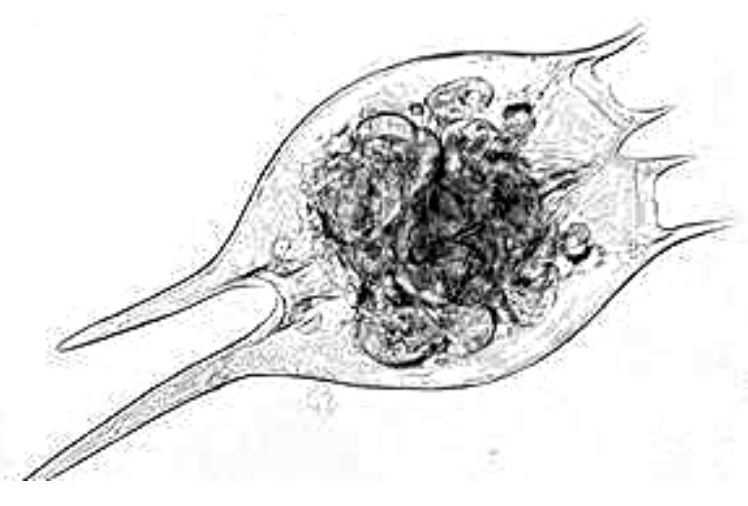


Figure 1.5. Brachionus havanensis, Fussman lab, McGill University.

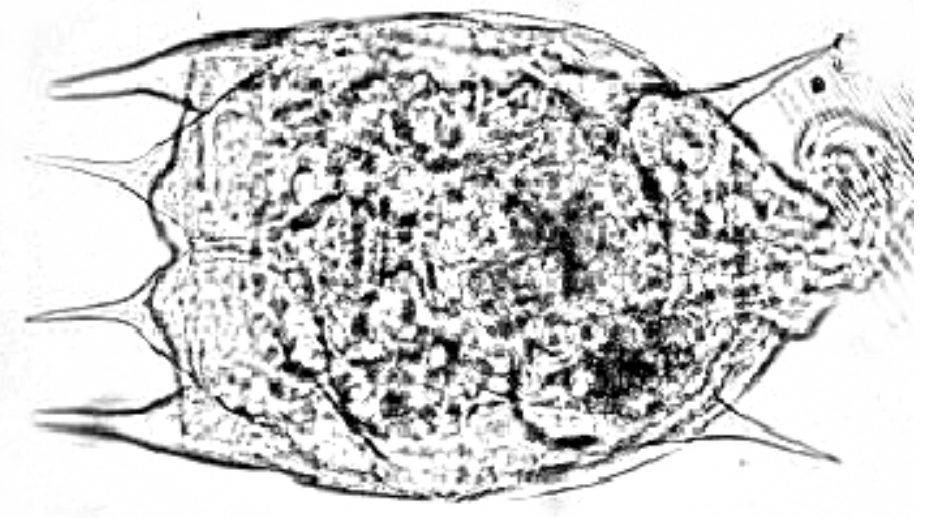

Figure 1.6. Brachionus calyciflorus. Durand et al., 2009.

Although growth bioassays are common with B. calyciflorus, protocols exist for acute 24h mortality since 1991 through the American Society of Testing and Materials (ASTM). 24h mortality test kits are commercially available (Rotoxkit $\mathrm{F}^{\mathrm{TM}}$ ) and are sensitive to a variety of contaminants (Isidori et al., 2003; Thomas et al., 2009). Rotifers have also been previously shown to be more sensitive to some contaminants than cladocerans such as D. magna (Isidori et al., 2003; Snell \& Joaquim-Justo, 2007). If included in the automated early-warning system in development, there is the potential to make the system overall more sensitive but also more able to discriminate and identify contaminants since it may be more sensitive to some contaminants but less sensitive to others. Changes in swimming behaviour of Brachionus calyciflorus have been measured with automated image analysis systems in the past, where it was found to be sensitive to copper, PCP, and lindane after only $2 \mathrm{~h}$ in concentrations as low or lower than the $24 \mathrm{~h}$ LC $_{50}$ (Charoy \& Janssen, 1999). In Brachionus spp., swimming is performed by the coordinated beat of the cingulum cilia, and is controlled by two innerved muscles. Modifications in the swimming characteristics are a result from changes of the cilia 
beating ability (Charoy \& Janssen, 1999). Swimming behaviour in rotifers, as in other freshwater species, has been shown to play an important role in several ecological processes such as mating behaviour, foraging activity, and predator avoidance, and these toxicity endpoints are therefore ecologically relevant as they can alter community composition (Charoy \& Janssen, 1999; Snell \& Joaquim-Justo, 2007).

Brachionus spp. will be investigated in this study as potential species to be implemented in an early warning system for drinking water in conjunction with the automated system ECOTOX. Using multiple species with one automated system will make the overall early-warning system more efficient, less costly, and requiring less maintenance and manpower overall (Gerhardt et al., 2006b). B. calyciflorus was chosen for being a common bioassay organism, and B. havanensis was chosen for its likely equivalent sensitivity to contaminants as B. calyciflorus, and for its smaller size, at 200$250 \mu \mathrm{M}$ as opposed to B. calyciflorus's 250-300 $\mu \mathrm{M}$ (A. Derry, personal communication, November 2009); this has the potential to make the former more easily usable with the small, microorganism-adapted chambers of the ECOTOX image analysis system (discussed in section 1.7.3).

\subsection{Automated Biomonitoring Systems}

According to Newman and Clements (2008), improvements in biomonitoring require innovative techniques that will improve efficiency without sacrificing the relevance and quality of data being produced. Technological innovations in ecotoxicology testing are now allowing researchers to look into a wider variety of endpoints that can potentially be incorporated into early warning biomonitoring systems, which can detect contamination of drinking water before it is released into the drinking water supply. Flow-through online monitoring, for example, can be used not only to detect responses much faster than standard $24 \mathrm{~h}$ acute toxicity tests, but it also further allows continuous monitoring of effluents (Lebert \& Häder, 1999; Tahedl \& Häder, 1999, 2001; Gerhardt et al., 2006b; Lechelt et al., 2006). Much information can be gained by developing toxicity models based on acute, sublethal responses of organisms to 
xenobiotic stressors, especially in the assessment of drinking water quality, as this field requires fast and accurate responses which cannot be provided by chemical analysis at all (Samoiloff, 1989; Gerhardt, 2009).

In the past, toxicity tests which were acute were commonly associated with lethality as an endpoint, whereas chronic tests, which usually last a portion of the organisms life cycle, were associated with sublethal endpoints (Giesy \& Hoke, 1989; Tahedl \& Häder, 2000). Image analysis systems now have the potential to allow researchers to easily look at sublethal endpoints in acute toxicity tests in a way that is automated and requires minimal supervision. Especially in the last twenty years, new technologies started to allow researchers to look at sublethal endpoints at the organism level more efficiently. Advances in image analysis and other detection mechanisms, coupled with flow-through and online systems, enable the capture of results in real-time. This continuous monitoring of behavioural responses can make these devices particularly useful for early-warning biomonitoring, which is ideal for drinking water quality monitoring or industrial waste water (LAWA, 1996; Roig et al., 2003; Untersteiner et al., 2003; Gerhardt et al., 2006b; Durand et al., 2009). The most common test species include fish, bivalves, Daphnia spp., algae, and bacteria. These early warning systems include both image analysis and non-optical systems.

\subsubsection{Non-optical systems}

Non-behaviour automated biomonitoring tests using microorganisms commonly included in a battery of tests for toxicity assessment are the Microtox ${ }^{\circledR}$ and the PAM fluorometer test (Durand et al., 2009). The PAM fluorometer looks at photosynthetic activity of Pseudokirchneriella subcapitata by measuring chlorophyll fluorescence, whereas the Microtox ${ }^{\circledR}$ system, commercially available since the early 1980 s, looks at bioluminescence of Vibrio fischeri, also known as Photobacterium phosphoreum, a marine bacterium (Schaeffer, 1994; Durand et al., 2009). The Microtox ${ }^{\circledR}$ system's major weakness is that the bioindicator species used is not environmentally relevant in the context of a freshwater system. Problems also include biofilm formation (LAWA, 1996), 
continuous growth of the bacteria, low sensitivity, and addition of nutrients and media manipulating the test water characteristics (Gerhardt et al., 2006b), and the fact that this system is lab-based only and cannot be implemented in-situ (Roig et al., 2003).

Common non-optical behaviour analysis systems commercially available today include the MosselMonitor ${ }^{\circledR}$, which can be used with a variety of both marine and freshwater bivalve species, such as Dreissena polymorpha, Unio pictorum, or Mytilus edulis. Rather than using images to collect data, the MosselMonitor ${ }^{\circledR}$ measures the distance between shell halves by fitting a miniature coil on each, creating a magnetic field that can be measured (Roig et al., 2003). A signal is sent through the first coil, which induces a voltage in the second coil, and the strength of this voltage is a measure of distance between shell halves (Roig et al., 2003). The Dreissena Monitor also uses Dreissena bivalves, operates in a similar manner, and is commercially available and extensively used in several water monitoring stations in Germany (LAWA, 1996). The main disadvantages of using bivalves are a high mortality rate and the fact that they cannot be grown in the laboratory and must be collected from the wild (LAWA, 1996). Another example of a non-optical system is the Multispecies Freshwater Biomonitor ${ }^{\mathbb{B}}$, which uses electrodes to measure changes in the current caused by movements of the organism (Gerhardt, 2007) and is being investigated by V. Fleet as part of the overall project for development of an automated early-warning system or monitoring drinking water quality in Southern Ontario.

\subsubsection{Image analysis systems}

BehavioQuant $\AA$, also known as the Koblenz Behavioral Fish Test, commonly used with the freshwater species Leuciscus idus, is one example of an image analysis system commercially available today and in use at the German Federal Institute of Hydrology (Butterworth et al., 2001). This particular system analyzes fish behaviour to determine various parameters such as swimming speed, number of turns, and distances between individuals, as seven individuals are kept per chamber in this system (Staaks \& Baganz, 2006). Other fish biomonitoring systems exist, with some differences. Some are 
made to observe parameters of one individual only rather than interactions between individuals, and will therefore have only one individual per chamber, such as the USACEHR Fish BioMonitoring System, which measures ventilatory rate, cough frequency, and body movement patterns (Roig et al., 2003). Another difference with this system is that it was made to be used with a specific fish species, the bluegill Lepomis macrochirus. Such is also the case with the BBE Moldaenke Fish Toximeter, another fish behaviour image analysis system, which uses zebrafish (Danio rerio) specifically, although other species can also be employed. BehavioQuant ${ }^{\circledR}$, on the other hand, was made to be used with a variety of species, with D. rerio and sunbleak (Leucaspius delineatus) having been used most extensively (Staaks \& Baganz, 2006). Using fish as bioindicator organisms have been found to be a disadvantage due to low chemical sensitivity as well as generating ethical concerns (Roig et al., 2003; Gerhardt et al. 2006b)

Image analysis systems are also commonly used with D. magna, such as the Dynamic Daphnia Test and the bbe Daphnia Toximeter. The Dynamic Daphnia Test, measuring activity and swimming behaviour via infrared sensors, was developed much earlier than the Daphnia Toximeter. The latter was based on the Extended Dynamic Daphnia test and measures many more parameters such as water column height, distance between individuals, and fractal dimension, or randomness of swimming patterns (Lechelt et al., 2000). Its structure is a flow-through system containing a chamber for $D$. magna which is attached to an optical camera and a computer-based analyzer to process the images (Figure 1.7).

Automated behavioural image analysis systems are being continually developed and improved. One such new system is the Petrel Swimming Behavior Spectrometer (SBS), which should become commercially available in 2011 (Flagship Ventures, 2009). It is based on image analysis of swimming patterns of ciliated protozoa, and preliminary tests have found it to be sensitive to a variety of contaminants including heavy metals, organophosphates, and cyanides. The ciliates are pumped into an observation chamber where they are incubated with the sample solution for 1-6 minutes, and software tracks their responses, triggering an alarm system if behaviour deviates from the norm. The cost 
is estimated to be US $23 \notin$ per assay, and its parent company claims only $1 \mathrm{~h}$ of labour is required for every month of continuous testing, which would make it very competitive in the market (Flagship Ventures, 2009). One of the systems being investigated in this study, ECOTOX, works on a very similar principle to the SBS, where it analyzes the movement of flagellates rather than ciliates.

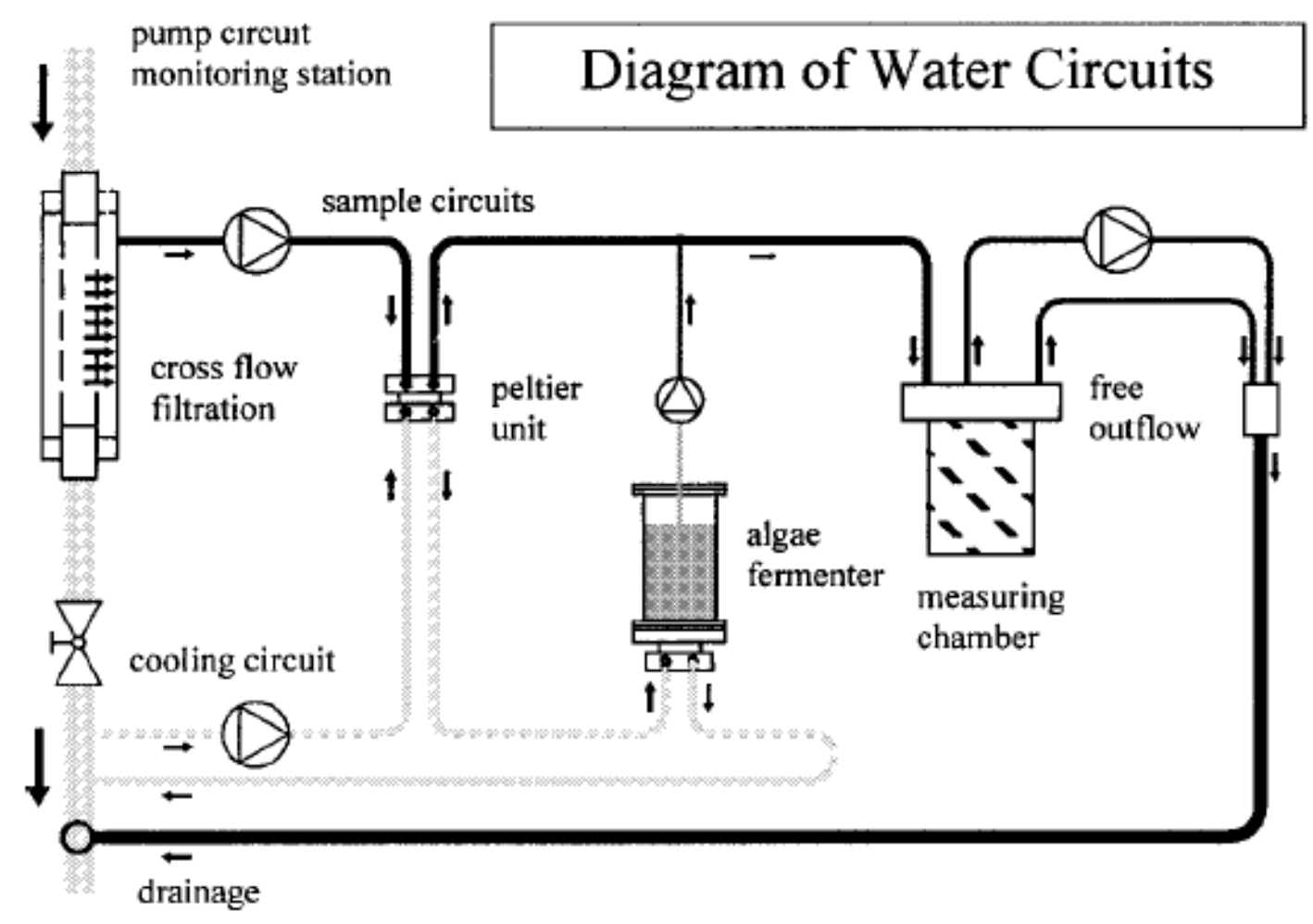

Figure 1.7. Diagram of German-made bbe Daphnia Toximeter demonstrating the flow-though pathway of test water.

\subsubsection{ECOTOX image analysis system}

ECOTOX is one of many image analysis systems in existence today which are being used to provide information on the toxicity of compounds. It is an automatic realtime behaviour analysis system that tracks several movement parameters of the flagellate Euglena gracilis. It is based on the tradition of using single species bioassays to 
determine the effects of pollutants on these organisms and extrapolating this information to infer the effects of this substance on the environment (Tahedl \& Häder, 2000). Test organisms will produce a response which is dependent on the dose, which is in turn dependent on the level of exposure of said organism to the substance being tested. The exposure is equivalent to environmental concentrations of a compound; for aquatic organisms this would be the concentration of a compound in the surrounding waters. The dose of the compound to actually affect the organism in question will depend on various internal factors such as size, surface-to-volume ratio, fat content and type of surface covering of the organism, as well as external factors such as temperature, $\mathrm{pH}$, and hydrophobicity of the compound (Landis \& Yu, 1995).

ECOTOX differs from other image analysis systems such as BehavioQuant and Dynamic Daphnia test in that these are flow-through systems and ECOTOX is not. For example, automated fish tests are carried out as fish swim against the flow-through stream and the number of impulses generated by its muscle movement is quantified. ECOTOX, on the other hand, is not made up of flow-through chambers, but instead carries out short-term static tests within the observation cuvette (Tahedl \& Häder, 2000), which is placed vertically to allow for observation of gravitactic behaviour (Streb et al., 2002) (Figure 1.8). However, this system is still considered a continuous monitoring system, as it can be used to take water samples being tested from a flow-through system at set time intervals. The main behavioural parameters measured by ECOTOX are: 1) motility, given in percentage of motile cells (number of moving cells in proportion to the total number of moving and non-moving cells); 2) swimming velocity, taken as the average of all motile cells in $\mu \mathrm{m} / \mathrm{s}$, or upwards, downwards, or sideward motile cells; 3 ) orientation, given as a direction histogram, percentage of upwardly mobile cells, and an rvalue, which determines the precision of orientation (Appendix A); and finally 4) the shape of the cells, given as compactness, or a form factor which is the ratio of circumference to area of the cell (ECOTOX Version 5.0 manual). All of these parameters are observed in parallel in on-line mode, or real time, and thresholds can be predefined by the researcher so that deviations exceeding these values will trigger an alarm (Streb et al., 2002). In addition to cell monitoring, physical parameters can also be observed, including temperature, $\mathrm{pH}$, and oxygen level. 


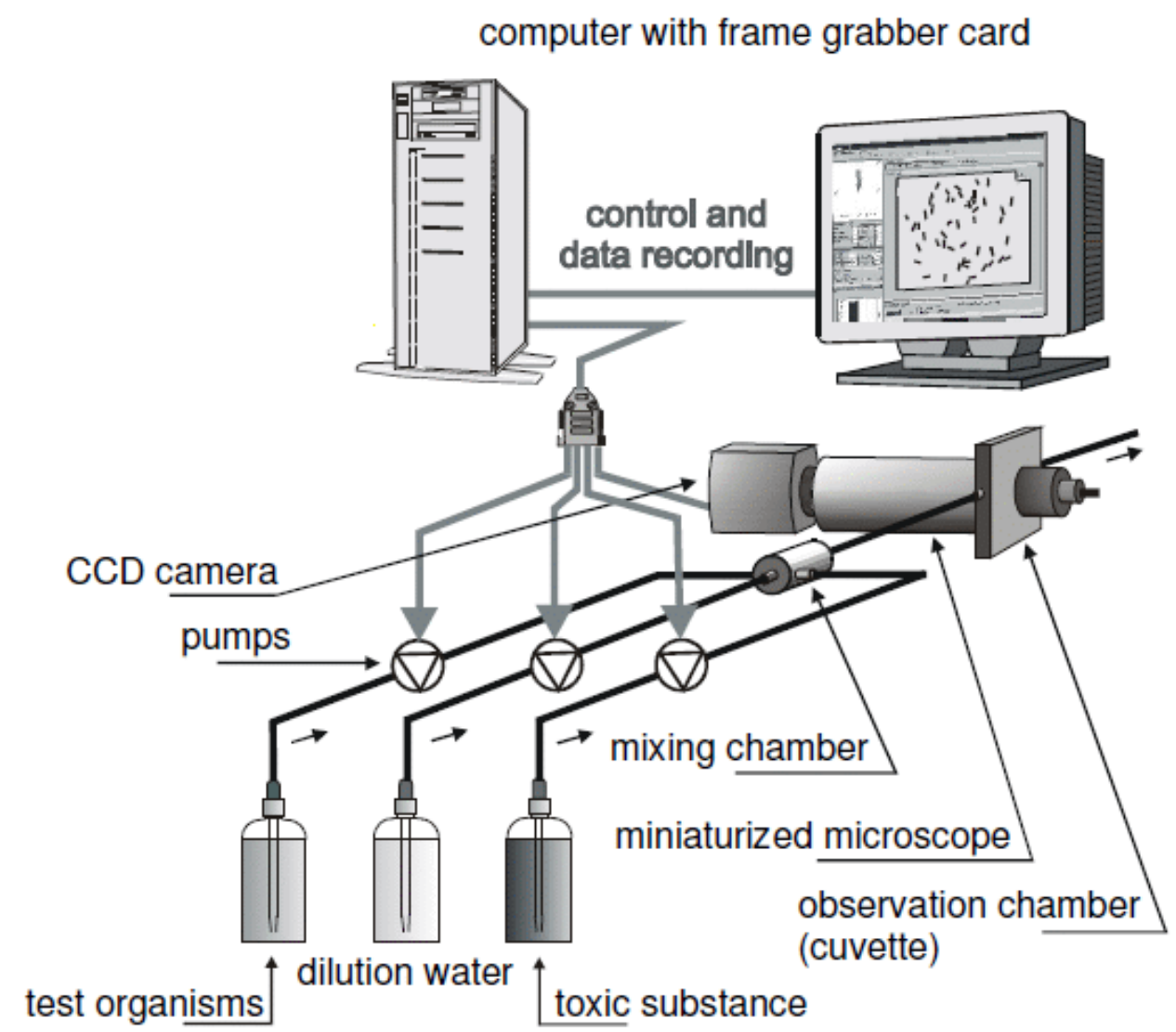

Figure 1.8. Schematic diagram of the ECOTOX Image Analysis System (ECOTOX 5.0)

One of the most important parameters measured by ECOTOX is the gravitactic orientation of cells. The vectors determined by tracking are used to determine the percentage of cells moving upwards, and it also determines the r-value, which is a value for the precision of orientation. The r-value, determined by the Rayleigh test, ranges from 0 to 1,0 being completely random movement in no particular direction, and 1 being all cells moving in the same direction (Tahedl \& Häder, 1999). The software can also create circular histograms which show the proportion of cells moving in a particular direction, set at $15^{\circ}$ intervals, a standard which can also be changed by the user (Tahedl \& Häder, 1999). In the circular histograms, upward swimming cells, or negative gravitactic movement seen under normal conditions, are represented in the $0^{\circ} / 360^{\circ}$ direction, while downward movement is represented by $180^{\circ}$ (Figure 1.9). Other parameters relating to 
orientation include an alignment value, used in conjunction with the r-value, as well as the angle of the main distribution, $\Phi$, towards which the majority of cells are moving (ECOTOX Ver. 5.0 manual). The vectors calculated by image analysis are also used to determine percentage motility, mean velocity of motile objects and compactness, which is determined by the ratio of circumference to area of the cell.

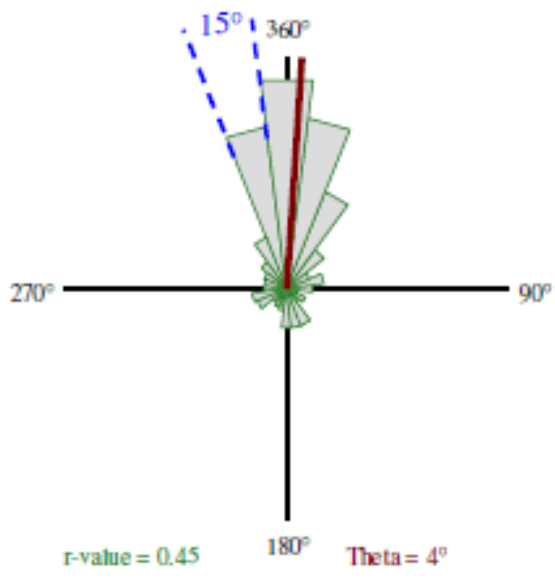

Figure 1.9. Example of a circular histogram showing Theta, the segments representing a range of swimming angle (which can be adjusted by the user through the software), and the r-value based on uniformity of swimming direction. (ECOTOX 5.0 Manual)

Previous studies have found that ECOTOX parameters examining E. gracilis behaviour exhibit sensitivity to a variety of compounds. Under heavy metal stress such as copper, cadmium, and mercury, it shows impaired motility and gravitactic orientation, while the addition of 2,4-Dichlorphenol, a herbicide intermediate, caused decreased motility, velocity, and compactness (Streb et al., 2006). Tahedl and Häder (1999) using found that sensitive endpoints for E. gracilis for formaldehyde toxicity were the r-value and speed. The r-value is closer to 1 for cells under normal conditions (that is, they are swimming in a more uniform direction), whereas under stress conditions the r-value approaches 0 as cells start to swim more randomly (Figure 1.10). The sensitivity was determined by comparing the $\mathrm{EC}_{50}$ for different parameters. For example, this test found that the $\mathrm{EC}_{50}$ for formaldehyde was $0.003 \%(\mathrm{~V} / \mathrm{V})$ for speed and $0.0025 \%(\mathrm{~V} / \mathrm{V})$ for $\mathrm{r}$ - 
value (Figure 1.11). The parameters measured by ECOTOX were found to have different sensitivities for different contaminants, which raises the possibility of determining a category of responses for different contaminants. In 1999, Tahedl and Häder found that gravitactic orientation showed sensitive $\mathrm{EC}_{50} \mathrm{~S}$ for ethanol and cycloxydim, a herbicide. However, heavy metals such as mercury and copper had a lower $\mathrm{EC}_{50}$ for speed rather than orientation. In 2001, the next study published by Tahedl and Häder contained a table of which parameters had the lower $\mathrm{EC}_{50}$ for a range of contaminants. The following compounds had r-value as their most sensitive parameter: PCP, 2,4-DCP, formaldehyde, $\mathrm{Ag}^{+}$, and $\mathrm{Cr}(\mathrm{VI})$. The compounds that had velocity or motility as their sensitive endpoint were TBBPA, $\mathrm{Ni}^{2+}, \mathrm{Pb}^{+}$and $\mathrm{Zn}^{+}$. Many other compounds had compactness as their sensitive endpoint: acetone, $\mathrm{As}(\mathrm{III}), \mathrm{Cu}^{2+}$, and $\mathrm{CN}^{-}$.

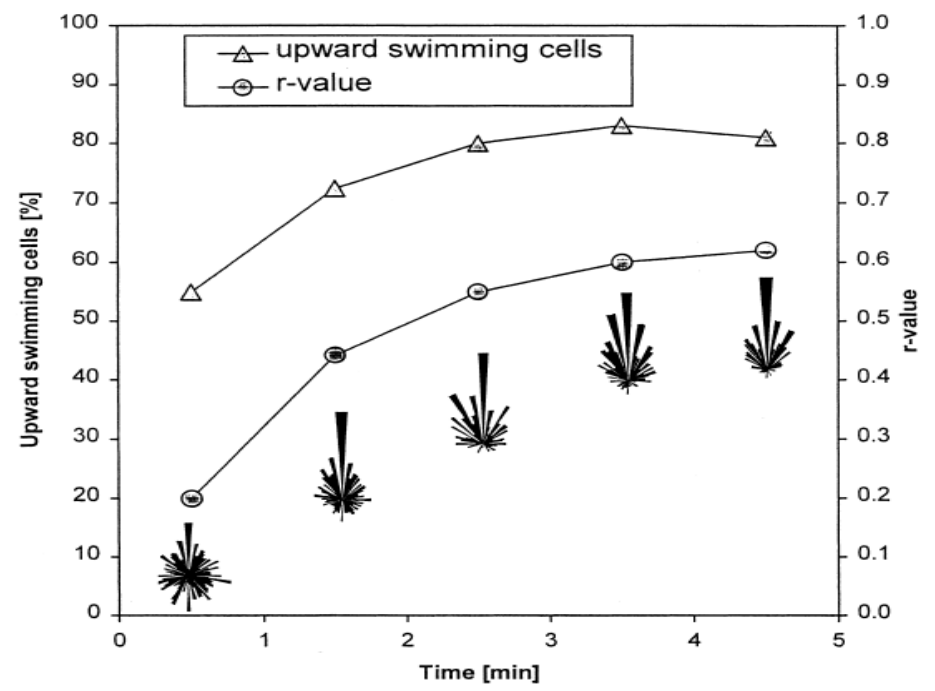

Figure 1.10. Percentage of upwards-swimming cells, r-values, and circular histograms of Euglena gracilis behaviour in the absence of contaminants within the first 5 minutes of entering the ECOTOX chamber. As cells adapt, swimming direction becomes more uniform (Tahedl \& Häder, 1999).

Other studies have used ECOTOX in a variety of ways. Streb et al. (2006) used ECOTOX to determine whether Euglena gracilis could be used as a model system for metabolite toxicity, since previous studies had compared this species with mammalian 
hepatocytes, which are liver cells involved in metabolizing drugs. They also used ECOTOX to identify solvent effects used with the samples, and found that, while $2 \%$ DMSO had clear inhibitory effects, a $1 \%$ concentration produced effects for only a few minutes and was deemed acceptable. However, $\mathrm{KOH}$ was also used as a solvent with (s)warfarin and chlorzoxazone, since even with $1.7 \mathrm{mM} \mathrm{KOH}$ none of the parameters were affected. By looking at longer incubation periods with contaminants, this study found that $E$. gracilis has the ability to metabolize contaminants, indicating that longer exposure times can create metabolites (such as 7-hydroxywarfarin from (s)-warfarin) and therefore longer exposure tests can provide more information on the toxicity of compounds and metabolites. In another study, de Kuhn et al. (2006) used ECOTOX to test various marine flagellates for sensitivity as a criterion for suitability of use for sea water quality bioassays. Using copper as a model contaminant, the study found that Prorocentrum minimum had the highest sensitivity and could successfully be used with ECOTOX. These studies show that ECOTOX can be used in a wide variety of ways.
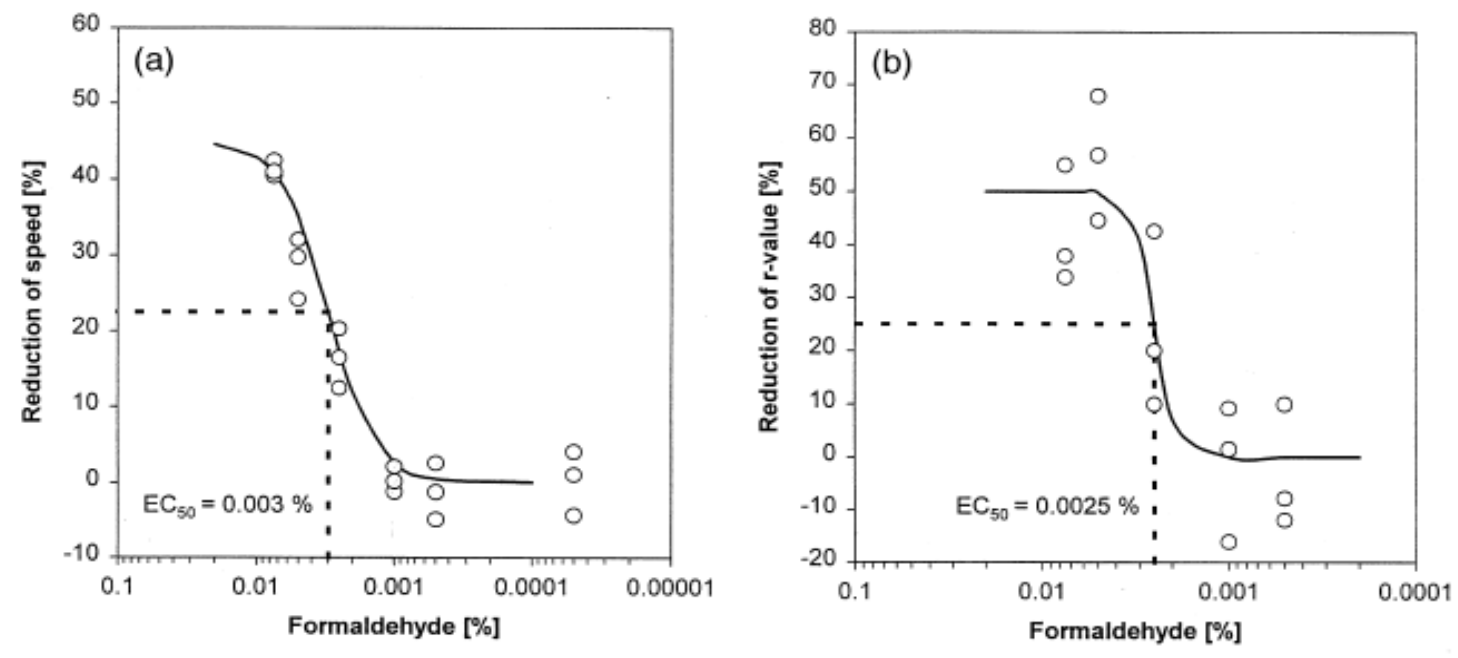

Figure 1.11. Dose-response curves built using the ECOTOX image analysis system, showing that $r$-value is a more sensitive endpoint than swimming velocity to the presence of formaldehyde (Tahedl \& Häder, 1999). 


\subsubsection{DaphniaTox image analysis system}

DaphniaTox works based on the same image analysis principles used by ECOTOX, as discussed in section 1.7.3, but which analyzes the locomotory behaviour of D. magna, and has the potential to look at other macroinvertebrate species such as $H$. azteca as well. This makes it a good potential addition to the early-warning drinking water monitoring system being developed, as it would reduce the amount of personnel training and therefore the overall cost of the system, and incorporate organisms which have been previously shown to be a good addition to the system. It is not a widely used system; however, the principles of movement measured are equivalent to the ones measured by bbe Daphnia Toximeter, with a few differences. The DaphniaTox (Figure 1.12 ) is equipped with a blue LED light for measuring phototactic responses of $D$. magna, whereas the bbe Daphnia Toximeter does not measure this parameter. Instead, it measures other swimming characteristics of D. magna such as grouping and "fractal dimension".

DaphniaTox consists of a hardware and a software component. All the hardware is contained in a small case measuring $29 \times 20 \times 11 \mathrm{~cm}$ and consists of a camera pointed at a platform where one or two cuvettes can be placed, in this case they are disposable, commercially available polystyrene canted neck tissue culture flasks. Red LED lights are placed at the four corners of the side facing the camera, hidden by an adjustable panel to reduce glare on the camera and flask surfaces. Immediately above the flasks are the blue LED lights for activation of phototactic response. The software movement tracking uses vectors in the exact same way as ECOTOX, and produces similar ".ect" files containing swmming velocity, upwards velocity, r-value, and percent individuals swimming up (discussed in section 1.7.3), which can be opened using Microsoft Excel. The software tracks D. magna for one minute at set intervals, e.g. 10 minutes, and recordings continue until they are stopped manually. When the phototactic response setting is selected, the system records organisms for an additional minute with the blue phototactic LED light on, and produces a separate ".ect" file with readings. As the name implies, DaphniaTox was developed for use with D. magna, but this study will investigate its use with an additional invertebrate, $H$. azteca, as well. 


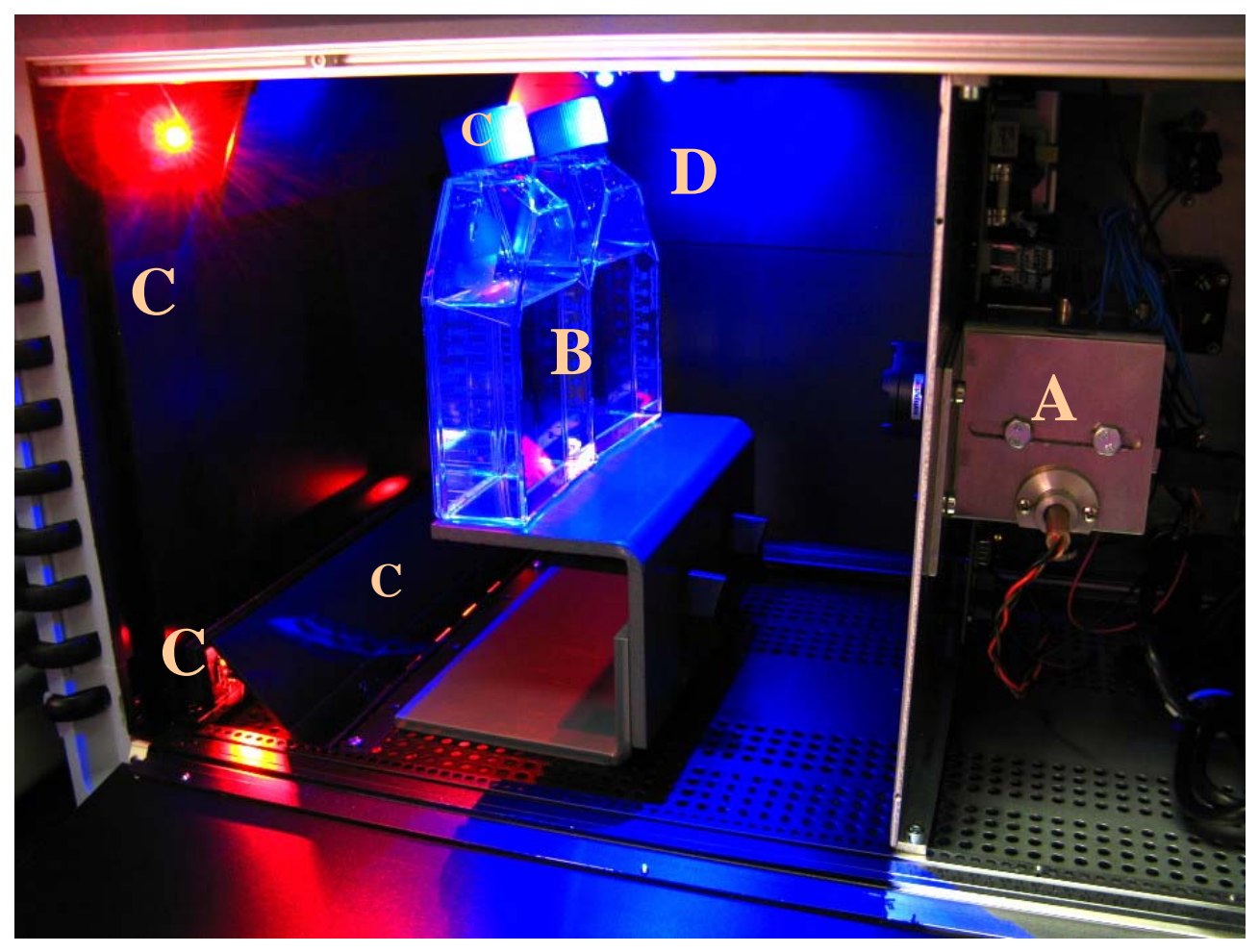

Figure 1.12. The DaphniaTox image analysis system, showing $A$ ) the camera attached to a firewire cable; B) two observation flasks, C) red LED light sources; and D) blue phototactic LED light sources.

\subsection{Contaminants}

The contaminants chosen for this study are contaminants of concern that have known toxicity to aquatic invertebrates and for which maximum concentration guidelines exist in Ontario. The three contaminants chosen represent different categories of contaminants that may act as the start of a database of responses for the organisms and automated systems tested. Contaminants have also been tested repeatedly in other studies contributing to the overall project for development of an early-warning drinking water quality monitoring system for the purpose of sensitivity comparison between organisms and toxicity endpoints. Atrazine and TBT have been tested by Marshall (2009) and Pearce (2009), and currently V. Fleet as well. Copper has been tested by Dort (2010). 


\subsubsection{Atrazine}

Atrazine, or $\mathrm{C}_{8} \mathrm{H}_{14} \mathrm{ClN}_{5}$, is a persistent organic triazine herbicide widely used worldwide in a variety of crops such as maize, corn, soybean, sugar cane, sorghum, and others, to control broadleaf and grassy weeds (World Health Organization, 2003; Lockert et al., 2006; Lazorko-Connon \& Achari, 2009). It is highly persistent having a half-life of 12 weeks to 2 years in aquatic ecosystems depending on $\mathrm{pH}$ (Health Canada, 1993). Its $\mathrm{K}_{\text {ow }}$ is 2.3 and its solubility in water is $30 \mathrm{mg} / \mathrm{L}$ at $20^{\circ} \mathrm{C}$ (IARC, 1999; World Health Organization, 2003), making it likely to sorb to soil and sediment particles (Health Canada, 1993; Graymore et al., 2001). It reaches aquatic ecosystems mainly through storm runoff and spring runoff (Health Canada, 1993; It is considered a priority substance by the USEPA and the European commission for its potential to contaminate groundwater and surface water (Lazorko-Connon \& Achari, 2009).

Concentrations of atrazine as high as $691 \mu \mathrm{g} / \mathrm{L}$ have been found in surface waters in the past after storm events (Lockert et al., 2006), and as high as $1000 \mu \mathrm{g} / \mathrm{L}$ in rivers next to atrazine-applied land (Denoyelles et al., 1982). Its use in Canada began in the 1950s (Detenbeck et al., 1996; Anderson \& Zhu, 2004), although it is being phased out for most uses in this country except for corn crops, where it is used for 1.1 million hectares, or 94\% of Canada's corn production, in Ontario and Quebec. The use of atrazine is not permitted in British Columbia or the European Union, although it continues to be the second most widely used pesticide in the United States (LazorkoConnon \& Achari, 2009) and the most heavily used pesticide in North America overall (Graymore et al., 2001). An accidental atrazine discharge in the Rhine River, detected by the Dynamic Daphnia Test automated biomonitoring system was in fact what triggered the widespread use of biological early warning systems in Europe in the 1980s (Gerhardt et al., 2006b). The Canadian guidelines for atrazine residues in drinking water is $5 \mu \mathrm{g} / \mathrm{L}$ (Health Canada, 2007), although the USEPA and WHO recommend that drinking water contain no more than $2 \mu \mathrm{g} / \mathrm{L}$ based on findings from several studies (World Health Organization, 2003). 
Atrazine is commonly found in drinking water, at concentrations of up to $5 \mu \mathrm{g} / \mathrm{L}$ (World Health Organization, 2003). Humans are exposed to atrazine through consumption of contaminated drinking water, which has been linked to a number of acute and chronic health issues such as nausea and reproductive system tumors, and it is therefore classified as a possible carcinogen to humans (Group 3) (Health Canada, 1993). It was also found to be an endocrine disruptor (Lazorko-Connon \& Achari, 2009). Aquatic organisms have been found to be affected by atrazine exposure as well. It was found to reduce hatching success and cause abnormal larvae development in Chironomus tentans at $230 \mu \mathrm{g} / \mathrm{L}$, and found to change swimming behaviour and motility of zebrafish at $6 \mu \mathrm{g} / \mathrm{L}$ (Dewey, 1986).

Atrazine will be used in tests with the automated image analysis systems as representative of the organic herbicide class of contaminants.

\subsubsection{Tributyltin}

Tributyltin, or TBT, $\mathrm{C}_{24} \mathrm{H}_{54} \mathrm{OSn}_{2}$, is an organometal that has been extensively used in the past as an antifouling agent on boats, ships, and harbour platforms and structures (Choi et al., 2010). It is highly persistent in the environment, having a low solubility in water, $71.2 \mathrm{mg} / 1$ at $20^{\circ} \mathrm{C}$, and a $\mathrm{K}_{\text {ow }}$ of 3.19-3.84 (United Nations Environment Programme, 2006). It has the ability to bioaccumulate, and in aquatic environments they are expected to mostly partition to the sediment. The major source of TBT to the aquatic environment is from leaching of antifouling paints from ship hulls (Borgmann et al., 1996; Alzieu, 1998; Konstantinou \& Albanis, 2004). Antifouling uses of TBT began in the 1960s (Chau et al., 1997). Solvents are often included in ship paints to facilitate application, which makes these more soluble in water (Alzieu, 1998). The Government of Canada's future management strategy for TBT is virtual elimination. Its use as an antifouling agent in ship hulls has been controlled in Canada since 1989 and the sale of TBT-containing antifouling paints has been prohibited since 2003, though it is estimated that 7000 tonnes of organotin compounds have been used for non-pesticidal purposed in the year 2000, primarily as PVC stabilizers (Government of Canada, 2009). 
Because of the long persistence of TBT, effects of imposex (induced male sex organs on females) on molluscs in Canada had not been resolved by 1995, many years after regulation began (United Nations Environment Programme, 2006). By 2005, TBT was still considered a risk to freshwater organisms in Canada (Bartlett et al., 2005). The United Nations Environment Programme (2006) reports that TBT antifouling paints are permitted in Canada for vessels greater than $25 \mathrm{~m}$ in length, and vessels with aluminum hulls of any kind. As of March 2009, two tributyltin pesticide active ingredients were registered with Canada's PMRA and found in six end-use products (Government of Canada, 2009). Reductions in TBT concentrations have been found after regulations have been put in place, but not in locations still frequented by large vessels which continue to be applied legally with TBT-containing antifouling paints (Chau et al., 1997).

TBT is considered to be moderately to highly toxic to laboratory animals, and is able to cross the blood-brain barrier (United Nations Environment Programme, 2006). The freshwater environment is considered to be the most sensitive to TBT, because it has the most sensitive species and because it has a greater potential for accumulation due to lower rates of water exchange in lakes as compared to the open sea (United Nations Environment Programme, 2006). TBT was found to alter the activity of cytochrome P450 monooxygenases, which mediate testosterone metabolic processes (Oberdöster et al., 1998). Research on the negative environmental impacts of TBT is abundant. Recently concentrations as low as $1 \mathrm{ng} / \mathrm{L}$ have been found to induce imposex in female snails, and imposex, penis, and vas deferens in female gastropods, indicating it is a powerful endocrine disruptor. The mechanism of action is thought to be through disrupting endogenous P450 isozymes involved in steroid hormone hydroxylation (Oberdöster et al., 1998; Sekizawa et al., 2003). In crustaceans, TBT was found to be a teratogen and causes behavioural changes at $0.5 \mu \mathrm{g} / \mathrm{L}$, and at concentrations of $1.25 \mu \mathrm{g} / \mathrm{L}$ and above it affects reproduction and molting of D. magna (Oberdöster et al., 1998). At $2 \mathrm{ng} / \mathrm{L}$ it was found to cause increased shell calcification in the oyster Crassostrea gigas, and at $20 \mathrm{ng} / \mathrm{L}$ it was found to cause a decline in reproduction in other bivalve molluscs (Alzieu, 1998). At $6.6 \mu \mathrm{g} / \mathrm{L}$ it was found to alter the swimming behaviour of D. magna after 10 days (Schmidt et al., 2005), and $2.3 \mu \mathrm{g} / \mathrm{L}$ was the 1-week $\mathrm{LC}_{50}$ for $\mathrm{H}$. azteca (Borgmann et al., 1996). 
TBT will be used in tests with the automated image analysis systems as representative of the very persistent, bioaccumulating organometal class of contaminants.

\subsubsection{Copper}

Copper is a heavy metal that is an essential micronutrient for all living organisms, however at higher concentrations it is a known biocide, and especially an algicide (Hermens et al., 1984; Flemming et al., 1989; World Health Organization, 2004; Knauert \& Knauer, 2008; Ahmed \& Häder, 2010a). Copper sources in the environment include smelting, mining, industrial and agricultural waste, pesticides, wood preservatives, dyes, and many more (World health Organization, 2004; Ahmed \& Häder, 2010a), making this a ubiquitous compound. Many factors affect copper concentrations in water, including hardness and $\mathrm{pH}$. Under the Ontario Drinking Water Standards, the maximum concentration of copper is $1 \mathrm{mg} / \mathrm{L}$, and it is stated that this is an aesthetic objective only, to prevent an unpalatable taste in water (Ministry of Environment, 2001), although according to the WHO, copper was found at concentrations higher than the aesthetic objective in 53\% of homes in Nova Scotia (WHO, 2004), although generally concentrations are found under this limit.

Copper is ubiquitous in the environment and is found frequently in surface and ground waters (Health Canada, 2002). It is suggested that copper may act through the same mechanism as for dissolved oxygen in the water by affecting metabolic processes which regulate the internal oxygen concentration in exposed aquatic organisms (Untersteiner et al., 2003). Its toxicity to fish has been associated with disruption of sodium transport, and in D. magna it was found to impair feeding and inhibit growth and reproduction (Barata et al., 2006). The $48 \mathrm{~h} \mathrm{LC}_{50}$ for Daphnia spp. was found to be between 5 and $86 \mu \mathrm{g} / \mathrm{L}$ (Flickinger et al., 1982). Copper was also found to suppress cell growth, photosynthesis, chlorophyll synthesis and motility in algae through a variety of toxicity mechanisms like replacement of essential metals, inhibition of enzymes and oxidative damage (Knauert \& Knauer, 2008; Ahmed \& Häder, 2010a). It is especially important to assess copper toxicity using biomonitoring methods, as it has been shown to 
be dependent on a variety of water quality characteristics such as hardness, $\mathrm{pH}$, and organic matter content (Erickson et al., 1996; Welsch et al., 2000; de Schamphelaere \& Janssen, 2002; Ryan et al., 2009). Copper is also known to have synergistic effects on organisms, especially with other heavy metals such as zinc and cadmium (Hermens et al., 1984; Barata et al., 2006). This makes chemical analysis of copper concentrations effectively irrelevant to the environment and organism health, stressing the importance of biomonitoring systems to be sensitive to copper.

Copper will be used in tests with automated image analysis systems as representative of the water-soluble, heavy metal class of contaminants.

\subsubsection{DMSO}

Dimethyl Sulfoxide, or DMSO, will be used in experiments with atrazine and TBT as an organic carrier solvent. Organic solvents are considered by many to be necessary in aquatic ecotoxicology research to solubilise hydrophobic substances (LeBlanc \& Surprenant, 1983; Haap et al., 2007). In aquatic toxicity testing, standardized procedures recommend that solvents not be used in excess of $0.5 \%$ during acute toxicity tests and $0.1 \%$ during longer term toxicity tests (LeBlanc \& Surprenant, 1983). DMSO is commonly used in aquatic ecotoxicology to dissolve pesticides and herbicides due to these substances' low water solubilities (Stratton, 1985). It was found to be a suitable solvent in ecotoxicological studies using algae, fungi, and other bioassay organisms (Bowman et al., 1981; Haap et al., 2007). DMSO has been previously found to be the least toxic organic solvent to D. magna (Bowman et al., 1981; Stratton, 1985) and phytoplankton assemblages (Bérard, 1996) out of a variety of solvents such as methanol, ethanol, and acetone.

DMSO will be used at a concentration of $0.1 \%$ in tests using atrazine and TBT. Results of these tests will be compared to a control containing 0.1\% DMSO as well to ensure no toxic effects of the carrier. 


\subsection{Objectives}

The objectives of this study are: 1) to assess the suitability of the image analysis systems ECOTOX and DaphniaTox for implementation in a biological early warning system for drinking water in Ontario; 2) to investigate whether the organisms used are suitable bioassay organisms for these image analysis systems; and 3) to assess whether the sublethal behavioural parameters of these organisms as measured by the image analysis systems are suitable for detecting toxic stress caused by the contaminants and concentrations tested.

To assess the suitability of the image analysis systems, results of behavioural analysis using the systems were compared to visual observation of the behaviour of the organism in question. The sensitivities and repeatability of visual observation tests versus automated tests of E. gracilis were compared.

The following organisms were cultured in the lab: E. gracilis, D. magna, $H$. azteca, B. calyciflorus, and B. havanensis. Visual observation of E. gracilis behaviour was compared to the measured values produced by the automated system ECOTOX to determine whether the automated system is comparable and capable of detecting all behavioural changes. D. magna and $H$. azteca were used with the automated system DaphniaTox and the suitability of the system were determined based on results of tests with these organisms as well as ease of use and reliability of the system. The rotifers $B$. calyciflorus and B. havanensis have not been established as a standard bioassay organism

in Canada and as such their suitability for use in an early warning system for drinking water monitoring was assessed based on ease of culturing in the lab and applicability to use with the image analysis system ECOTOX. 


\subsection{MATERIALS AND METHODS}

This section describes how experiments were carried out. The culturing of organisms used in these experiments, E. gracilis, D. magna, H. azteca, B. calyciflorus and $B$. havanensis are described. Cleaning procedures, as well as hardware and software setup are also described. Experiments of visual observation and automated assessment of E. gracilis behavior after exposure to copper, atrazine, and TBT are detailed in this section.

\subsection{Culturing of Euglena gracilis}

Euglena gracilis was utilized in a variety of experiments using both observational methods with the aid of a microscope as well as the automated image analysis system ECOTOX, which measures a variety of behavioural parameters. Cultures were kept uniform for all experiments and cells were used in the medium. If experiments called for dilution of cell cultures due to a high density of cells, cultures were diluted with medium.

E. gracilis Z strain was cultured from an initial agar slant culture sample (Ward's Natural Science, 86W 2650) and grown in a liquid mineral medium developed by DonatPeter Häder (University of Erlangen-Nuremberg, Germany) and originally based on Checcucci's medium (Checcucci et al., 1976). The mineral medium (Appendix A) was prepared in $1 \mathrm{~L}$ batches where macronutrients and trace elements were combined in $1 \mathrm{~L}$ of Milli-Q ultrapure water and autoclaved at $121^{\circ} \mathrm{C}$ for 30 minutes. The $\mathrm{pH}$ was then adjusted to 6.8 as needed before the solution was autoclaved a second time for another 30 minutes at the same setting. The medium was rapidly cooled in a cold water bath to minimize precipitation, and once at room temperature, iron and vitamins were added aseptically to the mixture. The mineral medium was kept refrigerated for later use for up to 2 months in the lab. Before each use and before inoculation with new cells, the medium was left to adjust to room temperature. The hardness of the medium was calculated to be 94.4 ppm $\mathrm{CaCO}_{3}$ (Appendix B), which is important to note when considering results of copper toxicity experiments. 
Protocol for maintenance of cultures was based on procedures utilized for growth of Euglena gracilis at the Canadian Phycological Culture Centre (CPCC), formerly known as the University of Toronto Culture Collection of Algae and Cyanobacteria (UTCC) (J. Acreman, personal communication, August 2009). The medium was transferred to Erlenmeyer flasks and filled at $40 \%$ capacity, i.e. $100 \mathrm{~mL}$ of mineral medium in a $250 \mathrm{~mL}$ flask. Cells were transferred aseptically into the flasks, with a sterile inoculating loop if from the agar slant starter culture, or with disposable sterile pipettes if from pre-existing cultures at a ratio of 1:5 of algae culture and medium according to CPCC protocol. Flasks were covered with aluminum foil and placed on a continuous shaker at $100 \mathrm{rpm}$ at a room temperature of $24 \pm 2{ }^{\circ} \mathrm{C}$ under constant light using wide spectrum 40W "Plant \& Aquarium" Ecolux lamps with an intensity of 4000 lux (Environment Canada, 2007) at the surface of the flasks. Cultures between 7 and 10 days old were used for all experiments at a concentration of approximately $4.5-6.5 \times 10^{6}$ cells/mL, and cells were transferred into fresh medium between days 10 and 14 .

\subsection{Cleaning procedures}

All glassware was cleansed to remove traces of previously used contaminants prior to use. Aseptic techniques were used wherever appropriate, and laboratory working surfaces were kept clean with disinfecting solutions made and used according to Environment Canada's "General Procedures for Maintaining Sterile Conditions" (Environment Canada, 2007)

\subsubsection{Glassware}

All glassware, including Erlenmeyer flasks, beakers, vials, bottles and lids, was cleansed thoroughly to remove traces of previously used contaminants prior to use by rinsing in ethanol and dechlorinated municipal drinking water, and soaking for at least 15 minutes in Extran ${ }^{\circledR}$ detergent solution. Items were then scrubbed on all surfaces with a flask brush and rinsed in tap water until all traces of detergent were removed, and subsequently acid-washed in $10 \% \mathrm{v} / \mathrm{v}$ hydrochloric acid and rinsed three times with 
deionized water. All items were placed in an inverted position and left to air dry (Environment Canada, 2007). Glassware used for culturing E. gracilis, namely all Erlenmeyer flasks, bottles and lids, were sterilized prior to use by autoclaving at gravity cycle for a sterilization time of 15 minutes. Haemocytometers for the cell count experiment were washed with a strong jet of deionized water in between replicates and left to air dry. If dust was present after drying, the slides were cleaned off with lens paper.

\subsubsection{ECOTOX hardware}

The ECOTOX tubing and cuvette had to be periodically cleaned after periods of inactivity as well as after performing experiments with contaminants. Pure ethanol was pumped through the tubes and cuvette after finishing sets of experiments with the organic compounds TBT and atrazine, using the Rinsing setting on the ECOTOX software set to maximum speed (10) until $50 \mathrm{~mL}$ of ethanol flowed through. Following the ethanol, 100 $\mathrm{mL}$ of deionized water was pumped through the tubes and cuvette at maximum speed, with the additional "Rinsing" setting of "destroy bubbles" turned on to remove any bubbles which might impede in the visualization and mixing in the system. The same "Rinsing" setting at maximum speed was used in between replicates of experiments using $50 \mathrm{~mL} 0.1 \%$ DMSO solution. After days of inactivity, the tubes and cuvette were rinsed with deionized water using the "Rinsing" cycle at medium speed (5), for one minute, after which the speed was increased to maximum (10) with the "destroy bubbles" option checked, until $50 \mathrm{~mL}$ of water had been pumped through the tubes. Before each time the device was used, the tubes and cuvette were rinsed using the "Rinsing" cycle at maximum speed and "destroy bubbles" setting with $20 \mathrm{~mL}$ of deionized water. During the rinsing, tubes inside the ECOTOX hardware were checked to ensure that no leaks were present, and the software image on the computer screen was observed to ensure regular flow of fluid through the cuvette. 


\subsection{Contaminant dilutions}

Experiments were carried out by adding specific concentrations of contaminants to test organisms. The concentrations of contaminants used for the purpose of these experiments were very small, in the $\mu \mathrm{g} / \mathrm{L}$ range. For this reason, stock solutions were made for each contaminant, and dilutions from the stock were made for each concentration used in these experiments. These solutions were made to be $1000 \mathrm{x}$ concentrated for all contaminants.

Stock solutions were made of $500 \mathrm{mg} / \mathrm{L}$ atrazine and $100 \mathrm{mg} / \mathrm{L}$ tributyltin dissolved in dimethyl sulfoxide (DMSO) as a carrier solvent. A stock solution of $5 \mathrm{~g} / \mathrm{L}$ of copper sulphate was prepared in deionized water. Subsequent dilutions were made from these stock solutions to obtain $1000 \mathrm{x}$ concentrated solutions for each contaminant concentration used in experiments, again using DMSO for atrazine and TBT, and deionized water for copper, so that at each experiment the contaminant solution would be added at a 1:1000 v/v ratio to the E. gracilis-containing medium. For the contaminants being used with DMSO as a carrier, the final concentration of DMSO after this method would remain at $0.1 \%$, a concentration which has been found to be nontoxic (Bowman et al., 1981; LeBlanc \& Surprenant, 1983). Stock solutions of TBT were kept in a dark cupboard wrapped in aluminum foil to prevent photodegradation.

For experiments using copper as a contaminant, no DMSO was used; however, contaminant dilutions were still created using deionized water, to maintain a close similarity to the contaminant solution to growth medium ratio of 1:1000 used for experiments with atrazine and TBT. Copper was used as $\mathrm{CuSO}_{4} \cdot 5 \mathrm{H}_{2} \mathrm{O}$.

\subsection{Euglena gracilis cell motility experiments - visual observation}

The first behavioural parameter of E. gracilis examined by visual observation was cell motility, to be given as a percentage of motile cells. This category contains two discrete values, as cells are counted as either motile or nonmotile. 
Five $30 \mathrm{~mL}$ glass vials with lids were used per replicate of this experiment, which were filled with $20 \mathrm{~mL}$ of E. gracilis cell suspension in medium at a density of $4.5-6.5 \mathrm{x}$ $10^{6}$ cells $/ \mathrm{mL}$, and covered with loosely fitted lids and placed under a constant light source. Concentrations of contaminant added were based on previous findings by other studies (Marshall, 2009; Pearce, 2009).

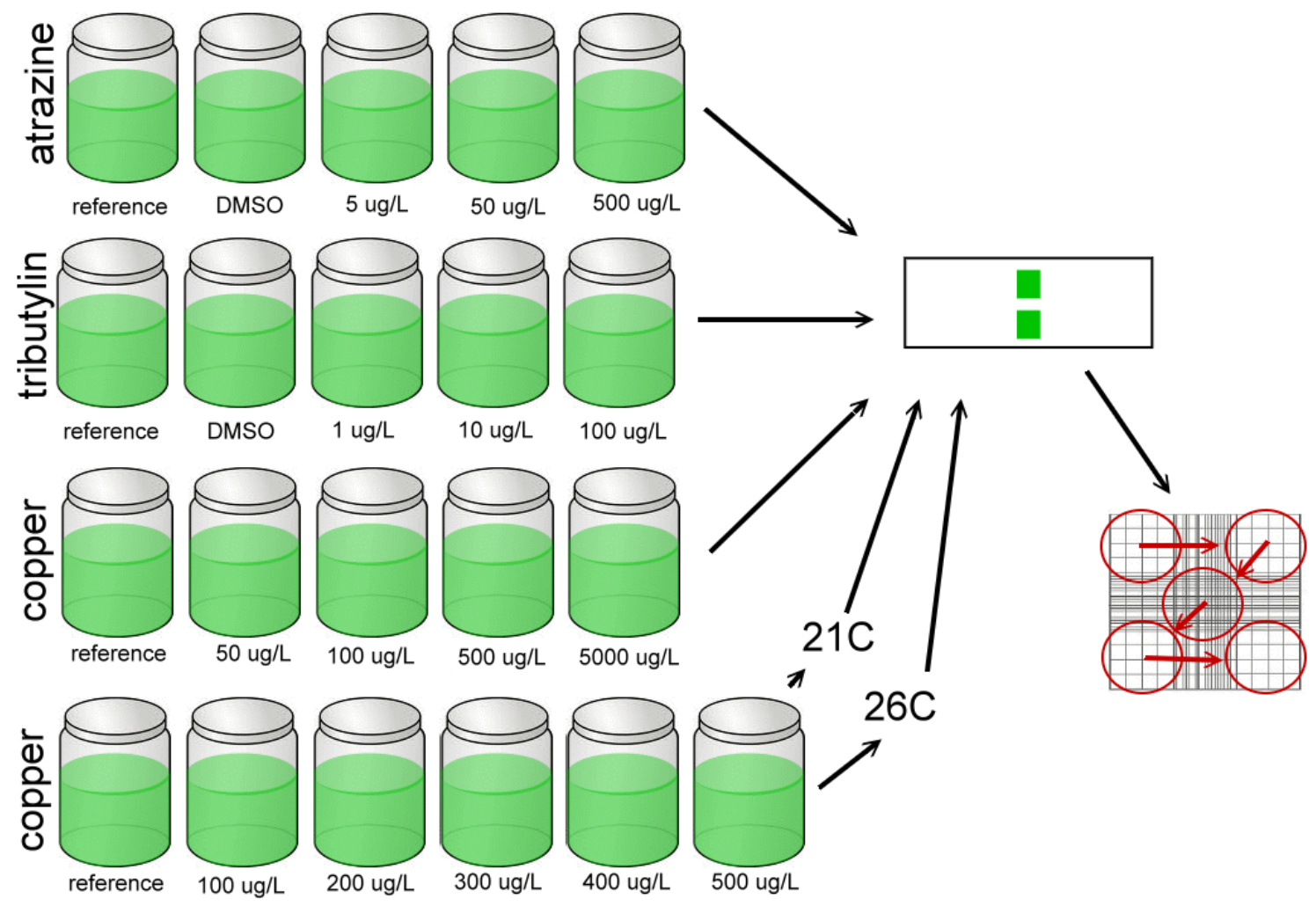

Figure 2.1. Experimental setup for cell motility experiments, where $20 \mathrm{~mL}$ of cell suspension in medium was added to vials with loosely fitted lids, and $20 \mu \mathrm{L}$ of $1000 x$ concentrated contaminant solution was added per vial. Treatments exposed to atrazine and TBT were in $0.1 \%$ DMSO carrier. Samples were taken and motility was measured after $1 \mathrm{~h}, 2 \mathrm{~h}$, and $24 \mathrm{~h}$ exposure to contaminant, except for the 100-500 $\mathrm{\mu g} / \mathrm{L}$ copper concentration range, where motility was measured after $1 \mathrm{~h}$ and $2 \mathrm{~h}$ incubation in copper.

Figure 2.1 shows a schematic breakdown of motility experiments. Five concentrations of contaminants, including reference and positive control, were tested as follows: contaminants were added from prepared stocks for each concentration. Atrazine and TBT were dissolved in DMSO in 1000x concentrated stocks, and from these $20 \mu \mathrm{L}$ were added to $20 \mathrm{~mL}$ of $\mathrm{E}$. gracilis cell culture placed in vials. The five different 
treatments for this experiment consisted of 23.2, 231.8, and $2318.2 \mathrm{nM}(5,50$, and 500 $\mu \mathrm{g} / \mathrm{L})$ for atrazine in $0.1 \%$ DMSO as a carrier; $1.7,16.8$, and $167.8 \mathrm{nM}(1,10$, and 100 $\mu \mathrm{g} / \mathrm{L}$ ) for tributyltin in $0.1 \%$ DMSO as a carrier; and in addition, one vial in reference conditions, and one vial in $0.1 \%$ DMSO carrier but no contaminant to ensure no toxic effects of this carrier. Stocks for copper were made in deionized water and concentrations were based on previous experiments looking at the effect of copper on Daphnia magna (A. Dort, personal communication, April, 2010). Stocks for copper were prepared from $\mathrm{CuSO}_{4} \cdot 5 \mathrm{H}_{2} \mathrm{O}$ and consisted of $0.2,0.4,2$ and $20 \mu \mathrm{M}$, corresponding to 12.7, 25.4, 127.2, and $1272.5 \mu \mathrm{g} / \mathrm{L}$ of $\mathrm{Cu}^{2+}$, the latter acting as a positive control. Sulfate $\left(\mathrm{SO}_{4}{ }^{2-}\right)$ has been previously found to have no toxic effects on the freshwater test species Daphnia magna and $\mathrm{CuSO}_{4}$ was therefore deemed an acceptable form of copper to be used (Erickson et al., 1996; de Schamphelaere \& Janssen, 2002).

During the experiment, cells were incubated in contaminant-containing solution for $1 \mathrm{~h}, 2 \mathrm{~h}$, and $24 \mathrm{~h}$. After each incubation period, vials were gently stirred to resuspend nonmotile cells immediately before a sample was taken from the vial using a micropipette, and placed on a haemocytometer with a slide cover for a cell count. Cells were counted immediately within 5 minutes of being placed on the haemocytometer to prevent migration of moving cells towards the edges of the well of the haemocytometer. All cell counts were rapid estimates, and slides were placed under the microscope at 400x magnification. The total number of cells was counted first with the aid of the haemocytomter grid and by dividing the area into quadrants, where cells were counted in groups of 10. Following this, the number of nonmotile cells was counted. Since nonmotile cells had a tendency to sink towards the bottom of the slide due to gravity, the focus of the microscope lens could be slightly changed with the fine adjustment so that nonmotile cells were in focus and motile cells were not. This allowed for a more reliable and faster cell count. Cells were again counted in groups of 10 and the total number of nonmotile cells was noted. Five observations and total and nonmotile cell counts were noted for each sample taken out of vials, from the four outer corners of the haemocytometer grid as well as the centre. Observations were made in a " $Z$ " sequence so as to minimize disturbance to the cells due to re-exposure to light from the microscope, as they are photosensitive (Figure 2.1). All experiments were replicated at least 5 times. 
Motility for each replicate was recorded by dividing the number of nonmotile cells by the total number of cells, determining the percentage of nonmotile cells, which was then converted to a percentage of motile cells by subtracting from 100 .

Following results from the copper experiment, the procedure was repeated at an expanded concentration range of copper between 100 and $500 \mu \mathrm{g} / \mathrm{L}$, to include 100, 200, 300,400 , and $500 \mu \mathrm{g} / \mathrm{L}$. This concentration range was chosen due to the lack of visible effects of motility at $100 \mu \mathrm{g} / \mathrm{L}$ and the strong, significant effects at $500 \mu \mathrm{g} / \mathrm{L}$. This experiment was performed to obtain a dose-response relationship between copper concentration and E. gracilis cell motility. The same procedure was repeated from previous motility experiments where vials with loosely fitted lids were filled with $20 \mathrm{~mL}$ of E. gracilis-containing medium and copper solution was added. Measurements were taken after $1 \mathrm{~h}$ and $2 \mathrm{~h}$ incubation in copper. Experiments were replicated 5 times at an ambient temperature of $20-21^{\circ} \mathrm{C}$ and 5 times at ambient temperature of $25-26^{\circ} \mathrm{C}$. The same procedure for counting total number of cells, nonmotile cells, and percent motility was used.

\subsection{Euglena gracilis cell shape experiments - visual observation}

The second behavioural parameter of E. gracilis to be investigated was cell shape, i.e. whether round or elongated. Changes in this behaviour can be seen instantaneously under a microscope.

E. gracilis cell shape was investigated under copper stress, as previous research at Ryerson University has already examined changes in cell shape after exposure to atrazine and TBT (Pearce, 2009). Procedures for this experiment have been based on experiments previously carried out and modified to reduce error. The concentration range was based on the previous motility experiment and matched with the automated analysis experiment for the purpose of comparability. 
As previously, vials with loosely fitted lids were filled with $20 \mathrm{~mL}$ of cell suspension in medium and $20 \mu \mathrm{L}$ of copper solution. The concentrations of copper exposed were $100,300,500$, and $5000 \mu \mathrm{g} / \mathrm{L}$, as well as one vial in reference conditions. Cells were exposed to copper solution for $1 \mathrm{~h}$ and $2 \mathrm{~h}$, after which a sample was taken from the vials and placed on a haemocytometer with a slide cover. Observations were taken within 60 seconds of placing sample on slide, and cells were observed at 4000x magnification. A Canon PowerShot A710IS camera was placed on the eyepiece of the microscope and picture stills were taken randomly at different areas of the slide within the counter grid until the first 12 cells were counted. Each cell was assigned a number value for shape category. These values were assigned as increasing with elongation so as to be comparable to the values for cell compactness given by the automated system, which is a form factor value based on circumference to area ratio. The values assigned to cell shapes in this observational experiment were as follows: 0 , for round cells; 1 , for oval cells; and 2, for elongated cells. This experiment was replicated three times.

\subsection{ECOTOX automated system experiments}

The automated image analysis system ECOTOX was used to detect behavioural changes in the test species E. gracilis, and the system's ability to detect behavioural changes in comparison to visual observation methods was assessed. ECOTOX was designed by Donat-Peter Häder (University of Erlangen-Nuremberg, Germany) specifically to be used with E. gracilis, although use with other species of flagellates is also possible (de Kuhn et al., 2006).

\subsubsection{Hardware setup}

The ECOTOX image analysis system consists of a hardware and a software component. Before each experiment, the system must be set up and prepared for use. A schematic diagram of the ECOTOX hardware system can be seen in Figure 2.2. 
a)

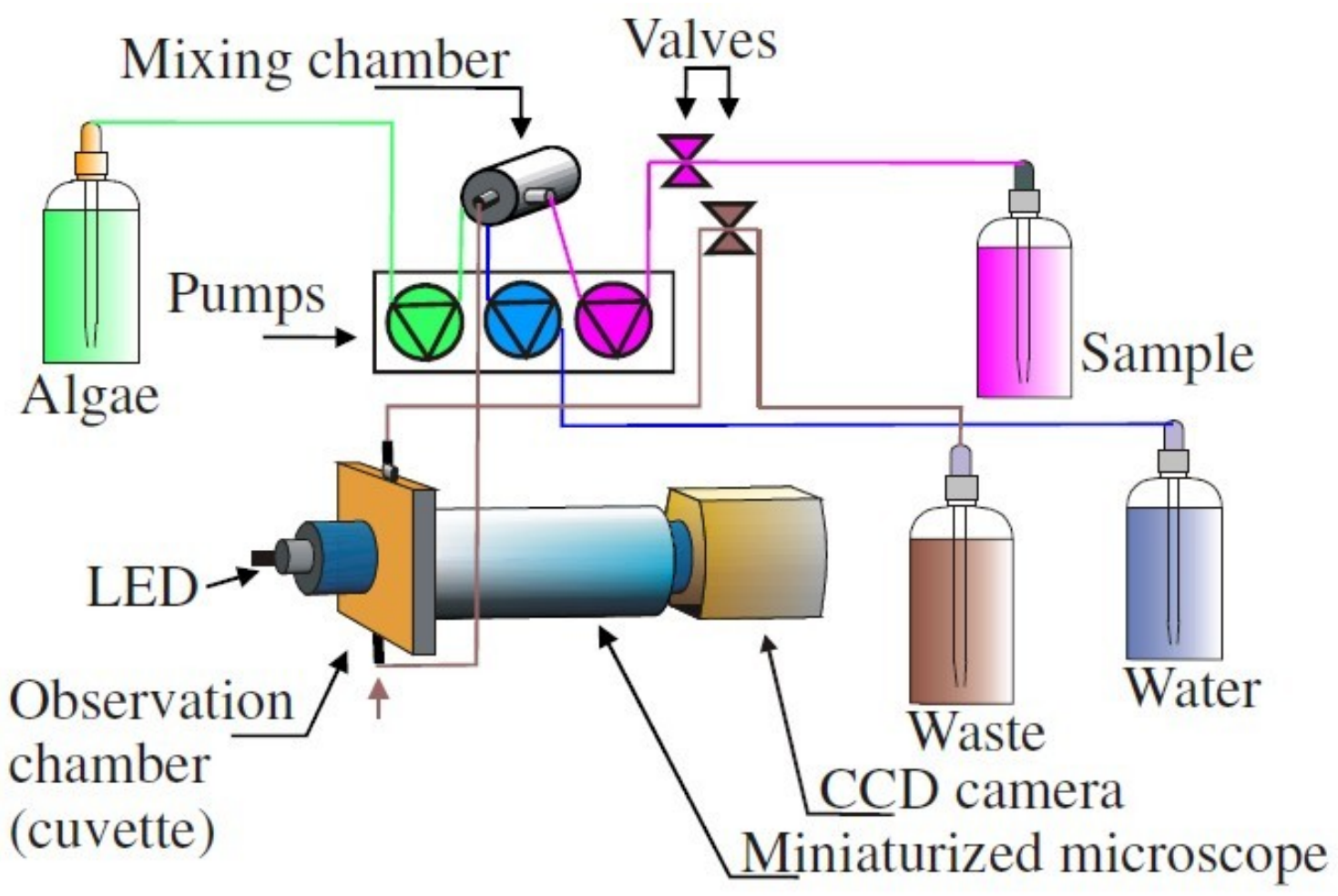

b)

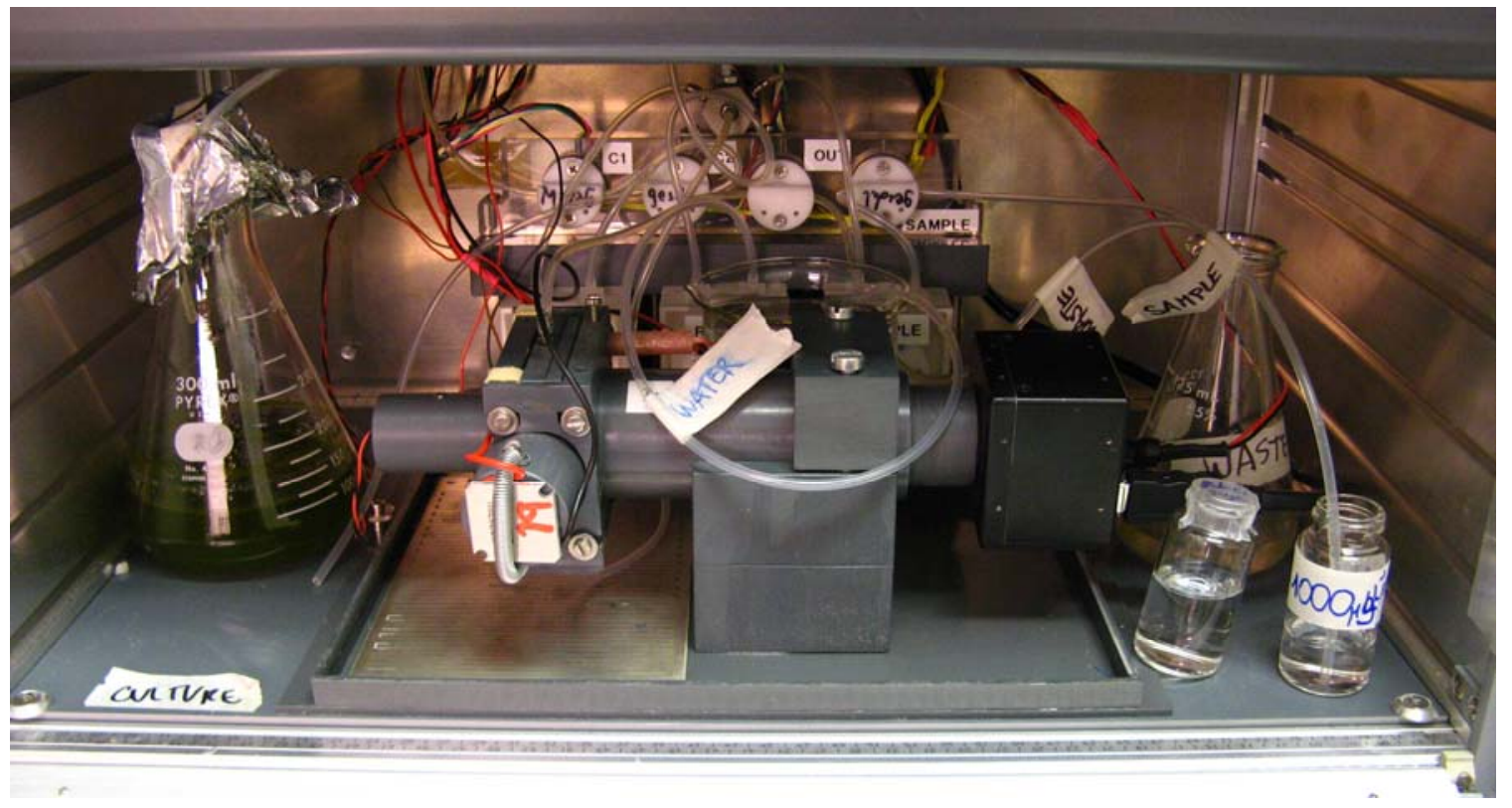

Figure 2.2. The ECOTOX hardware system, shown in a schematic diagram in a) where connections are seen between tubes, pumps, mixing chamber and cuvette, and in a photograph in b) where the equipment is set up and ready for use. 
The tubes containing E. gracilis culture, contaminant sample, and dilution water all connect to individual peristaltic pumps which are controlled by the software. The pumps lead the contents of the culture, water, and contaminant sample tubes to a mixing chamber, where all liquids are mixed and pumped as a single mixture towards the observation chamber (cuvette). The cuvette is in the vertical position and the solution mixture is pumped upwards from the bottom, therefore the software will produce images of an upwards flow of cells during pump rotation.

The ECOTOX hardware box must be plugged in and the power switch must be turned on. A Firewire cable and a VGA cable must both be connected to a computer. An empty $250 \mathrm{~mL}$ Erlenmeyer flask was then placed inside the system where the tube labelled "waste" was inserted. A $200 \mathrm{~mL}$ beaker filled with dilution water was then placed in the system, containing either E. gracilis mineral medium for copper experiments or $0.2 \%$ DMSO for atrazine and TBT experiments, and the tube labelled "water" was inserted into this beaker, ensuring that the tube touched the bottom of the beaker. This water is used for diluting the cell culture and contaminant solution in all experiments, as well as rinsing the system (tubing and observation cuvette) in between readings. A $50 \mathrm{~mL}$ beaker was placed in the system next, containing contaminant solution at $2 \mathrm{x}$ the maximum concentration of contaminant necessary for the experiment, and $0.2 \%$ DMSO for experiments with atrazine and TBT. The tube labeled "sample" was inserted into this beaker, ensuring that the end of the tube reached the bottom of the beaker. Finally, a graduated cylinder containing E. gracilis in mineral medium at the appropriate density was placed on the left-centre inside the system, where the tube labeled "culture" was inserted two-thirds of the way down to the bottom. The cell culture tube was not pushed towards the bottom of the cylinder to prevent an inadequately high cell density inside the ECOTOX tubes, as cells settle down to the bottom and creates a density gradient. For this reason the cylinder was also gently stirred in between experiments to resuspend cells that had settled to the bottom. All the tubing inside the system was then checked for air bubbles and to ensure stable connections. 


\subsubsection{Software setup}

Once plugged in, set up and connected, the ECOTOX software can be run from the computer. If the software is opened when the system is turned off or disconnected, an error message will appear. During experiments all tubes were checked for possible leaks, and the flask with waste material was checked to prevent overflowing and emptied periodically. The software image displayed on the computer screen was observed throughout all experiments to ensure that the flow was regular, that there was no contamination of the sample, and that the cell movement was being tracked appropriately. Cells being tracked are highlighted in green, and movement which detected but does not fit cell size specifications is highlighted in red.

Specific software settings for all tests were: minimum swimming speed $10 \mu \mathrm{m} / \mathrm{s}$; 60 seconds rinsing time of cuvette, 30 seconds filling time for control and sample, 60 seconds incubation time in the dark.

\subsubsection{Controlled trials with ECOTOX}

The ECOTOX system is capable of three recording modes. The "Control" mode simply performs a one-time reading of cells diluted in a 1:1 ratio with sample solution (containing either reference water or contaminant sample). The "Single-Toxin" mode performs serial dilutions by mixing dilution water and sample solution, and is described in more detail in section 2.6.4. Finally, the "On-line" mode takes measurements at set intervals and was designed to be used with a continual readings test such as would be used with a flow-through system in a water quality testing setting.

To test for the appropriate functioning of the ECOTOX equipment, cell readings were taken using the "Control" mode, which dilutes cell cultures in a 1:1 ratio with sample water. Several control solutions were used: E. gracilis growth medium, distilled water with $0.2 \% \mathrm{DMSO}, 50 \mathrm{~g} / \mathrm{L} \mathrm{NaCl}$, and $100 \%$ ethanol. Due to the dilution ratio, final exposures of E. gracilis were: 100\% growth medium, 0.1\% DMSO, $25 \mathrm{~g} / \mathrm{L} \mathrm{NaCl}$, and $50 \%$ Ethanol. The $\mathrm{NaCl}$ and ethanol treatments were included as positive controls. The 
DMSO treatment was included to ensure no effects on the organism from the organic carrier being used in experiments with atrazine and TBT. All experiments were repeated three times.

\subsubsection{Serial dilutions setup}

Under the Single Toxin recording mode in ECOTOX, automatic serial dilutions are performed where software-controlled pumps bring together the cell-containing medium, the contaminant solution, and deionizer water used for dilution. The values for the serial dilutions were acquired from Michael Lebert and Donat-Peter Häder from the University of Erlangen-Nuremberg, Germany (M. Lebert, personal communication, November 2009). The value of contaminant solution to total volume inside the ECOTOX cuvette was determined to be $6 \%, 12 \%, 21 \%, 34 \%$, and $50 \%$ (Figure 2.3 )

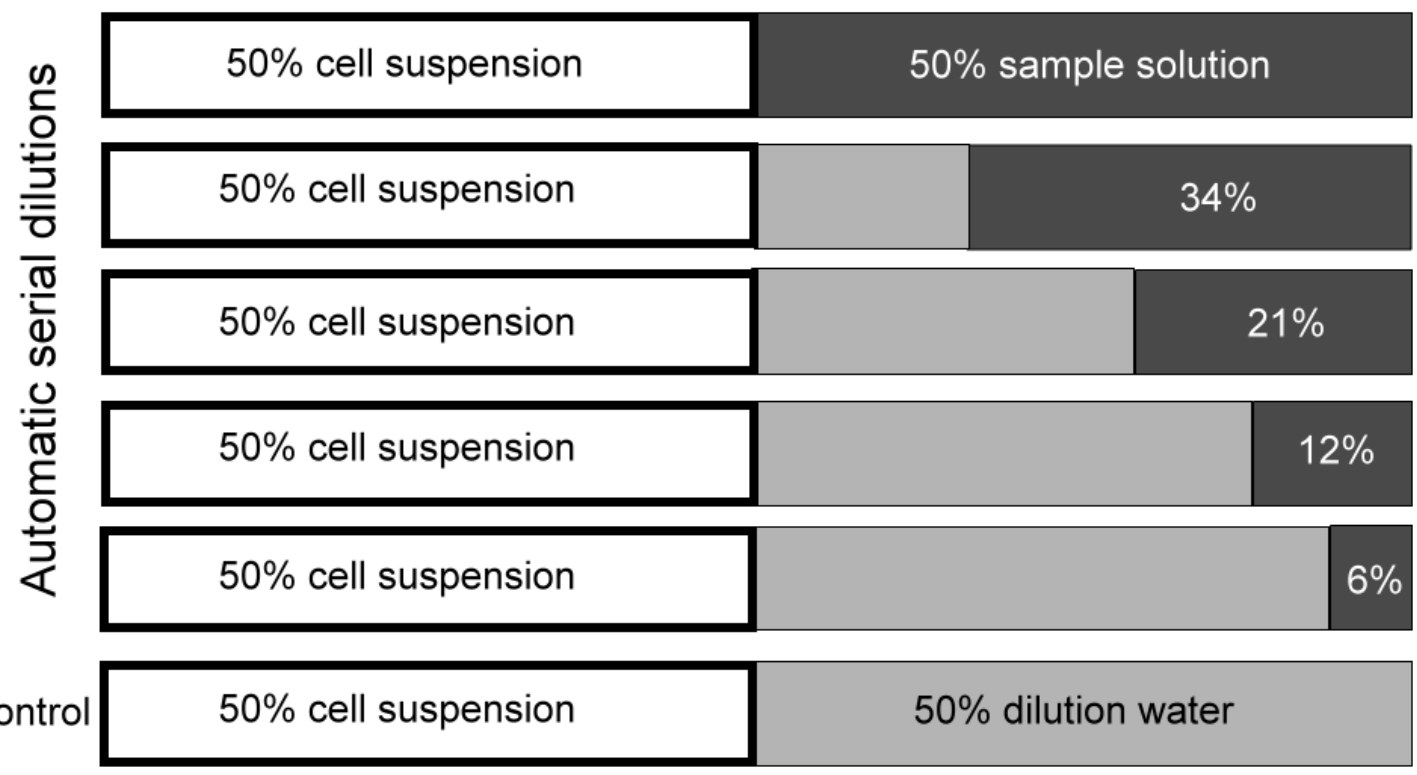

Figure 2.3. Proportional diagram of volumes of cell suspension in medium, water, and contaminant solution in the cuvette after performing a serial dilution experiment in Single Toxin mode. 
The experiments with atrazine and TBT were designed to be used with an amount of $0.1 \%$ DMSO in total volume throughout all replicates. To achieve this and maintain levels of DMSO constant between the automatic dilutions and control when in use, both the sample solution and the dilution water contained 0.2\% DMSO. When these were diluted with the constant $50 \%$ proportion of cell suspension, the total proportion of DMSO per total volume is $0.1 \%$ as intended inside the cuvette. An additional reading of cells under Control setting, which measured a standard 1:1 cell suspension and dilution water was performed without DMSO to ensure no toxic effects of this carrier.

The settings of the software were set at maximum incubation time in the dark, i.e. 5 minutes, which is all the time the cells were exposed to the contaminant before the system began recording behavioural responses. The tracking time was set to 60 seconds after incubation in the dark. Further timed settings included the filling time of the cuvette which was set to 30 seconds, and the filling time for the control sample and rinsing, which was set at 40 seconds.

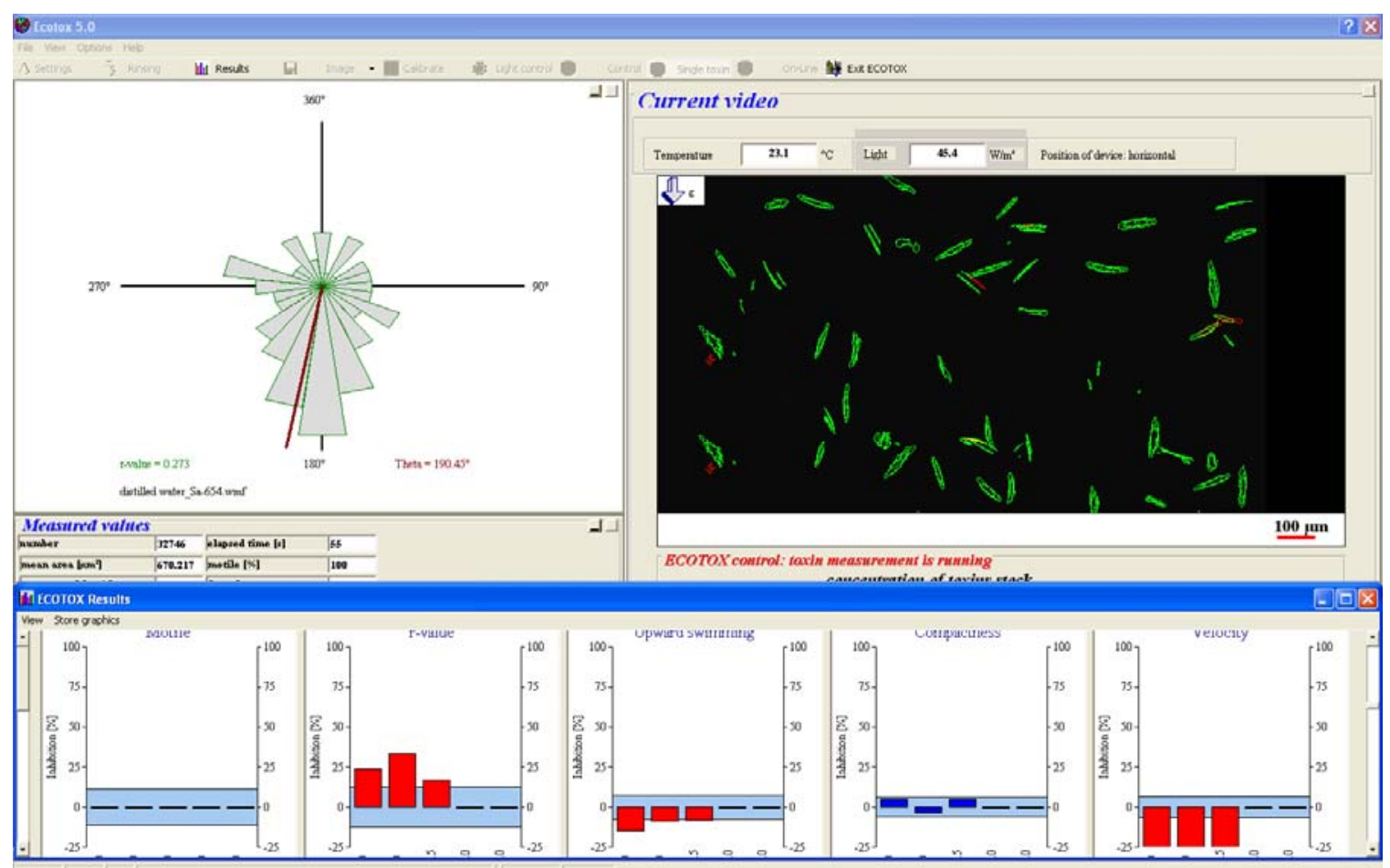

Figure 2.4 ECOTOX software screenshot showing circular histogram (top left), observation chamber with cells being tracked (green) and objects not being tracked (red), and bar charts with percent inhibition of parameters (bottom). 
Results were given as percent inhibition compared to control, and the software generated bar graphs on screen in real-time such as shown in Figure 2.4. Inhibition values given were not used for the purpose of this research. Raw data for these experiments is also available in the software-generated ASCII files with an ".ect" file extension which can be opened with MS Excel using comma delineated values. The parameters are shown in columns and the various measurements are recorded in the rows, with an inhibition value compared to control is shown on the bottom of each dilution series. The averages and standard deviations for each parameter at each concentration were recorded.
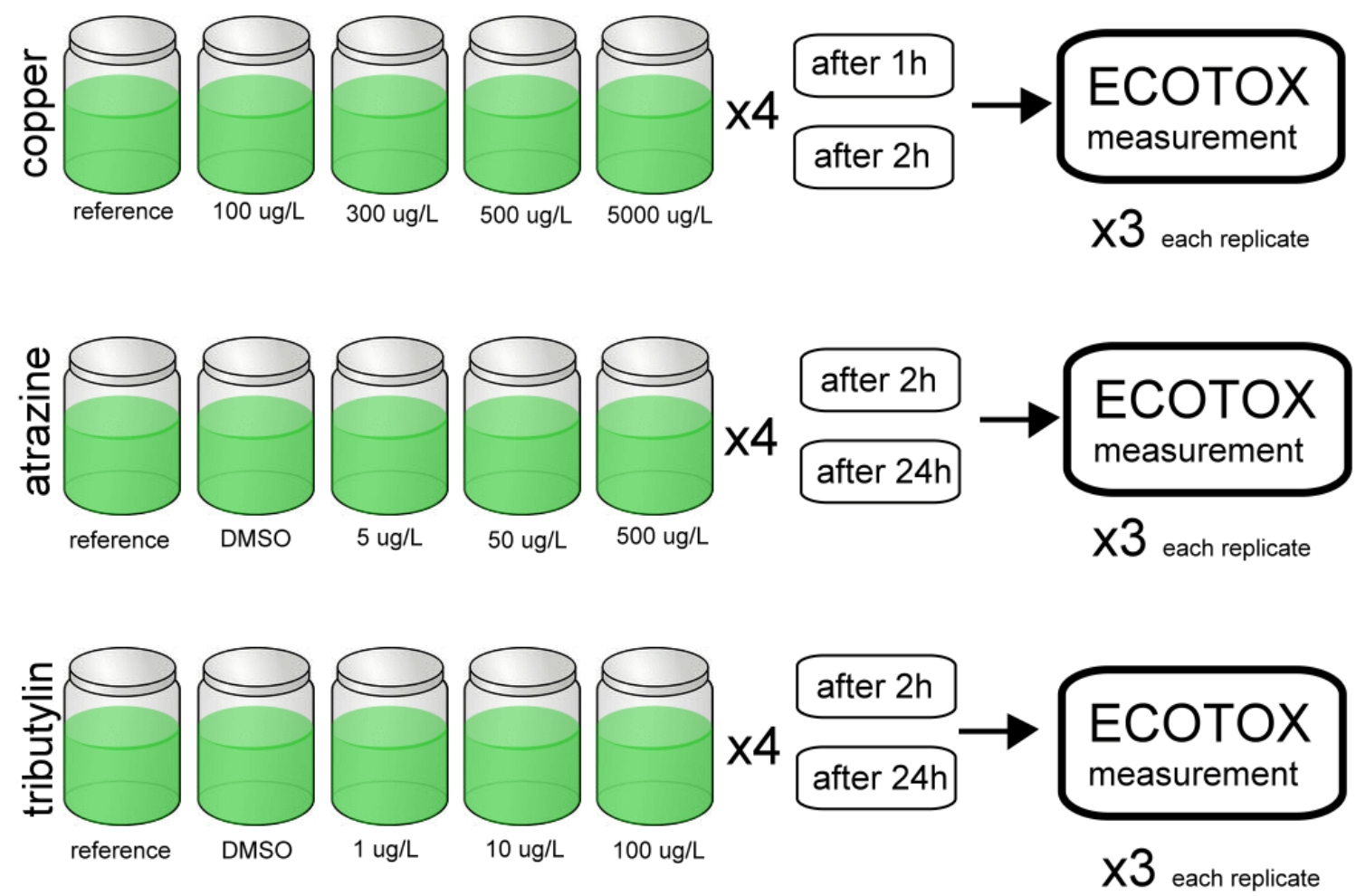

Figure 2.5. Experimental setup for the ECOTOX measurement experiments, where $20 \mathrm{~mL}$ of cell suspension in medium was added to vials with loosely fitted lids, and contaminant was added at a ratio of 1:1000 $(20 \mu \mathrm{g} / \mathrm{L})$ from individually prepared stocks for each concentration. Stocks for copper sulphate were prepared in deionized water, whereas stocks for atrazine and tributyltin were prepared in DMSO, giving a final concentration of $0.1 \%$ DMSO throughout treatments. 


\subsubsection{Standard dilutions and incubation setup}

Like the cell motility experiments, for this experiment of automated behaviour readings with ECOTOX, five $30 \mathrm{~mL}$ glass vials with lids were used per replicate, which were filled with $20 \mathrm{~mL}$ of E. gracilis cell suspension in medium at a density of $4.5-6.5 \mathrm{x}$ $10^{6}$ cells $/ \mathrm{mL}$. Concentrations of contaminant were chosen as to enable comparison to the motility experiments. Figure 2.5 shows a breakdown of treatment for each contaminant, where in the copper experiments cells were exposed to $100,300,500$ and $5000 \mu \mathrm{g} / \mathrm{L}$, as well as reference conditions with no contaminant; in the atrazine experiment cells were exposed to 5,50 , and $500 \mu \mathrm{g} / \mathrm{L}$ in $0.1 \%$ DMSO as well as treatment in $0.1 \%$ DMSO and reference conditions with no contaminant; in the Tributyltin experiment cells were exposed to 100,10 , and $1 \mu \mathrm{g} / \mathrm{L}$ in $0.1 \%$ DMSO as well as treatment in $0.1 \%$ DMSO alone and reference conditions with no contaminant.

Cells were then placed under a constant light bank with the lids loosely fitted for an incubation period of $1 \mathrm{~h}$ and $2 \mathrm{~h}$ for copper, and $2 \mathrm{~h}$ and $24 \mathrm{~h}$ for atrazine and TBT based on results from motility experiments, which are described in section 2.4 and discussed in section 3.1. Once incubated, the vials were placed into the ECOTOX hardware box, and the tube connected to the "algae suspension" pump was inserted into the vial. The "Rinsing" cycle was turned on briefly through the software at maximum speed for the "algae" pump only because of the extensive length of the culture tube and to ensure that once the recordings were turned on the cell suspension would enter the mixing chamber. To begin measurements, the "Control" recording setting was used, which mixes cell suspension and dilution water at a 1:1 ratio. This does not affect the readings as the cells have separately incubated in the appropriate amount of contaminant and carrier if applicable. The "dilution water" used for all experiments was itself the mineral growth medium for E. gracilis, which was necessary to maintain consistency with parallel experiments. Software specifications for the experiments were set to 60 second incubation in the dark in the cuvette, a 60 second tracking period, 40 second rinsing time of the cuvette, and 30 second filling time for measurements. Raw data for these experiments were generated as ASCII files with an ".ect" file extension which were be opened with MS Excel using comma delineated values. The parameters measured by the 
ECOTOX system investigated in this study were: i) cell swimming velocity, ii) r-value or randomness of swimming, iii) cell compactness or shape, and iv) percent number of cells swimming upwards. Other parameters produced by the automated system were not studied.

To compare visual observations with parameters measured by the automated system, assumptions were made that the observational parameters were comparable to the automated parameters. Two of the parameters compared were visually observed motility and the automated velocity measurement. It was assumed that motility and velocity would be equivalent, as the automated velocity value is given as an average of many cells over a 60 second period, and the lower the motility, the lower the overall velocity of cells would be, as well as the higher the motility, the closer the velocity would be to the average velocity at reference conditions. Despite the ECOTOX software producing a value for cell motility as well as cell velocity, this value did not correspond to observations on the screen of the software showing cell movement, as this value was always given as $100 \%$. Therefore, this value was not considered when comparing automated methods with visual observations. It was also assumed that the visual observations of cell shape, measured with an assigned value for each cell shape, would be comparable and equivalent to the automated measurement of cell compactness, given as cell form factor, a ratio of circumference to area, which was also lower for rounder cells and higher for more elongated cells.

\subsection{Culturing of Daphnia magna}

Daphnia magna was cultured to be used for experiments using the DaphniaTox image analysis system, developed by Donat-Peter Häder (University of ErlangenNuremberg, Germany), which looks at various behavioural parameters using the same principles as the ECOTOX image analysis system. 
Cultures of Daphnia magna were maintained based on Environment Canada guidelines (1996). A 9-litre aquarium tank was filled with $7 \mathrm{~L}$ of natural spring water (Selection brand, Metro Inc.) at 395 ppm $\mathrm{CaCO}_{3}$ hardness (Appendix B). The depth of the water was maintained at approximately $15 \mathrm{~cm}$ after evaporation with the same natural spring water used to start the tank. The water was aerated with a bubbler for $48 \mathrm{~h}$ to ensure oxygen saturation prior to adding D. magna to the tank. The tank was maintained at laboratory room temperature, which ranged at $22 \pm 2^{\circ} \mathrm{C}$ throughout the duration of experiments. The tank was kept aerated with the aid of an air bubbler kept near the surface of the water to prevent physical stress form vigorous bubbling, and was placed under cool white light at 500 lux at a 16:8 hours light to dark cycle. An initial number of 20 D. magna were placed in the tank. Feedings were carried out three times a week where 6-8 drops of Roti-Rich Invertebrate Food (Ward's Natural Science) and 10-15 mL of Pseudokirchneriella subcapitata culture $\left(3 \times 10^{6}\right.$ cells $\left./ \mathrm{mL}\right)$ were added. The tank was observed for water clarity biweekly, and if necessary the water was partially removed and replaced with fresh aerated natural spring water.

\subsection{Culturing of Hyalella azteca}

Hyalella azteca was cultured to be used for experiments using the DaphniaTox image analysis system on experiments equivalent to the D. magna experiments using DaphniaTox.

Cultures of Hyalella azteca were maintained based on Environment Canada guidelines (1997). A 9-litre aquarium tank was filled with $7 \mathrm{~L}$ of dechlorinated Toronto municipal tap water, with a hardness of between 106 and $127 \mathrm{ppm} \mathrm{CaCO}_{3}$ according to the City of Toronto (2010). The depth of the water was maintained at approximately 15 $\mathrm{cm}$ after evaporation with the same dechlorinated municipal tap water used to start the tank. The water was aerated with a bubbler for $48 \mathrm{~h}$ to ensure oxygen saturation prior to adding $H$. azteca to the tank. The bottom of the tank was lined with sterile gauze, which was replaced every 2 months. The tank was maintained at laboratory room temperature, 
which ranged at $22 \pm 2^{\circ} \mathrm{C}$ throughout the duration of experiments. The tank was kept aerated with the aid of an air bubbler kept near the surface of the water to prevent physical stress form vigorous bubbling, and was placed under cool white light at 500 lux at a 16:8 hours light to dark cycle. An initial number of $20 \mathrm{H}$. azteca were placed in the tank. Feedings were carried out three times a week where 5-6 flakes of TetraMin ${ }^{\circledR}$ Flakes fish food and 10-15 mL of Pseudokirchneriella subcapitata culture $\left(3 \times 10^{6}\right.$ cells $\left./ \mathrm{mL}\right)$ were added. The tank was observed for water clarity biweekly, and if necessary the water was partially removed and replaced with fresh aerated and dechlorinated municipal tap water.

\subsection{Culturing of Pseudokirchneriella subcapitata for invertebrate feeding}

Cultures of Pseudokirchneriella subcapitata, previously known as Selenastrum capricornutum, were maintained for the purpose of feeding and maintaining D. magna and H. azteca cultures.

P. subcapitata was cultured according to Environment Canada guidelines (1997). Five stock solutions were made (Appendix C), where $500 \mathrm{~mL}$ of Millipore water was added to the nutrient compounds at the amount specified. Solutions were then filtersterilized at a vacuum through a $0.4 \mu \mathrm{m}$ membrane. Solutions were non-autoclaved according to Environment Canada recommendations for optimal growth. To make the growth medium, $1 \mathrm{~mL}$ of each stock solution was added in sequence to $999 \mathrm{~mL}$ of Millipore water and mixed in a volumetric flask (1:1000 dilution of stock). Contents were then distributed in $250 \mathrm{~mL}$ Erlenmeyer flasks filled at a volume-to-flask ratio of $20 \%$ (i.e. $50 \mathrm{~mL}$ in a $250 \mathrm{~mL}$ flask) to avoid carbon monoxide accumulation. An initial batch of algae was grown from a Selenastrum capricornutum algae slant culture (Ward's Natural science, 86V 0620) using an inoculating loop, and subsequent cultures were inoculated from existing liquid cultures at a 1:50 ratio, i.e. $1 \mathrm{~mL}$ of algae culture for $50 \mathrm{~mL}$ of growth medium. 


\subsection{DaphniaTox automated system experiments}

The automated image analysis system DaphniaTox was used to detect behavioural changes in the test species D. magna and H. azteca, and the system's ability to detect behavioural changes was assessed. DaphniaTox was designed by Donat-Peter Häder (University of Erlangen-Nuremberg, Germany) specifically to be used with D. magna, although it can also be adapted for use with other macroinvertebrates.

\subsubsection{Hardware and software setup}

The DaphniaTox image analysis system consists of a hardware and a software (Figure 2.6) component. Before each experiment, the system must be set up and prepared for use.

The DaphniaTox hardware box must be plugged in, and once it is plugged in it is automatically on, as there is no power switch. The Firewire cable and the VGA cable must be connected to the computer. Once the hardware is plugged in and connected, the software can be run from the computer. Two $250 \mathrm{~mL}$ polystyrene canted neck tissue culture flasks containing organisms in the appropriate treatment were then placed on the platform flask holder in front of the camera pushed toward the LED light source. The flasks must be placed so that they can be both equally seen on the software display on the computer screen. From the software, the screen image from the camera was adjusted using the red LED light adjustments, brightness and contrast adjustments until only the organisms were clearly marked and tracked, avoiding glare and dust particles. A panel covering the red LED lights inside the hardware box can also be adjusted to avoid reflective glare on the screen. From the software, the number of flasks in use must be selected under "Cuvette Usage". For the purpose of these experiments, two flasks, or cuvettes, were always used. Once the two flask usage option was determined, the Area of Interest, or AOI, must be selected. This option allows the user to select on the screen the area of the image which will be analyzed for movement. A maximum area of the flasks which was visible on the screen was selected. Once set up, experiments could begin. 


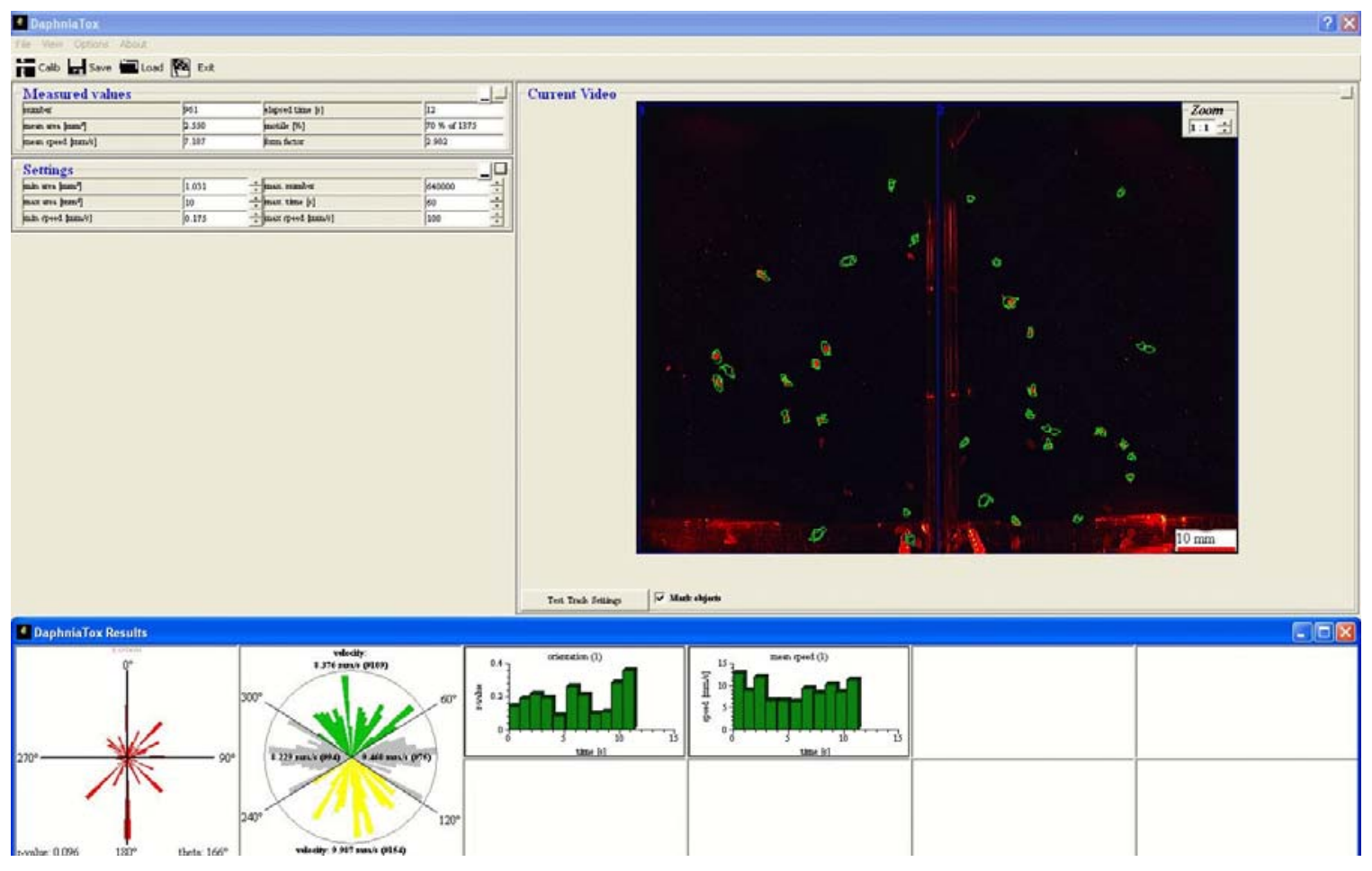

Figure 2.6 DaphniaTox software screenshot showing observation chamber with 2 flasks separated by a blue line and in each $D$. magna being tracked (green), circular histogram of movement (bottom left), and bar charts with per-minute average values of swimming velocity (bottom right).

\subsubsection{Daphnia magna experiments}

The behaviour of Daphnia magna under atrazine stress was examined using the DaphniaTox system. A $250 \mathrm{~mL}$ polystyrene canted neck tissue culture flask was filled with natural spring water (Selection brand, Metro Inc.) and 15 indivduals between 7-10 days old were inserted in the flasks using a transfer pipette with the narrow end cut off. Neonates were not used for these experiments because they are too small to be detectable by the image analysis system. Individuals were left to adapt inside the flask for 30 minutes before addition of contaminant. Seven different treatments were used: Reference (natural spring water only), 0.1\% DMSO, and 5, 25, 50, 250, and $500 \mu \mathrm{g} / \mathrm{L}$ of atrazine in $0.1 \%$ DMSO as a carrier solvent. Contaminants were added from previously made solutions $1000 x$ concentrated in DMSO at a volume of $250 \mu \mathrm{L}$. Two separate flasks with 
two treatment conditions were prepared and placed inside the DaphniaTox hardware for a further 30 minutes for red LED light adaptation and incubation in contaminant. After this adaptation period, the measurements were then started using the software, where tracking time was set to 60 seconds every 10 minutes.

\subsubsection{Hyalella azteca experiments}

The behaviour of Hyalella azteca was examined using the DaphniaTox system. A $250 \mathrm{~mL}$ polystyrene canted neck tissue culture flask was filled with dechlorinated municipal tap water and 15 indivduals between 2 and 3 weeks old, or 3 to $5 \mathrm{~mm}$ in length, were inserted in the flasks using a transfer pipette with the narrow end cut off. Environment Canada (1997) recommends using individuals 7-10 days old for acute survival and growth toxicity tests. However these were not used for the DaphniaTox experiments because they are too small to be detectable by the image analysis system. Individuals were left to adapt inside the flask for 30 minutes before addition of contaminant, and no substrate was used in the flasks. Seven different treatments were used: Reference (natural spring water only), 0.1\% DMSO, and 5, 25, 50, 250, and 500 $\mu \mathrm{g} / \mathrm{L}$ of atrazine in $0.1 \% \mathrm{DMSO}$ as a carrier solvent. Contaminants were added from previously made solutions $1000 x$ concentrated n DMSO at a volume of $250 \mu \mathrm{L}$. Two separate flasks with two treatment conditions were prepared and placed inside the DaphniaTox hardware for a further 30 minutes for adaptation in the ambient red LED light inside the system (which does not produce a phototactic response in D. magna) and incubation in contaminant. After this adaptation period, the measurements were then started using the software, where tracking time was set to 60 seconds every 10 minutes.

\subsection{Culturing of Brachionus spp.}

Brachionus spp. rotifers were cultured in the lab to investigate their possible use as bioassay organisms as well as possible use with the ECOTOX image analysis system. 
Cultures of Brachionus calyciflorus and Brachionus havanensis were grown indvdually in mixed cultures with Chlorella vulgaris. Samples were obtained from Alison Derry (McGill University, Motreal). Brachionus havanensis cultures were originally sourced from S.S.S. Sarma (University of Mexico), and Brachionus calyciflorus cultures were originally sourced from Gregor Fussman (Department of Biology, McGill University, Motreal). Cultures were grown in a modified COMBO medium (Kilham et al., 1998) in $50 \mathrm{~mL}$ beakers under constant light partially covered with parafilm (Appendix D). Cultures were transferred into new medium every 3 weeks at a 1:1 ratio.

COMBO medium was made from ten stock solutions, consisting of seven major macronutrient stocks ( $100 \mathrm{~mL}$ was made of each), one algae trace elements stock (500 $\mathrm{mL})$, one animal trace elements stock $(500 \mathrm{~mL})$, and one vitamins stock $(100 \mathrm{~mL})$. Stocks were kept refrigerated for further used. One $\mathrm{mL}$ of each stock solution (and $0.5 \mathrm{~mL}$ of the vitamin stock) was added to $1 \mathrm{~L}$ Milli-Q ultrapure water. The final COMBO medium was kept refrigerated for further use and warmed up to room temperature before inoculating with rotifer and algae mixtures.

\subsection{Statistical analysis of data}

All data were analyzed statistically with Systat software version 13. Sets of results were analyzed for parametric assumption. Each parameter, separated by behaviour and incubation time, was tested individually using a Kolmogorov-Smirnov Test $(p>0.05)$ and an equality of variances test, i.e. Bartlett's and Levene's ( $p>0.02)$. If parametric assumptions were met as described above, parameters were analyzed using Tukey's Honestly-Significant-Difference ANOVA. If the data did not meet parametric assumptions (with p-values smaller than the values stated above), they were analyzed non-parametrically using Kruskal-Wallis one-way ANOVA. 


\subsection{RESULTS AND DISCUSSION}

The results of experiments examining Euglena gracilis behaviour under stress of atrazine, TBT, and copper examined in this study are discussed in this section. The different methods of assessing behavioural changes in E. gracilis are assessed, including the suitability of the image analysis system ECOTOX for use in an early warning system for drinking water quality. The suitability and usability of the DaphniaTox image analysis system is also discussed, as well as the suitability of rotifers as indicator species and test organisms. Conclusions and recommendations are made based on findings discussed.

\subsection{Euglena gracilis motility - visual observation}

Cell motility of Euglena gracilis presents different sensitivities for the various contaminants tested. This is a positive trait when considering E. gracilis as an indicator organism because of its discriminating properties. Motility itself may be a discriminating parameter, as other behavioural parameters of E. gracilis may exhibit different sensitivities to contaminants. Cell motility was measured via visual observation, and as such it may be susceptible to human error and observer bias. However, the counting method is relatively fast and the data were consistent across replicates, suggesting that this is a reliable method for assessing behavioural changes in E. gracilis.

\subsubsection{Euglena gracilis motility after atrazine exposure}

Cell motility of E. gracilis was observed after $1 \mathrm{~h}, 2 \mathrm{~h}$, and $24 \mathrm{~h}$ exposure to atrazine at concentrations of $23.2 \mathrm{nM}(5 \mu \mathrm{g} / \mathrm{L}), 231.8 \mathrm{nM}(50 \mu \mathrm{g} / \mathrm{L})$, and $2318.2 \mathrm{nM}(500$ $\mu \mathrm{g} / \mathrm{L}$ ) in $0.1 \%$ DMSO, as well as $0.1 \%$ DMSO alone. Cell motility in $0.1 \%$ DMSO did not differ from cell motility in reference conditions. Atrazine treatment conditions did not differ from reference conditions throughout the experimentation period, including after 
24h. Results are summarized in Figure 3.1. Results were interpreted statistically using ANOVA (Tukey's test) after testing for parametric assumption.

These results are consistent with findings by Lockert et al. (2006), who examined growth of different algae taxa (green algae, diatoms, cryptomonad, cyanobacteria, and Euglenoid E. gracilis) after 5 days at $1000 \mu \mathrm{g} / \mathrm{L}$, twice the concentration of this experiment, and found E. gracilis to be the least sensitive species tested, with no growth inhibition seen.

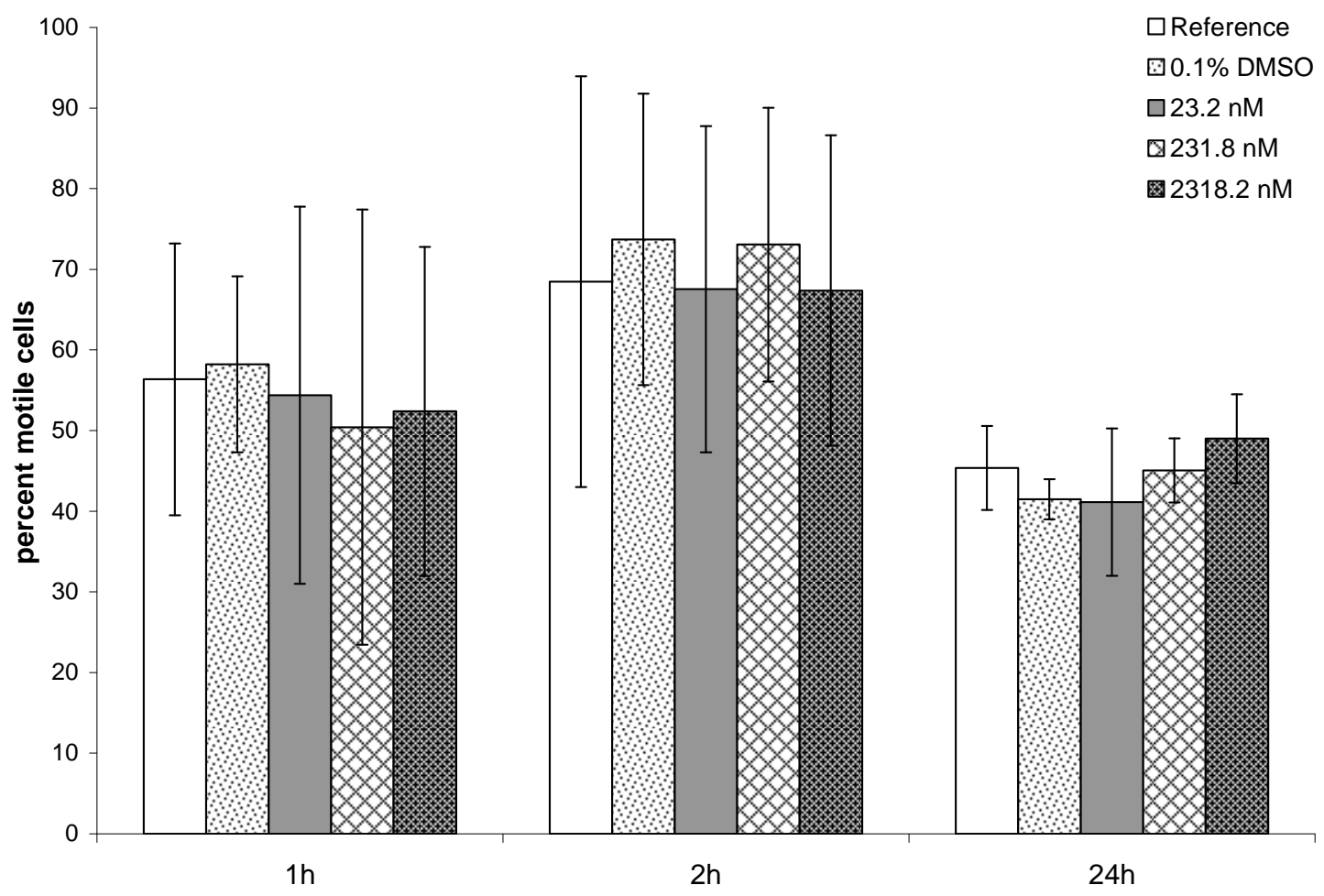

Figure 3.1. Average cell motility (visual observation) of $E$. gracilis after $1 \mathrm{~h}, 2 \mathrm{~h}$, and $24 \mathrm{~h}$ exposure to atrazine, showing no significant difference between treatments. Bars indicate standard deviation. 


\subsubsection{Euglena gracilis motility after tributyltin exposure}

Cell motility of E. gracilis was observed after $1 \mathrm{~h}, 2 \mathrm{~h}$, and $24 \mathrm{~h}$ exposure to a range of TBT concentrations, as well as DMSO alone (0.1\%). Cell motility in $0.1 \%$ DMSO did not differ from cell motility in reference conditions. None of the treatments were statistically different from reference conditions at any of the incubation times examined. Results are shown in Figure 3.2. After 24h there is an effect in E. gracilis motility in comparison with the $0.1 \%$ DMSO reference treatment at $10 \mu \mathrm{g} / \mathrm{L}(\mathrm{p}=0.027)$ and 100 $\mu \mathrm{g} / \mathrm{L}(\mathrm{p}=0.022)$, but not in comparison to reference conditions without DMSO. Results were interpreted statistically using ANOVA (Tukey's test) after testing for parametric assumption.

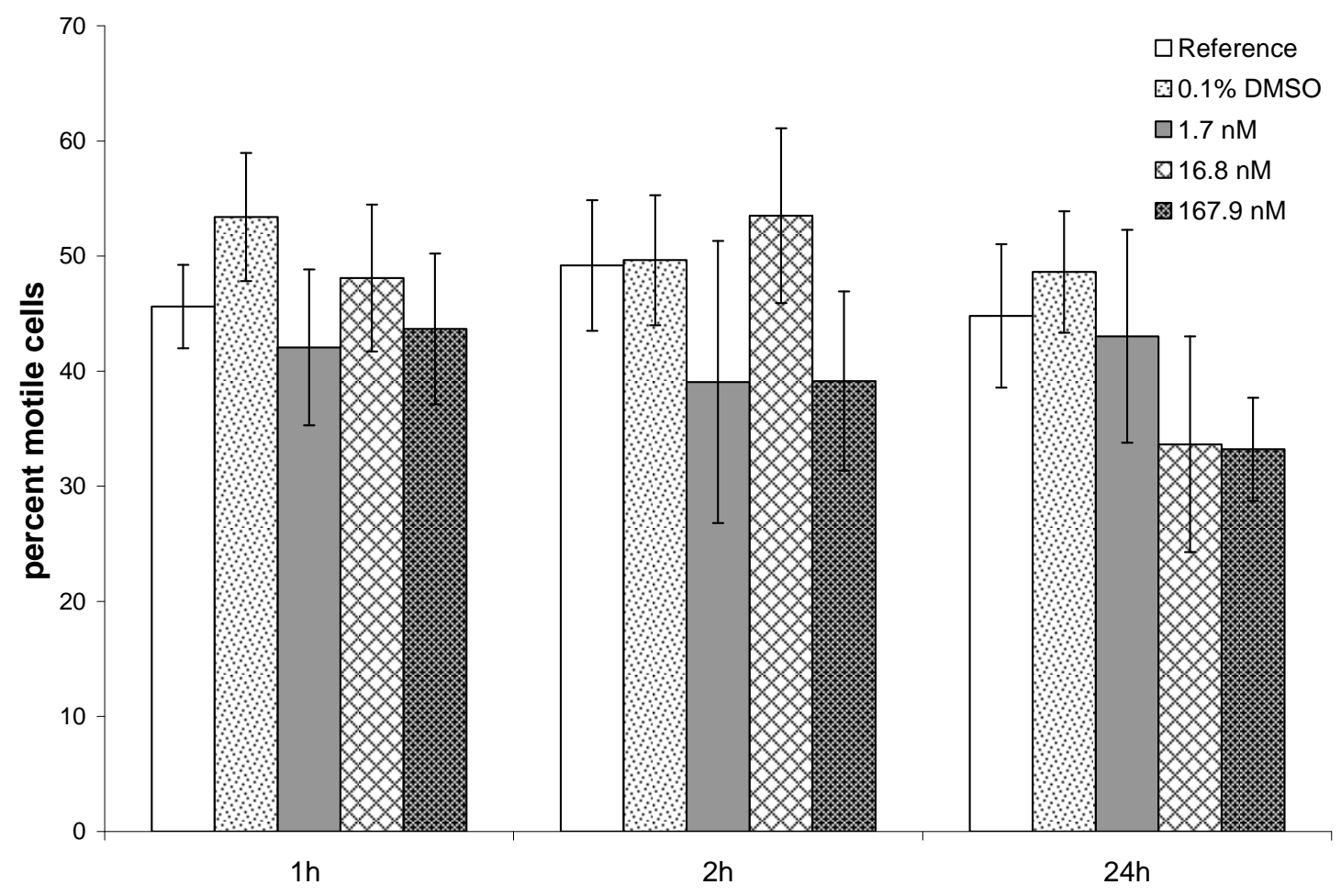

Figure 3.2. Average cell motility (visual observation) of $E$. gracilis after $1 \mathrm{~h}, 2 \mathrm{~h}$, and $24 \mathrm{~h}$ exposure to tributyltin, showing no significant difference between treatments. Bars indicate standard deviation. 
E. gracilis Z has been previously found to be affected by TBT. Ohta et al. (2007) observed cell motility at $50 \mu \mathrm{M}(29.8 \mathrm{mg} / \mathrm{L})$ of TBT and found cell immobilization after 5 minutes exposure. This concentration is an order of magnification higher than concentrations used for this experiment, and incubation time was much shorter at 5 minutes in comparison with $1 \mathrm{~h}, 2 \mathrm{~h}$, and $24 \mathrm{~h}$ used in this experiment. It is likely that at higher concentration a motility effect would be seen, but it is not necessary that concentrations be as high as the ones found in Ohta et al. (2007) with longer incubation times.

\subsubsection{Euglena gracilis motility after copper exposure}

Cell motility of E. gracilis was observed after $1 \mathrm{~h}, 2 \mathrm{~h}$, and $24 \mathrm{~h}$ exposure to a range of concentrations of copper (added as $\mathrm{CuSO}_{4} \cdot 5 \mathrm{H}_{2} \mathrm{O}$ ), and was found to be very sensitive. Results were analyzed statistically with Kruskal-Wallis tests and are summarized in Figure 3.3. After $1 \mathrm{~h}$ and $2 \mathrm{~h}$, there were no changes in motility for concentrations of up to $0.4 \mu \mathrm{M}$, and a sharp drop in motility at $2.0 \mu \mathrm{M}$, averaging at $7.3 \%$ after $1 \mathrm{~h}(\mathrm{p}=0.004)$ compared to $45.9 \%$ and $45.1 \%$ motile cells in reference conditions and $0.4 \mu \mathrm{M}$ respectively. This lowered motility continued after $2 \mathrm{~h}$ incubation, where $8.5 \%$ cells were motile at $2.0 \mu \mathrm{M}(\mathrm{p}=0.004)$ compared to $47.8 \%$ motile cells at reference conditions and $47.5 \%$ in $0.4 \mu \mathrm{M}$. A $20 \mu \mathrm{M}$ treatment was added as a positive control, and under this treatment cell motility was found to be almost entirely inhibited, at $0.75 \%$ after $1 \mathrm{~h}(\mathrm{p}=0.004)$ and $1.54 \%$ after $2 \mathrm{~h}(\mathrm{p}=0.004)$.

After $24 \mathrm{~h}$ incubation, however, cells seem to recover and there is no longer a difference between treatments and reference conditions, even at the positive control concentration used, $20 \mu \mathrm{M}$, which reduced motility by nearly $100 \%$ at $1 \mathrm{~h}$ and $2 \mathrm{~h}$ (Figure 3.3). This seems to indicate that E. gracilis possesses a cellular mechanism for dealing with higher sublethal concentrations of copper but which takes effect between $2 \mathrm{~h}$ and $24 \mathrm{~h}$ after the start of the exposure period. One such mechanism exists in blue-green algae. 


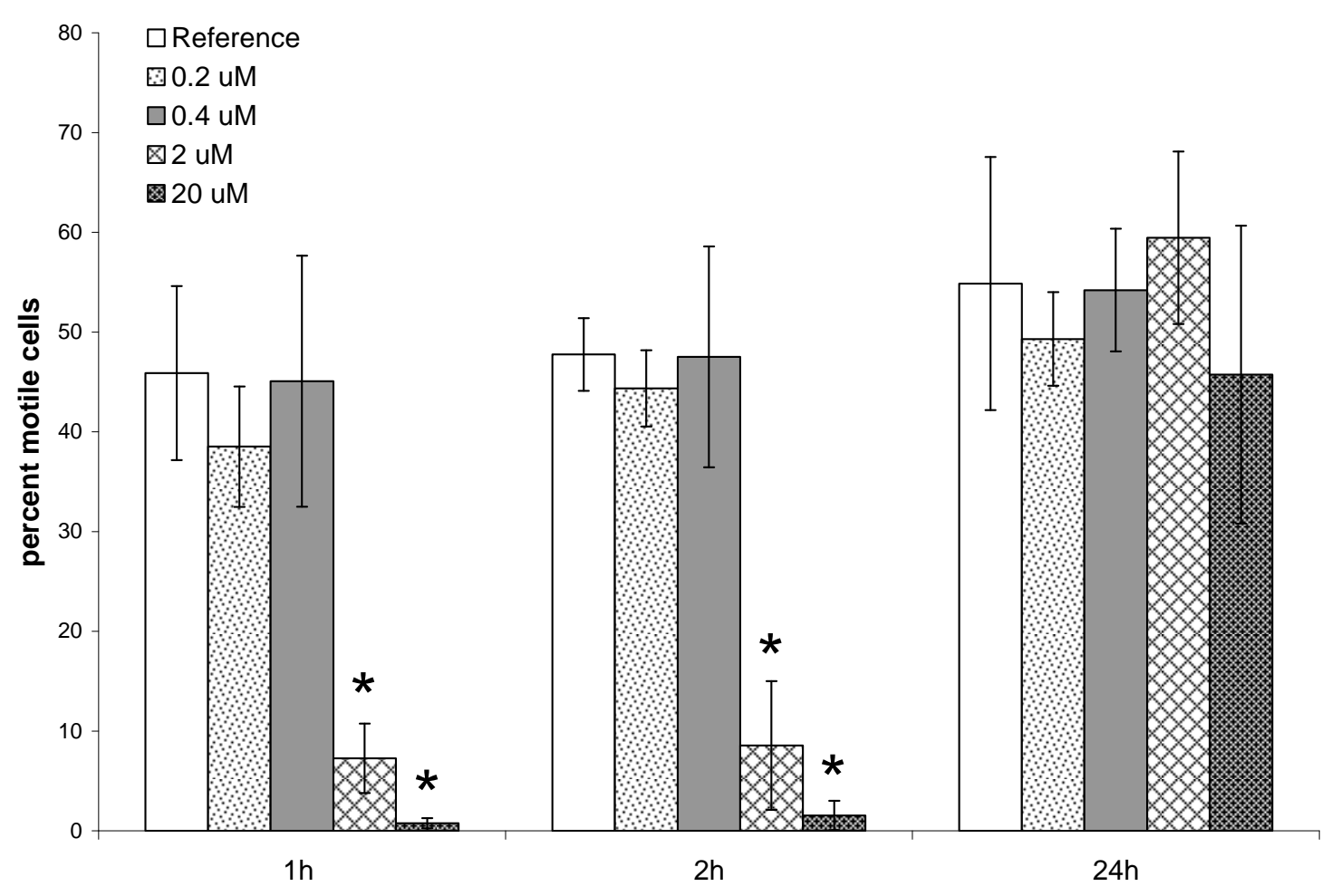

Figure 3.3. Average cell motility (visual observation) of $E$. gracilis after $1 \mathrm{~h}, 2 \mathrm{~h}$, and $24 \mathrm{~h}$ exposure to copper. Asterisks indicate a statistically significant difference from reference conditions. Bars indicate standard deviation.

Whitton (1965) found that an extracellular peptide produced by the blue-green alga Archaeoellipsoides cylindrica has the ability to chelate copper and reduce its toxicity to this species. In another study, Ohta and Suzuki (2007) mention that cell recovery was observed in E. gracilis after exposure to TBT by metabolizing the contaminant into a less toxic form and excreting it from the cell. Similar processes, or a combination of processes, may be occurring with E. gracilis after copper exposure, and further studies are needed to investigate the mechanism of action for this recovery of motility after longer periods of incubation. A recent study by Ahmed and Häder (2010) has also found that the cell motility $\mathrm{EC}_{50}$ of $E$. gracilis under copper stress is somewhat higher after $24 \mathrm{~h}$ than after 5 minutes ( $368.2 \mu \mathrm{M}$ after $24 \mathrm{~h}$ compared to $300.4 \mu \mathrm{M}$ ), suggesting this is a real observable phenomenon. Both of these concentrations are much greater than the ones used in this study. It is also important to note that acute toxicity tests on E. gracilis 
generally mean having examined parameters such as motility and cell shape after $24 \mathrm{~h}$ incubation in contaminant (Danilov \& Ekelund, 2001a, 2001b; Einicker-Lamas et al., 2002; Ahmed \& Häder, 2010a, 2010b). These results show that 24h tests are not showing the entire picture for the pattern of toxicity for copper, and potentially other contaminants as well; and, in fact, E. gracilis is very well suited for an early warning system, as its rapid responses are stronger at $1 \mathrm{~h}$ and $2 \mathrm{~h}$ than the traditional $24 \mathrm{~h}$ acute toxicity tests.

\subsection{3.a Effects of temperature on copper toxicity in Euglena gracilis}

The motility experiment described above was then expanded within the concentration range between $0.4 \mu \mathrm{M}$ and $2 \mu \mathrm{M}$ with the purpose of obtaining $\mathrm{EC}_{50}$ values for copper motility. The experiment was performed in the temperature range between 20 and $21^{\circ} \mathrm{C}$, as well as in the temperature range between 25 and $26^{\circ} \mathrm{C}$. Figure 3.4 shows a trend of increasing sensitivities at higher temperatures. Patterns of motility inhibition at increasing concentrations were similar at $1 \mathrm{~h}$ an $2 \mathrm{~h}$. At $21^{\circ} \mathrm{C}$, motility differed significantly from reference conditions for concentrations above $1.2 \mu \mathrm{M}(\mathrm{p}=0.012$, 0.001 , and $<0.001$ for $1.2,1.6$, and $2 \mu \mathrm{M}$ respectively after $1 \mathrm{~h}$, and $\mathrm{p}=0.026,0.014$, and $<0.01$ for $1.2,1.6$, and $2 \mu \mathrm{M}$ respectively after $2 \mathrm{~h}$ ). At $26^{\circ} \mathrm{C}$, a $5^{\circ} \mathrm{C}$ increase in temperature from the previous test, E. gracilis showed a response in increased inhibition of motility at lower concentrations. Motility was inhibited at concentrations of $0.8 \mu \mathrm{M}$ and above $(\mathrm{p}=0.009$ for all concentrations $0.8 \mu \mathrm{M}$ and above after $1 \mathrm{~h}$ and $2 \mathrm{~h}$ incubation).

The $\mathrm{EC}_{50}$ value for motility after copper exposure was calculated from the doseresponse relationships at different temperatures using ED50plus v1.0, created by M. H. Vargas, Mexico, 2000. The $\mathrm{EC}_{50 \mathrm{~s}}$ at $21^{\circ} \mathrm{C}$ was $1.389 \mu \mathrm{M}$ copper after $1 \mathrm{~h}$ and $1.529 \mu \mathrm{M}$ copper after $2 \mathrm{~h}$. The $\mathrm{EC}_{50 \mathrm{~s}}$ calculated for the tests at $26^{\circ} \mathrm{C}$ were lower, at $0.947 \mu \mathrm{M}$ after $1 \mathrm{~h}$ and $0.818 \mu \mathrm{M}$ after $2 \mathrm{~h}$. This represents a $46 \%$ decrease in the $\mathrm{EC}_{50}$ after $2 \mathrm{~h}$

incubation, and indicates that E. gracilis is more sensitive to copper at higher temperatures. 


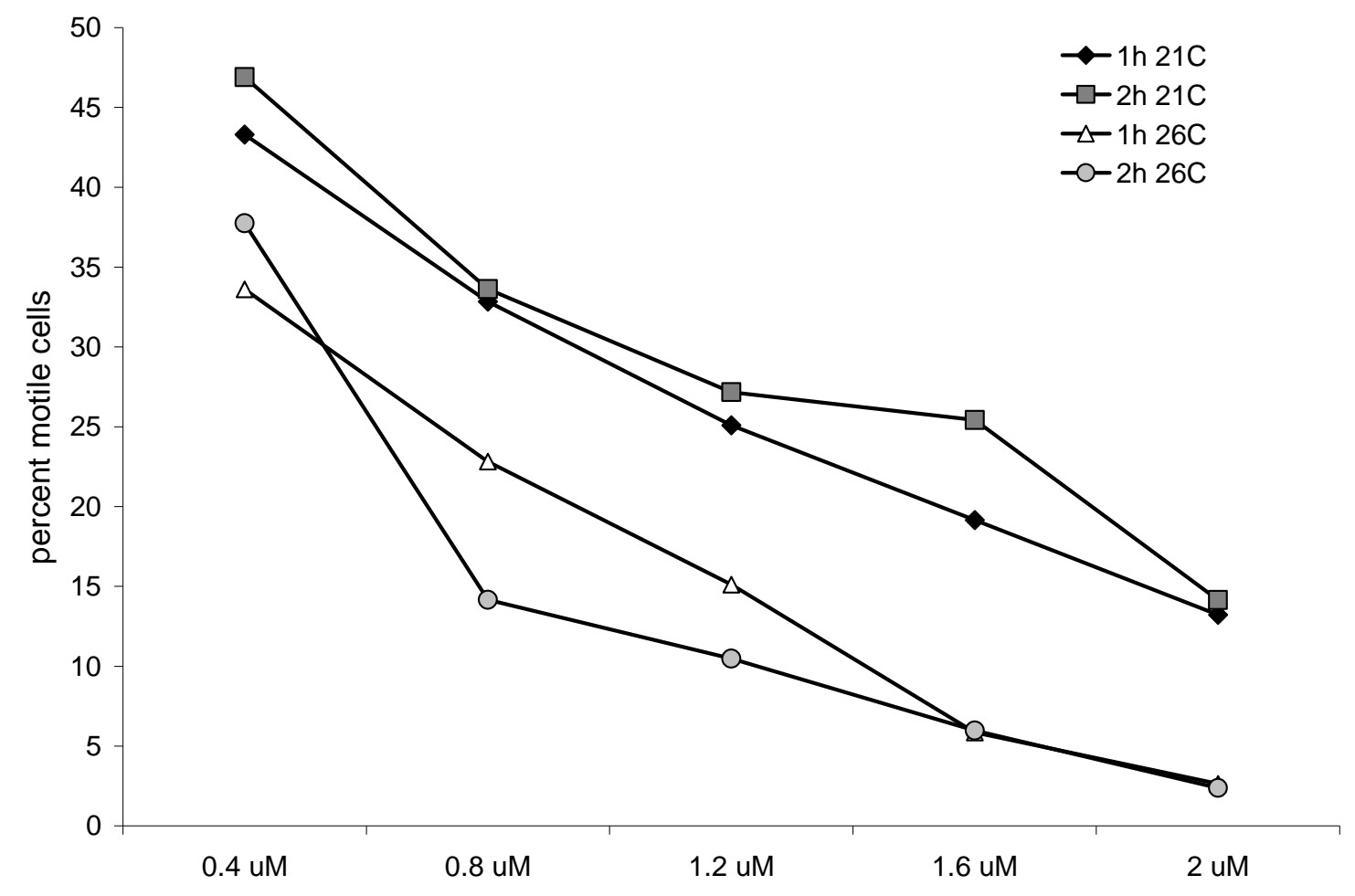

Figure 3.4. Cell motility of $E$. gracilis after $1 \mathrm{~h}$, and $2 \mathrm{~h}$ exposure to copper at $21^{\circ} \mathrm{C}$ and $26^{\circ} \mathrm{C}$. The $\mathrm{EC}_{50 \mathrm{~s}}$ at $21^{\circ} \mathrm{C}$ was $1.389 \mu \mathrm{M}$ after $1 \mathrm{~h}$ and $1.529 \mu \mathrm{M}$ after $2 \mathrm{~h}$. The $\mathrm{EC}_{50 \mathrm{~s}}$ at $26^{\circ} \mathrm{C}$ were $0.947 \mu \mathrm{M}$ after $1 \mathrm{~h}$ and $0.818 \mu \mathrm{M}$ after $2 \mathrm{~h}$.

These results were expected in that the effects of temperature on metal toxicity have been studied before, where it was suggested that it may act by generally increasing the metabolism of cells, and particularly for aquatic organisms a rise of $10^{\circ} \mathrm{C}$ in temperature will roughly double the metabolic rate (Cairns et al., 1975). The implication of this for research is that experiments with E. gracilis must be carefully temperature-controlled. A standard must also exist to make testing with E. gracilis consistent at a specific temperature, as variations in testing temperature may have a large effect on copper toxicity and perhaps other contaminants as well. It may also be desirable to increase testing temperatures so as to purposely increase the sensitivity of this organism. 


\subsection{3.b. Effects of water hardness on copper toxicity in Euglena gracilis}

Several physicochemical parameters other than temperature also influence the toxicity and bioavailability of copper and other heavy metals to aquatic organisms, such as pH, alkalinity, DOC, and especially water hardness (Erickson et al., 1996). The effects of water hardness on copper toxicity to aquatic organisms have been extensively studied, and it has been determined that increasing water hardness reduces the toxicity of copper (Erickson et al., 1996; Welsch et al., 2000; Ryan et al., 2009). One mode of action suggested is that copper directly competes with calcium in binding sites in the cell (Erickson et al., 1996). Experiments with E. gracilis were carried out in mineral medium, with a total hardness of $94.4 \mathrm{ppm}$ (Appendix B). This is very close to the range of water hardness found in municipal tap water in Toronto, between 106 and 127 ppm (City of Toronto, 2010) and therefore this medium was deemed appropriate to test for copper toxicity in the context of a drinking water quality testing system.

However, studies have determined that water hardness does not provide sufficient information for predicting variations of the toxicity of copper. In fact, calcium and magnesium cations have been found to have very different effects in the copper toxicity of several species of freshwater fish, where increasing concentrations of $\mathrm{Ca}^{2+}$ decreased copper toxicity linearly but increasing $\mathrm{Mg}^{2+}$ concentrations did not (Welsch et al., 2000). Despite this, de Schamphelaere and Janssen (2002) investigated the effects of calcium and magnesium cations on the toxicity of copper to Daphnia magna and found that both of these cations decreased copper toxicity to a similar extent. Given that magnesium contributes to $85 \%$ of the hardness of the E. gracilis mineral medium, and the proportion of calcium to magnesium cations in municipal tap water in Toronto is unknown, it is important for future studies to investigate whether the toxicity of copper for E. gracilis is affected by both calcium and magnesium cations equally, as this may lead to false negatives, failing thus to accurately monitor the presence of a heavy metal contamination. Of equal concern is to investigate whether the toxicity of copper in mineral medium is equivalent to the toxicity of copper in the drinking water to be tested, especially when these toxicity tests are used to determine drinking water quality. 


\subsection{Euglena gracilis cell shape - visual observation}

The cell shapes of Euglena gracilis were observed after incubation in copper (0.4 $\mu \mathrm{M}, 1.2 \mu \mathrm{M}, 2 \mu \mathrm{M}$, and $20 \mu \mathrm{M}$ ) for $1 \mathrm{~h}$ and $2 \mathrm{~h}$. Previous research at Ryerson University by C. Pearce (2009) has found that E. gracilis cell shape changes from elongated to round after $6 \mathrm{~h}$ exposure to $10 \mu \mathrm{g} / \mathrm{L}$ of TBT and $50 \mu \mathrm{g} / \mathrm{L}$ of atrazine. Due to the rapid response times of E. gracilis motility to copper stress, cell shape experiments were performed at $1 \mathrm{~h}$ and $2 \mathrm{~h}$ incubation in copper. The assigned values for cell shape were 0 for round/cyst form, 1 for oval, and 2 for elongated shape. Results of cell shape tests were similar after $1 \mathrm{~h}$ and $2 \mathrm{~h}$. Differences in average cell shape were more marked after 1h (Figure 3.5), where average cell shape was $1.53 \pm 0.34$ at reference conditions, $1.00 \pm 0.14$ at $2 \mu \mathrm{M}$ copper and $0.56 \pm 0.14$ at $20 \mu \mathrm{M}$ copper, corresponding to $35 \%$ and $63 \%$ inhibition respectively. After $2 \mathrm{~h}$ incubation, average cell shape was $1.64 \pm 0.10$ at reference conditions, $1.25 \pm 0.13$ at $2 \mu \mathrm{M}$ copper and $1.00 \pm 0.14$ at $20 \mu \mathrm{M}$ copper, corresponding to $24 \%$ and $39 \%$ inhibition respectively. However, statistical analysis using ANOVA (Tukey's test) has shown a significant difference after $1 \mathrm{~h}$ incubation for $20 \mu \mathrm{M}$ only, whereas after $2 \mathrm{~h}$ incubation both the $2 \mu \mathrm{M}$ and the $20 \mu \mathrm{M}$ treatments were found to be significantly different from reference conditions. This is due to the high variability in the results for cell shape at reference conditions after $1 \mathrm{~h}$, which may suggest that these cells are still in an adaptation period after the physical stress of being transferred from the culture flask to the experimental vials. This idea is strengthened by the fact that average cell shape is more elongated in reference conditions after $2 \mathrm{~h}(1.64 \pm 0.10)$ than after $1 \mathrm{~h}$ incubation $(1.53 \pm 0.34)$.

Despite an obvious trend in cell shapes becoming rounder at higher concentrations of copper, results were only significant for concentrations above $20 \mu \mathrm{M}$ after $1 \mathrm{~h}$ incubation and for concentrations above $2 \mu \mathrm{M}$ after $2 \mathrm{~h}$ incubation. In contrast, in the results from the motility experiment, a significant difference was found for concentrations above $1.2 \mu \mathrm{M}$ at $21^{\circ} \mathrm{C}$ after $1 \mathrm{~h}$ and $2 \mathrm{~h}$ incubation, and for concentrations above $0.8 \mu \mathrm{M}$ at $26^{\circ} \mathrm{C}$ after $1 \mathrm{~h}$ and $2 \mathrm{~h}$ incubation, showing that cell motility is a more sensitive parameter for indicating copper toxicity in E. gracilis than cell shape. Despite this, cell shape may still be a suitable parameter as a water quality indicator, since 
experiments with the organic contaminants atrazine and TBT did not show a difference in cell motility even after $24 \mathrm{~h}$ in comparison to reference, whereas a previous study by C. Pearce (2009) at Ryerson University has found a difference in E. gracilis cell shape after $6 \mathrm{~h}$ atrazine and TBT exposure.

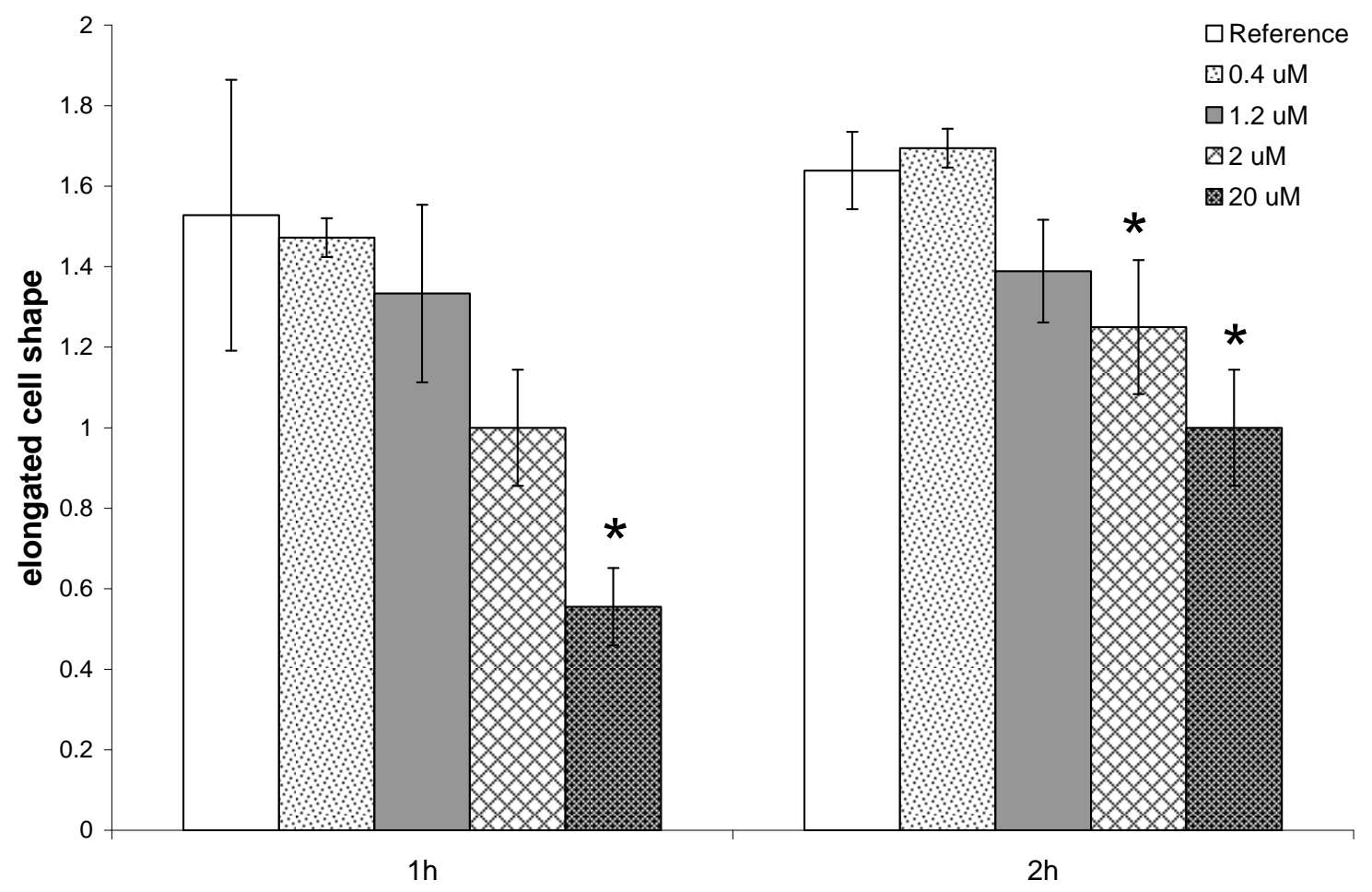

Figure 3.5. Euglena gracilis cell shape under copper stress after $1 \mathrm{~h}$ and $2 \mathrm{~h}$ incubation. Asterisks indicate results that are significantly different from reference conditions. Assigned values for cell shape are as follows: 2 for elongated, 1 for oval, and 0 for round. Cyst formation is a stress response as cells become rounder at increasing concentrations of copper.

\subsection{Euglena gracilis automated parameters measured with ECOTOX}

Euglena gracilis behaviour was assessed using the image analysis system ECOTOX to assess whether this automated system is suitable for inclusion in an early warning system for drinking water quality. The behavioural parameters analyzed were 
cell swimming velocity (given in $\mu \mathrm{m} / \mathrm{s}$ ), cell shape (given in form factor, i.e. a ratio of circumference to area), randomness of swimming (given in r-value), and gravitactic behaviour (given in percent cells swimming up). The same three contaminants used for the cell motility experiment (atrazine, TBT, and copper) were tested using the automated system parameters, and results are shown below. The parameters were then assessed individually to determine their sensitivity and reliability for inclusion in the miner's canary system. All parameters were analyzed statistically using ANOVA (Tukey's test), except for cell swimming velocity after exposure to atrazine and TBT, which were analyzed using nonparametric Kruskal-Wallis tests after testing all parameters for parametric assumption.

\subsubsection{Euglena gracilis automated parameters after atrazine exposure}

After $2 \mathrm{~h}$ and $24 \mathrm{~h}$ incubation in atrazine concentrations of 23.2, 231.8, and 2318.2 $\mathrm{nM}$ (corresponding to 5, 50, and $500 \mu \mathrm{g} / \mathrm{L}$ respectively), the behaviour of Euglena gracilis was analyzed using the ECOTOX system. The results are summarized in Figures 3.6 through 3.9 .

\subsection{1.a. Gravitactic behaviour and atrazine exposure}

After examining cell velocity, shape, and swimming randomness, it was found that these parameters did not differ from reference conditions after either $2 \mathrm{~h}$ or $24 \mathrm{~h}$ incubation time for any of the atrazine concentrations. There were significant changes from reference conditions; however, in the gravitactic behaviour (Figure 3.7) after 24h incubation in atrazine for concentrations of $231.8 \mathrm{nM}(\mathrm{p}=0.003)$ and $2318.2 \mathrm{nM}(\mathrm{p}=$ 0.005). In contrast with the observational method of assessing cell motility, the automated system was more sensitive at detecting differences in cell movement, as motility observations did not yield any significant results. It is expected that visual observations are unable to detect small changes in cell swimming velocity. 


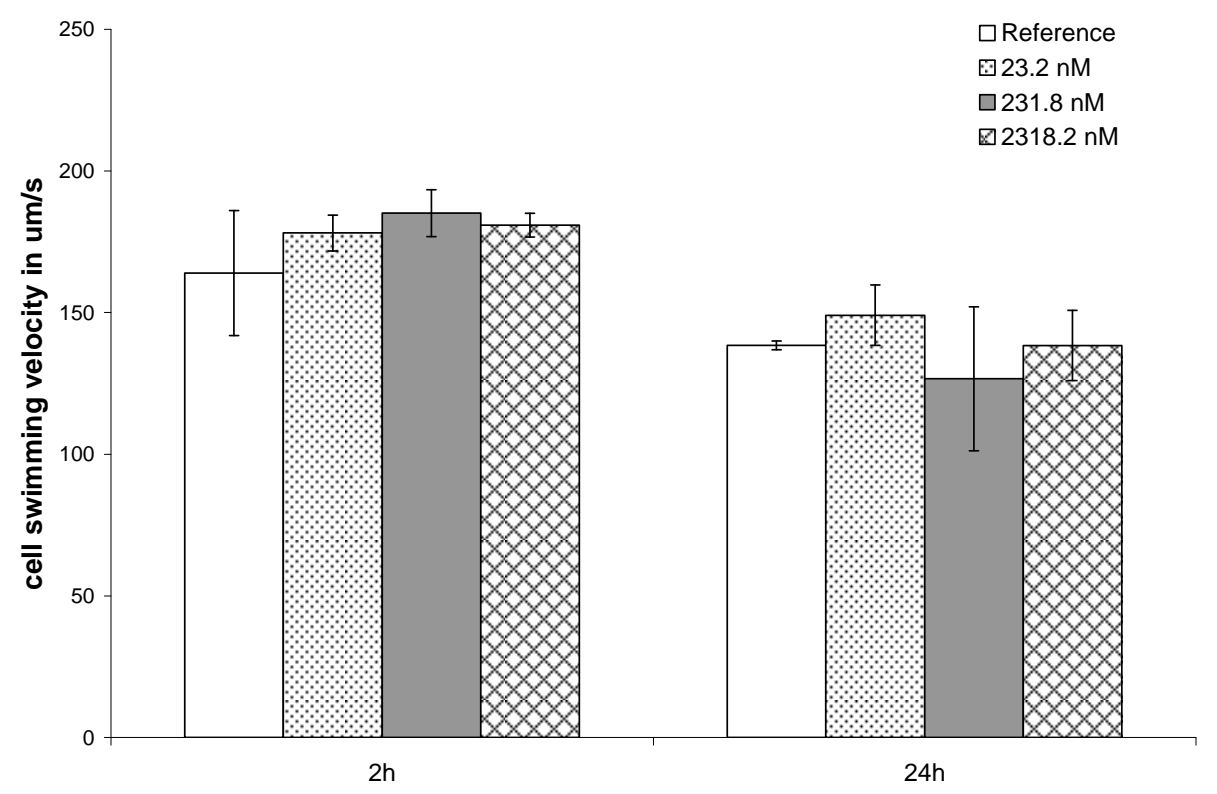

Figure 3.6. Euglena gracilis cell velocity after incubation in atrazine in $23.2,231.8$, and $2318.2 \mathrm{nM}$. No significant differences from reference were found. Bars indicate standard deviation.

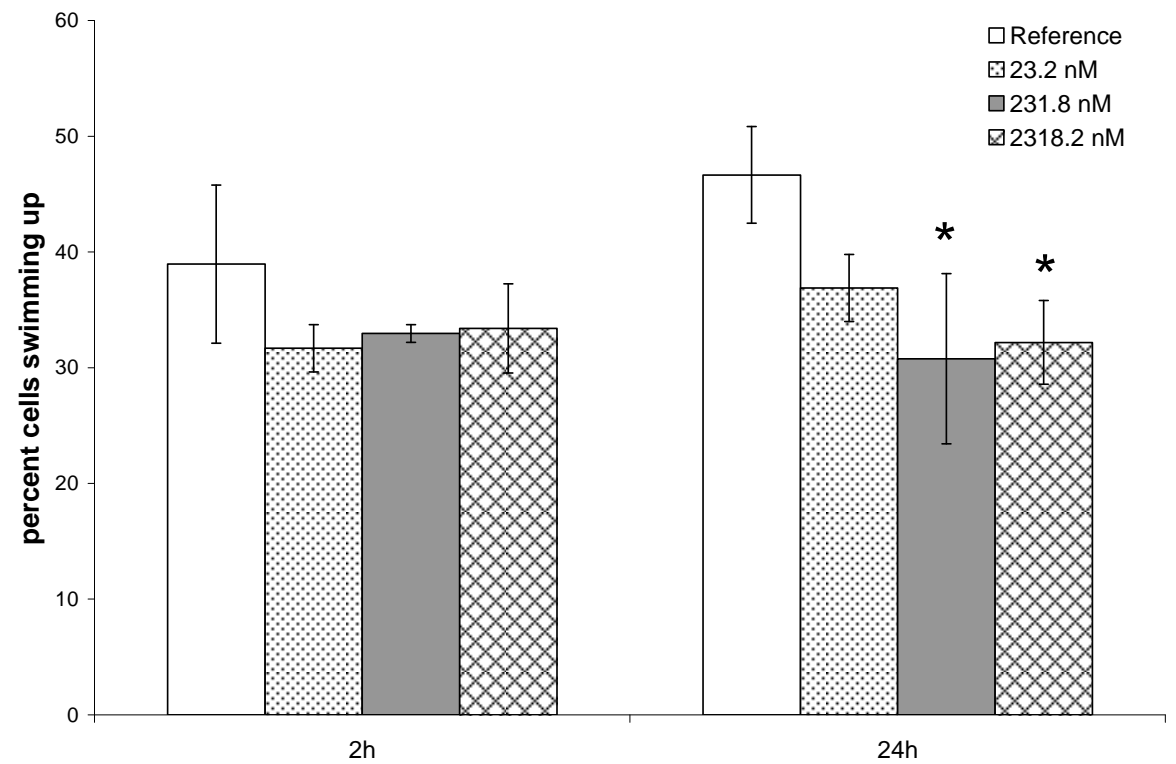

Figure 3.7. Euglena gracilis gravitactic orientation after incubation in atrazine in 23.2, 231.8, and $2318.2 \mathrm{nM}$. After 24h incubation, the number of cells swimming up was significantly reduced. Asterisks indicate results significantly different from reference. Bars indicate standard deviation. 


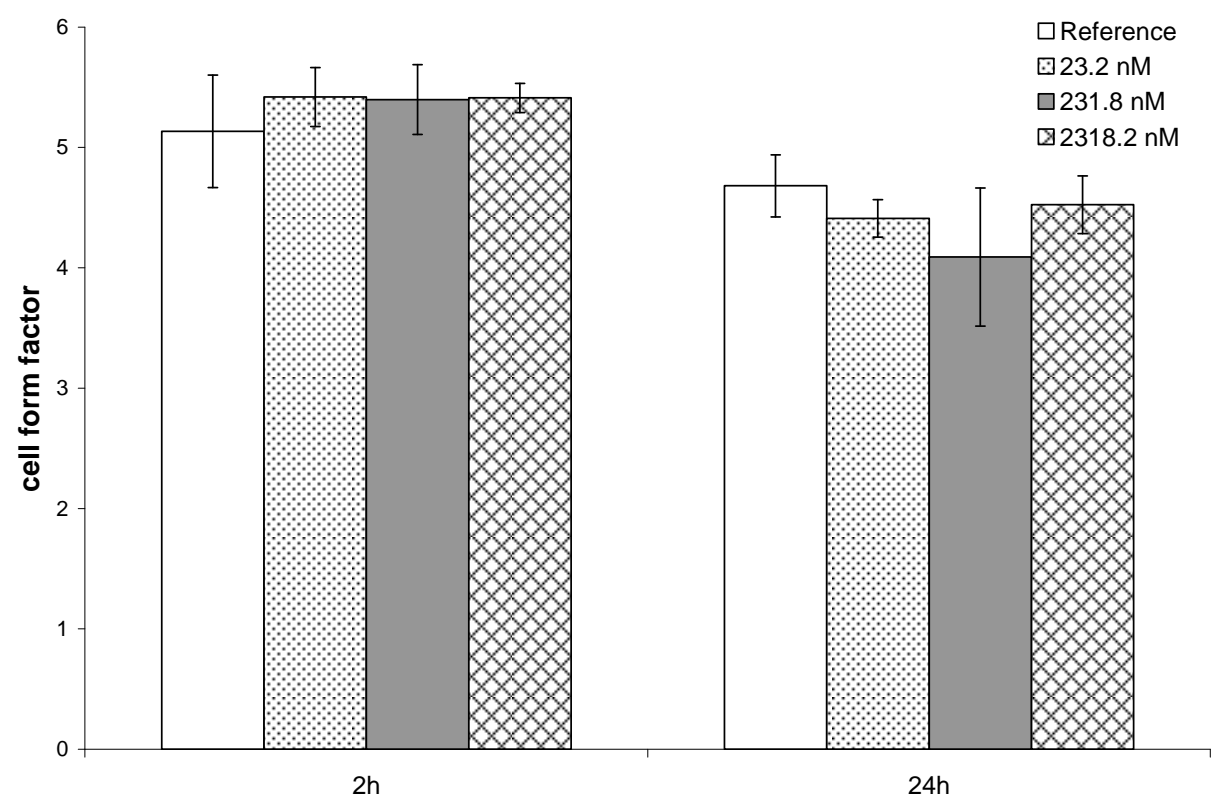

Figure 3.8. Euglena gracilis cell shape (decreasing numbers represent change towards cyst-form) after incubation in atrazine in 23.2, 231.8, and $2318.2 \mathrm{nM}$. No significant differences from reference were found. Bars indicate standard deviation.

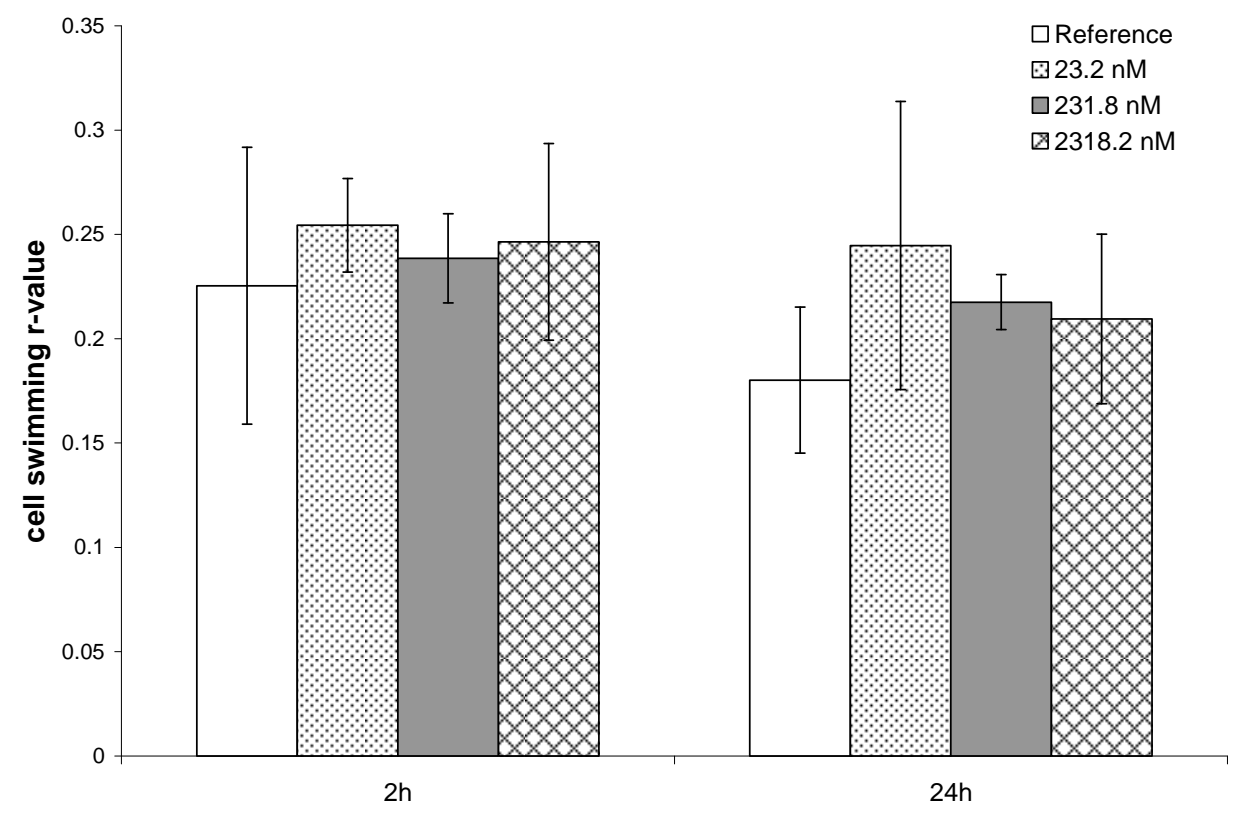

Figure 3.9. Euglena gracilis randomness of swimming (decreasing numbers represent more random swimming) after incubation in atrazine in 23.2, 231.8, and $2318.2 \mathrm{nM}$. No significant differences from reference were found. Bars indicate standard deviation. 
A previous study by Lockert et al. (2006) investigating the effects of atrazine on algae growth rates had found that unlike all the other freshwater species studied, which included green algae, cyanobacteria, diatoms, and a cryptomonad, E. gracilis growth was unaffected at concentrations as high as $1000 \mu \mathrm{g} / \mathrm{L}(4636.4 \mathrm{nM})$. This indicates that gravitactic behaviour in E. gracilis is a very sensitive endpoint for atrazine at low concentrations, when even much higher concentrations do not affect either its cell motility or growth rates.

\subsection{1.b. Velocity and atrazine exposure}

Another question to be addressed with automated image analysis parameters is whether the cell velocity parameter measured by ECOTOX is more sensitive, less sensitive, or comparable to visual observations of motility under the microscope. Since no significant results were produced by either visual observation (Figure 3.1) or by automated image analysis (Figure 3.6), it cannot be said whether one is more sensitive than the other; however, in the context of an early warning system, it can be said that cell swimming velocity cannot be used as an indicator of atrazine presence in water samples tested.

\subsection{1.c. Cell shape and atrazine exposure}

The automated analysis of cell shape equally yielded no significant results (Figure 3.8), when previous research using visual observation had found effects on E. gracilis cell shape after $6 \mathrm{~h}$ exposure to $231.8 \mathrm{nM}$ atrazine. This indicates that the automated system is less sensitive at detecting morphological changes in cell shape than visual observation. Because no changes were detected with the automated system's cell shape parameter after atrazine exposure at several concentrations, this parameter was considered not appropriate for detecting the presence of atrazine in an early warning system. 


\subsection{1.d. $R$-value and atrazine exposure}

Analysis of randomness of swimming also did not show any significant results (Figure 3.9). Because it is related to the percent number of cells swimming up, as when this number approaches $100 \%$ the r-value would too represent a non-random pattern of swimming (approaching r-value $=1$ ), it was expected that there would be a difference seen in r-values measured since there was a significant difference in percent number of cells swimming up. Further, in previous research published by the developers of ECOTOX, Tahedl and Häder (1999; 2001), the r-value was the most sensitive parameter for 11 out of 20 contaminants tested, whereas the percent number of cells swimming up was not found to be the most sensitive parameter for any of the contaminants tested, suggesting that $r$-value is in general a more sensitive parameter than number of cells swimming up. However, this was not seen in this case. In general, average r-values tended to be closer to random than to non-random patterns of swimming, ranging from 0.18 to 0.25 . There also tended to be a high degree of variance for this data. These results contradict previous results found by Tahedl and Häder (1999; 2001), and therefore further tests using this parameter are necessary.

\subsubsection{Euglena gracilis automated parameters after TBT exposure}

After $2 \mathrm{~h}$ and $24 \mathrm{~h}$ incubation in TBT concentrations of 1,10 , and $100 \mu \mathrm{g} / \mathrm{L}$, the behaviour of Euglena gracilis was analyzed using the ECOTOX system. The results are summarized in Figures 3.10 through 3.13.

\subsection{2.a. Gravitactic behaviour and TBT exposure}

When cell velocity, shape, and swimming randomness were examined, it was found that these parameters did not differ from reference conditions after either $2 \mathrm{~h}$ or $24 \mathrm{~h}$ incubation time for any of the atrazine concentrations. 


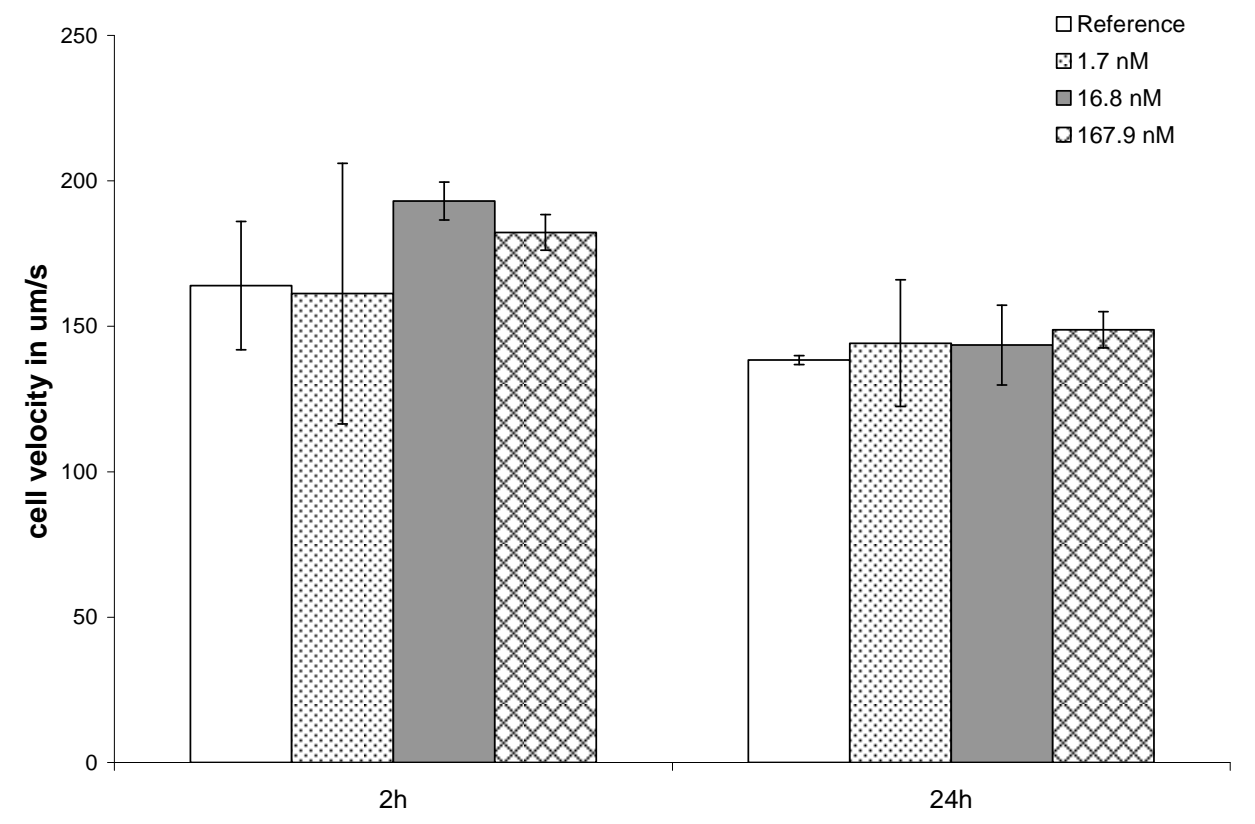

Figure 3.10. Euglena gracilis cell velocity after incubation in TBT in 1.7, 16.8, and $167.9 \mathrm{nM}$. No significant differences from reference were found. Bars indicate standard deviation.

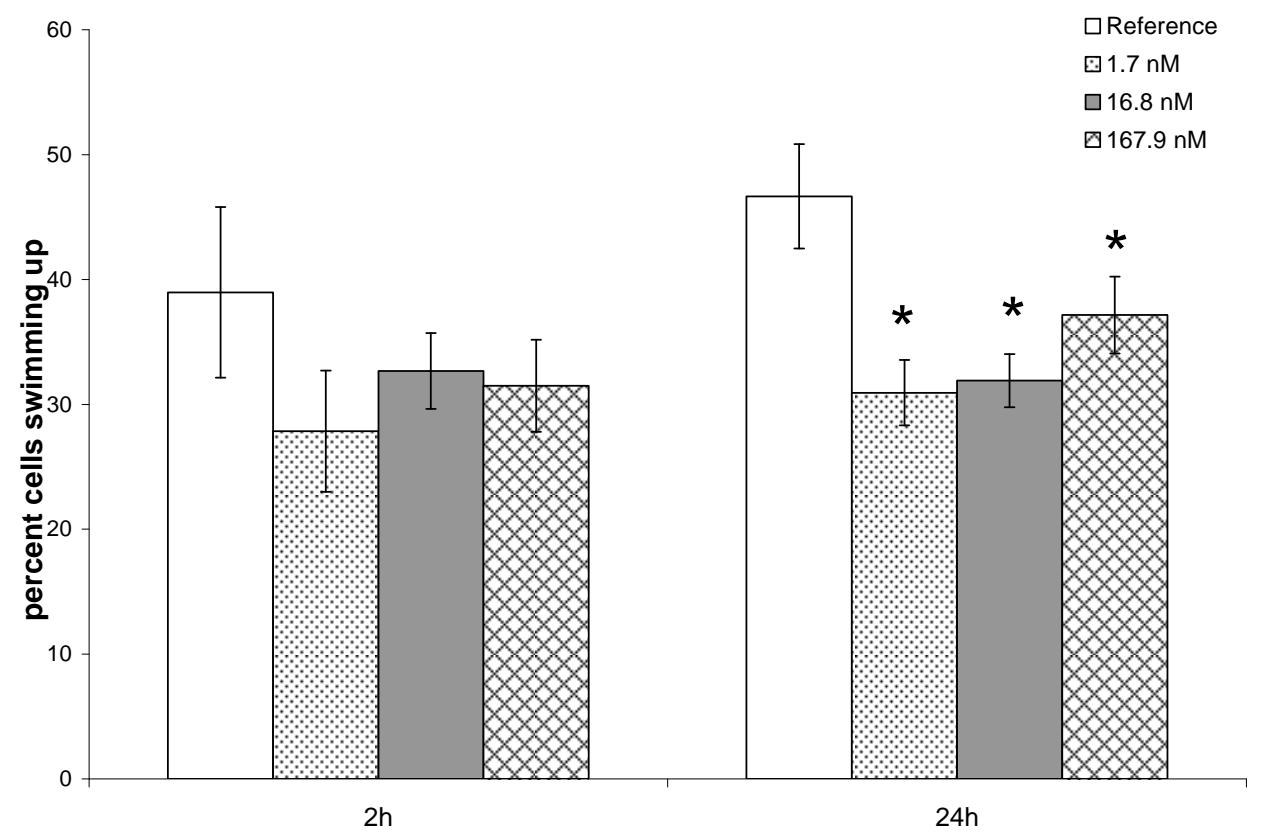

Figure 3.11. Euglena gracilis gravitactic orientation after incubation in TBT in 1.7, 16.8, and $167.9 \mathrm{nM}$. Asterisks indicate results that are significantly different from reference. Bars indicate standard deviation. 


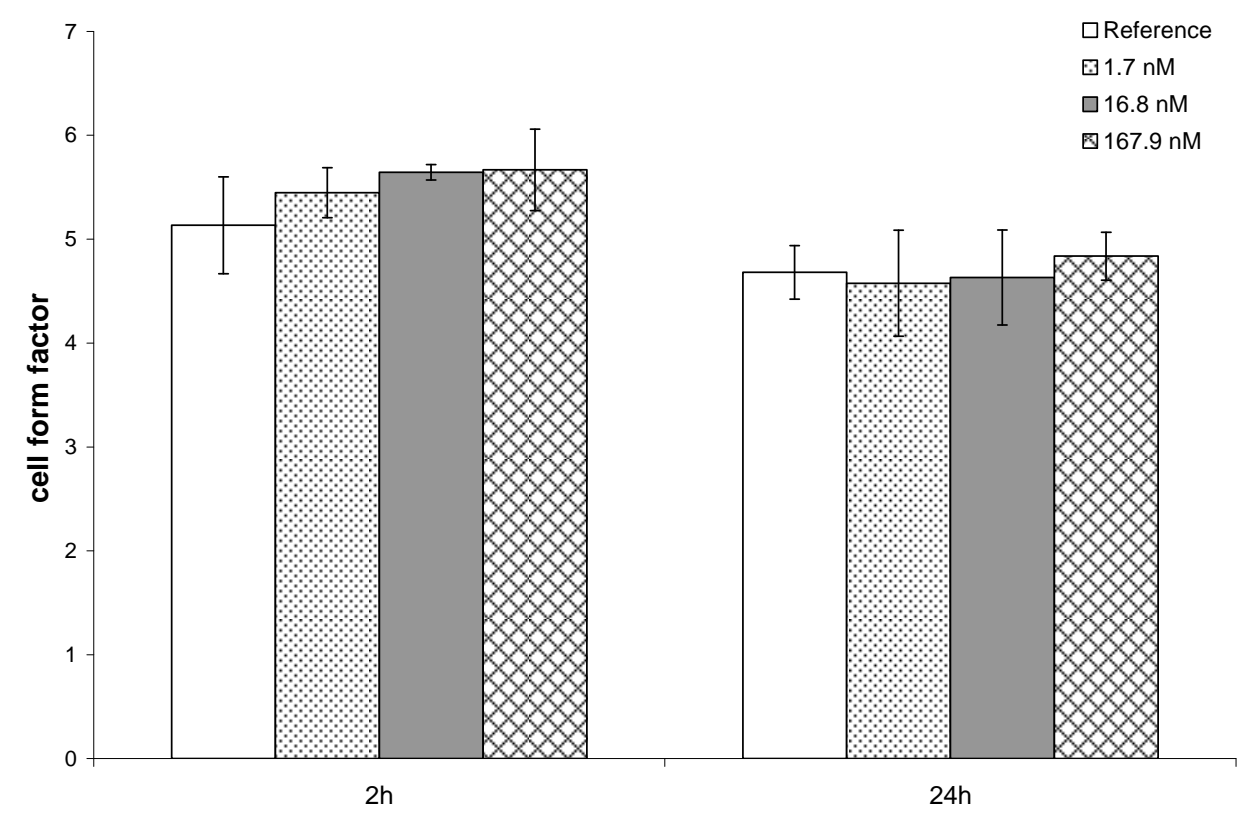

Figure 3.12. Euglena gracilis cell shape (decreasing numbers represent change towards cyst-form) after incubation in TBT in 1.7, 16.8, and $167.9 \mathrm{nM}$. No significant differences from reference were found. Bars indicate standard deviation.

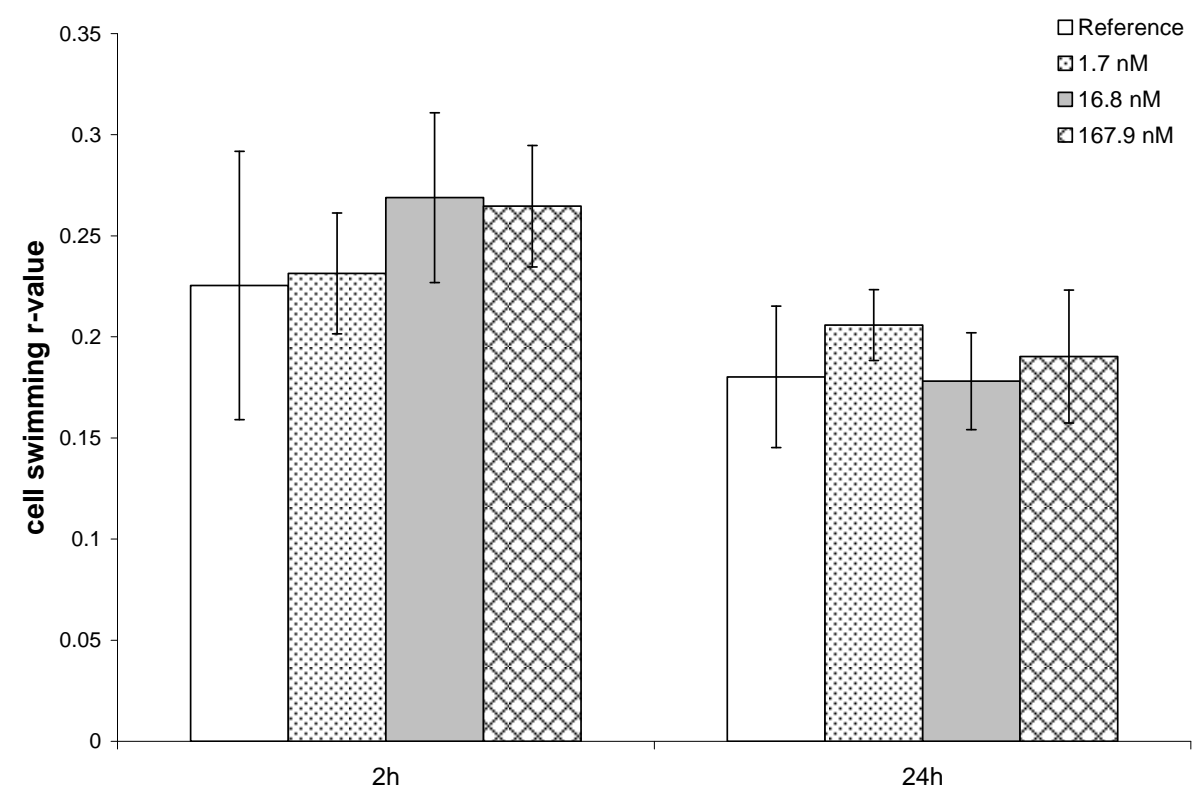

Figure 3.13. Euglena gracilis randomness of swimming (decreasing numbers represent more random swimming) after incubation in TBT in 1.7, 16.8, and $167.9 \mathrm{nM}$. No significant differences from reference were found. Bars indicate standard deviation. 
However, much like with atrazine exposure, there were significant changes in the gravitactic behaviour after $24 \mathrm{~h}$ incubation in TBT for all concentrations (Figure 3.11): 1 $\mu \mathrm{g} / \mathrm{L}(\mathrm{p}<0.001), 10 \mu \mathrm{g} / \mathrm{L}(\mathrm{p}<0.001)$, and $100 \mu \mathrm{g} / \mathrm{L}(\mathrm{p}=0.005)$. Despite this, there was no decreasing trend in the number of cells showing antigravitactic behaviour with increasing TBT concentrations, as the number of cells swimming up remained relatively unchanged throughout this 100-fold concentration range, and in fact showed a mild increase at the highest concentration, up from $30.9 \% \pm 2.6$ to $37.1 \% \pm 2.1$. To apply these results to the database of an early warning system for testing water quality, further studies are needed to determine the LOEC or NOEC, and whether higher concentrations of TBT will have the same effect on antigravitactic behaviour or whether this response plateaus at the concentrations studied.

\subsection{2.b. Velocity and TBT exposure}

To assess the sensitivity of the automated image analysis system's cell velocity parameter and whether it is comparable to visual observations of motility under the microscope, results of both tests should be compared. Results produced by either the ECOTOX system or by visual observation were not significantly different from reference. (Figure 3.10), therefore it cannot be said whether one is more sensitive than the other. The lack of significant results for this parameter suggests that swimming velocity as measured by automated image analysis cannot be used to determine the presence of TBT in an early warning system.

\subsection{2.c. Cell shape and TBT exposure}

Results of cell shape using the ECOTOX system yielded no significant results compared to reference (Figure 3.12), when previous research using visual observation had found effects on E. gracilis cell shape after $6 \mathrm{~h}$ exposure to $10 \mu \mathrm{g} / \mathrm{L}$ TBT (Pearce, 2009). This could suggest that the automated system is less sensitive at detecting morphological changes in cell shape than visual observation. Similarly, research by Ohta 
and Suzuki (2007) has found that E. gracilis Z strain's cell shape changes from elongated to cyst-form instantly (within 2 minutes) after addition of $50 \mu \mathrm{M}(298 \mathrm{mg} / \mathrm{L}) \mathrm{TBT}$. This is a much higher concentration than used for these experiments and higher than concentrations which would be found in the environment or in drinking water; therefore, significant instantaneous responses to TBT are unlikely to be found using either visual observations or image analysis. However, because no changes were detected with the automated system's cell shape parameter after TBT exposure at several concentrations when change had been detected previously, this parameter may not be appropriate for detecting the presence of TBT in an early warning system. Before this can be determined conclusively, further tests with the image analysis system are needed between $2 \mathrm{~h}$ and $24 \mathrm{~h}$ TBT incubation which were not included in this test to determine if there is an effect in cell shape as suggested by C. Pearce (2009) and cell morphology recovery after TBT exposure as suggested by Ohta and Suzuki (2007).

\subsection{2.d. $R$-value and TBT exposure}

Like velocity and compactness, results did not differ significantly from reference after analysis of randomness of swimming (Figure 3.13). As stated before, when the number of cells swimming upwards approaches $100 \%$ the r-value would too approach 1 , representing a non-random pattern of swimming. It was therefore expected that there would be a difference seen in r-values measured since there was a significant difference from reference in percent number of cells swimming up after 24h. It was also discussed in section 3.3.2.a that the r-value would be a more sensitive parameter than percent cells swimming up (Tahedl \& Häder, 1999; 2001). Again, this was not seen in this case. The rvalues found in this experiment ranged from 0.18 to 0.27 among both reference conditions and TBT treatment conditions, values which are closer to a random pattern of swimming (0) than non-random (1). The lower rage of r-values, interestingly, was found for reference conditions. There tended to be a high degree of variance for these data as well. These results were unexpected when considering what was found by Tahedl and Häder $(1999 ; 2001)$, and further tests using this parameter would necessary to determine 
whether its sensitivity makes the r-value appropriate for inclusion in an automated early warning system.

\subsubsection{Euglena gracilis automated parameters after copper exposure}

After $1 \mathrm{~h}$ and $2 \mathrm{~h}$ incubation in copper concentrations of $0.4,1.2,2$, and $20 \mu \mathrm{M}$, the behaviour of Euglena gracilis was analyzed using the ECOTOX system. The results are summarized in Figures 3.14 through 3.17.

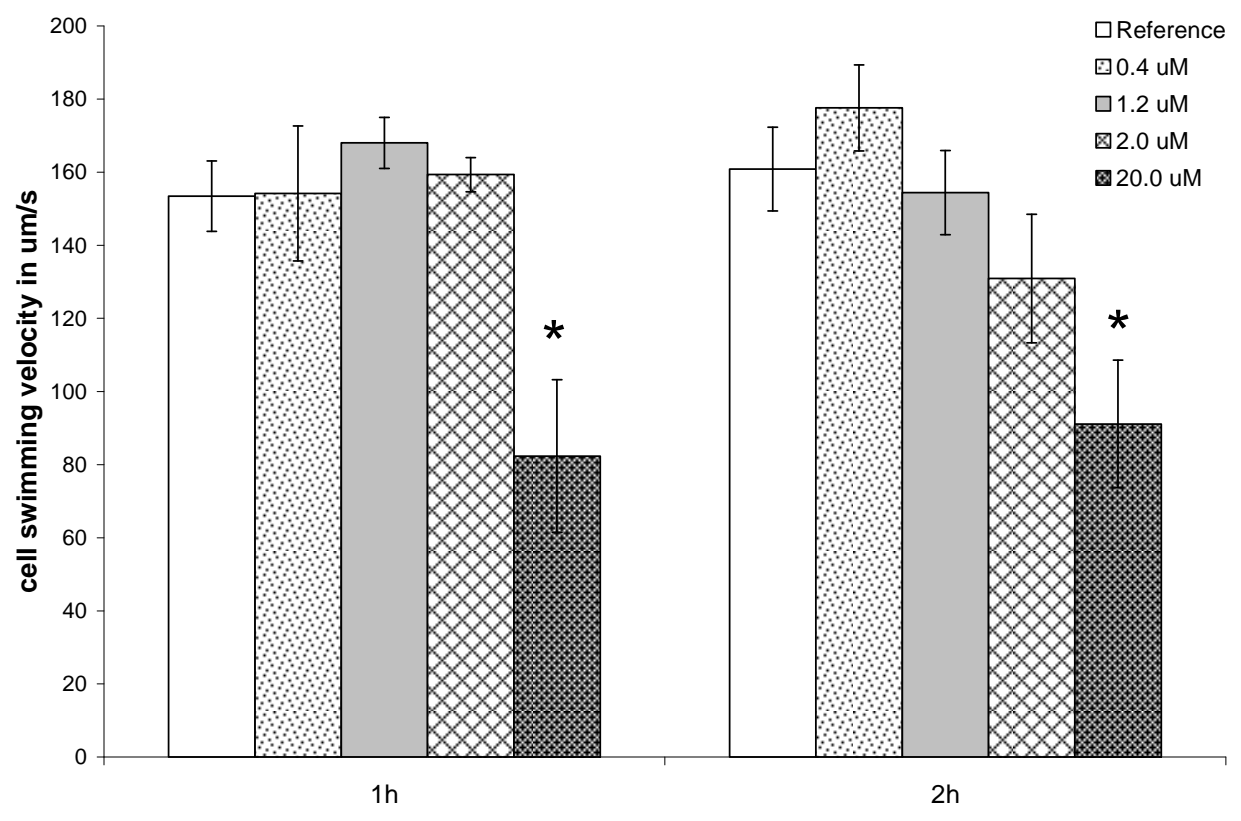

Figure 3.14. Euglena gracilis cell velocity after incubation in copper in 0.4, 1.2, 2, and $20 \mu \mathrm{M}$. Asterisks indicate results significantly different from reference. Bars indicate standard deviation. 


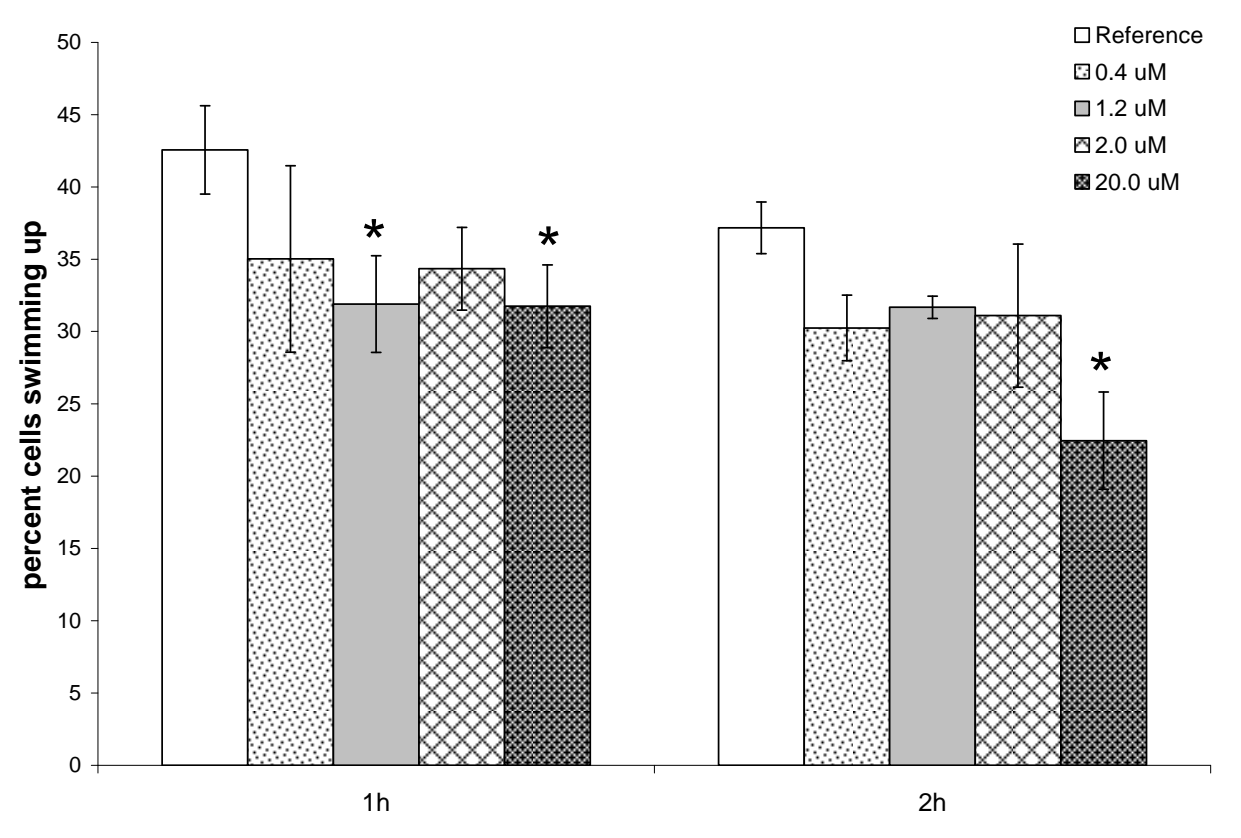

Figure 3.15. Euglena gracilis gravitactic orientation after incubation in copper in 0.4 , $1.2,2$, and $20 \mu \mathrm{M}$. Asterisks indicate results significantly different from reference. Bars indicate standard deviation.

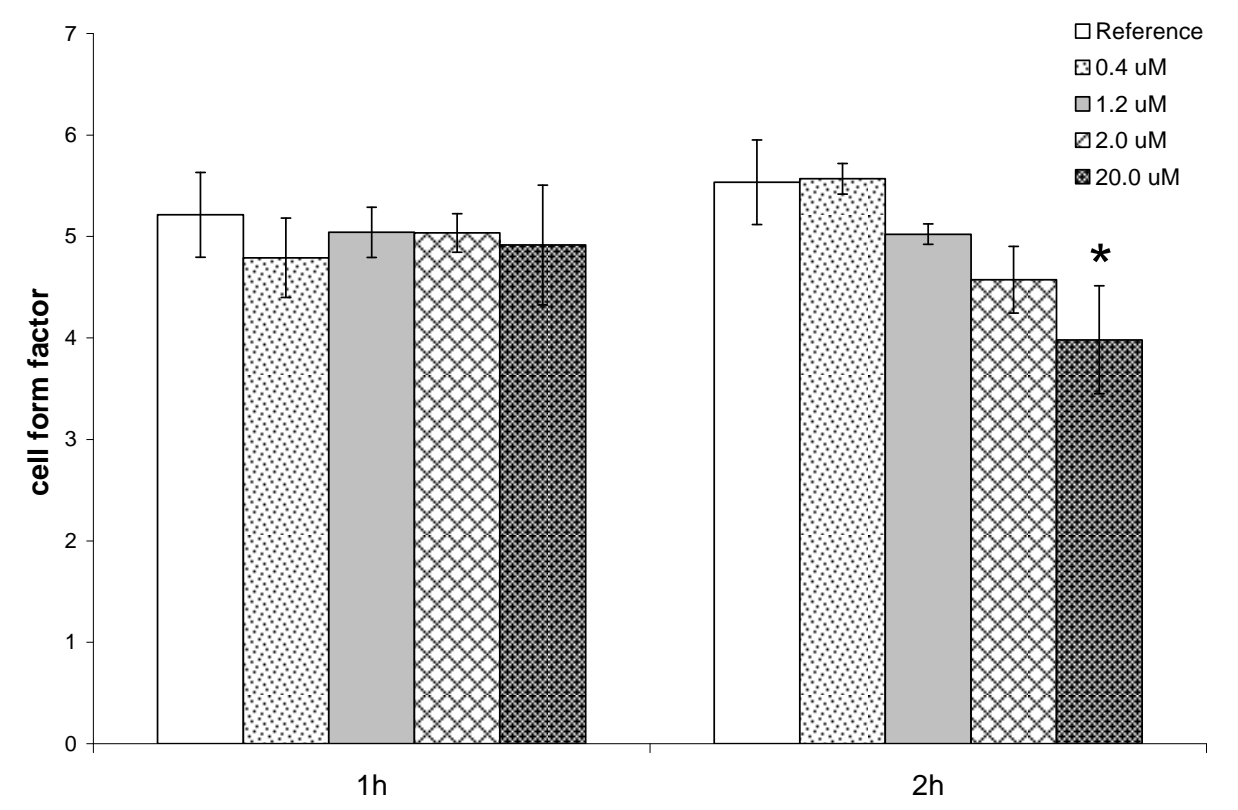

Figure 3.16. Euglena gracilis cell shape (decreasing numbers represent change towards cyst-form) after incubation in copper in 0.4, 1.2, 2, and $20 \mu \mathrm{M}$. Asterisks indicate results significantly different from reference. Bars indicate standard deviation. 


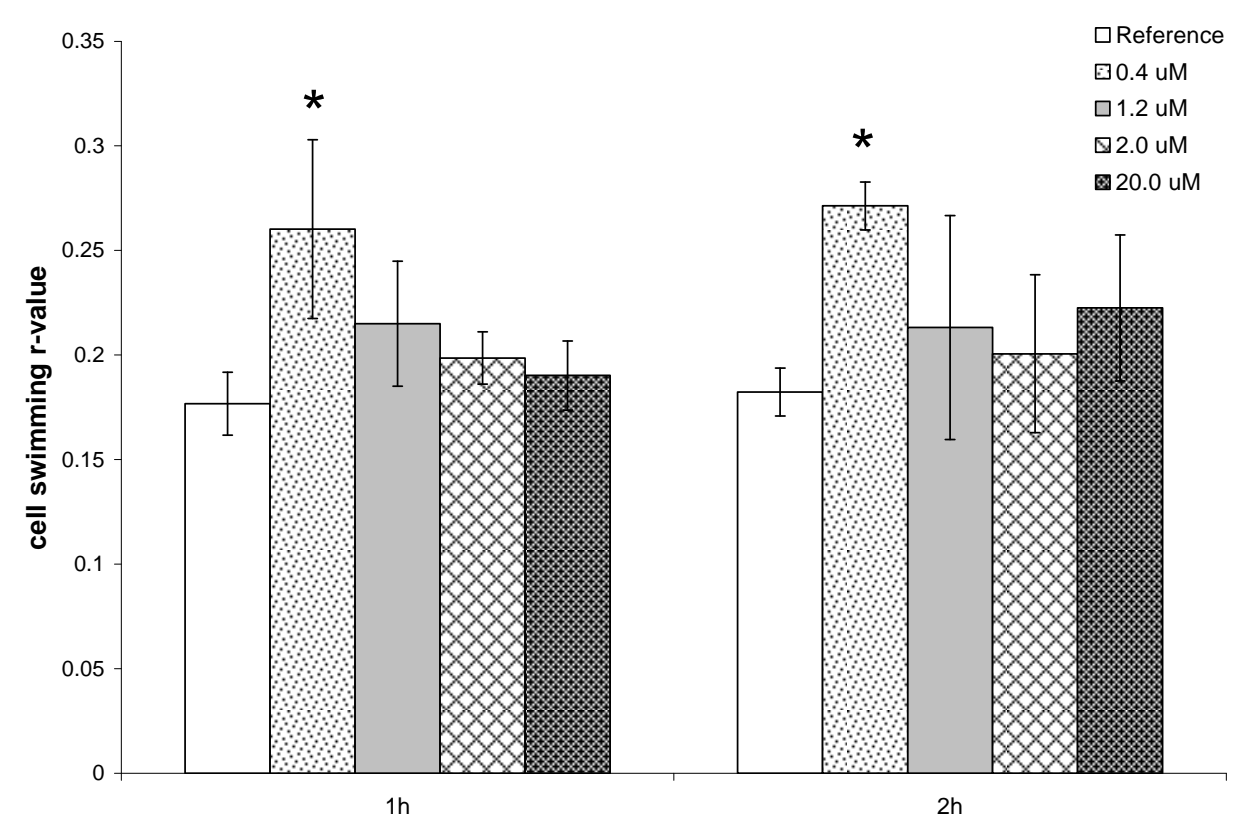

Figure 3.17. Euglena gracilis randomness of swimming (decreasing numbers represent more random swimming) after incubation in copper in 0.4, 1.2, 2, and 20 $\mu \mathrm{M}$. Asterisks indicate results significantly different from reference. Bars indicate standard deviation.

\subsection{3.a. Velocity and copper exposure}

When cell velocity was examined, it was found that it was significantly reduced from reference only at the highest concentration, $20 \mu \mathrm{M}$ (Figure 3.14). This sensitivity threshold is much higher than what was found using visual observations of cell motility, which was significant at $1.2 \mu \mathrm{M}$ at $21^{\circ} \mathrm{C}$ and $0.8 \mu \mathrm{M}$ at $26^{\circ} \mathrm{C}$. Measures of motility are related to measures of average swimming velocity in that as the number of motile cells decrease, so does the average value for cell swimming velocity. Therefore, because a significant difference was found in experiments of visual observation of cell motility previously at $0.8-1.2 \mu \mathrm{M}$, it was expected that a significant difference from reference in cell swimming velocity would be seen with similar concentrations, possibly in the $0.4-$ $2.0 \mu \mathrm{M}$ range. However, this was not the case. Visual observations looking at cells exposed to $20.0 \mu \mathrm{M}$ of copper for $1 \mathrm{~h}$ showed an almost complete reduction in motility, whereas cells incubated in the same concentration for the same length of time analyzed 
using the automated image analysis produced an average swimming velocity of 82.3 $\mu \mathrm{m} / \mathrm{s}$, only a $46 \%$ reduction from reference conditions, where average swimming velocity was $153.4 \mu \mathrm{m} / \mathrm{s}$.

One reason why this may be occurring is that nonmotile cells pumped into the cuvette are still recorded as swimming, since the cuvette is placed in the vertical position and cells which are not swimming are in fact falling due to gravity. However, from observing cells on the software screen, the speed of falling is still much slower than the speed of swimming and differences in speed should be detectable. One other possible explanation is that the nonmotile cells are reaching the cuvette in fewer numbers than the motile cells. It is possible that within the mixing chamber, nonmotile cells have time to settle to the bottom due to slow flow and not be pumped out into the cuvette. One observation that supports this idea is the fact that one can see a decreasing overall number of cells at increasing concentrations of copper on the software display of the interior of the cuvette on the computer screen. Another possibility is that nonmotile cells are adhering to "sticky" surfaces that perhaps exist inside the vinyl tubing, as there is some indication of cell accumulation along these tubes. Motile cells have also been observed inside the cuvette to stick to the surfaces and then unstuck after some amount of movement, which could mean that nonmotile cells remain stuck to surfaces because they do not have the movement necessary to release themselves. Further investigation is necessary to look at potential problems with E. gracilis cell swimming velocity measured by the ECOTOX system and to determine the reliability of this parameter and its suitability for an early warning system.

\subsection{3.b. Gravitactic behaviour and copper exposure}

When gravitactic behaviour of E. gracilis under copper stress was examined, significant differences from reference were found for exposure to concentrations of 20.0 $\mu \mathrm{M}$ after $1 \mathrm{~h}$ and $2 \mathrm{~h}$ incubation (Figure 3.15). After $1 \mathrm{~h}$ there was also a difference in the 1.2 $\mu \mathrm{M}$ treatment; however, this difference did not repeat for $1.6 \mu \mathrm{M}$ for the same incubation time, suggesting that this parameter is not consistent. This may also be related 
to issues of mixing motile and nonmotile cells mentioned previously which may have been affecting measured cell swimming velocity. Observation of the software screen during tests indicates that the inhibition in cells swimming upwards does not come from the direction of swimming itself but from the number of nonmotile cells on screen falling downwards in the cuvette due to gravity, which is not expected since the system should be able to detect cells which are nonmotile. The observed relative inconsistency encountered above supports the idea that nonmotile are not entering the observation cuvette in the same proportion as they are found in solution. It is also important to note that differences in percent number of cells swimming upwards only differed consistently from reference at concentrations of $20.0 \mu \mathrm{M}$, where the image analysis system found that $31.8 \%$ of cells were moving upwards after $1 \mathrm{~h}$ incubation in $20.0 \mu \mathrm{M}$ copper, and $22.4 \%$ after $2 \mathrm{~h}$ incubation. Since copper affects cell motility, the number of cells swimming up should be affected by the number of nonmotile cells, as the latter should not be able to move upwards. However, in contrast with observed cell motility under the microscope where cells were exposed to the same copper concentration, these numbers are conflicting: only $0.8 \%$ cells were motile after $1 \mathrm{~h}$ incubation, and only $1.5 \%$ after $2 \mathrm{~h}$. It is not possible that $31.8 \%$ of cells are moving upwards when only $0.8 \%$ are motile. This observation also supports the idea that by some mechanism the nonmotile cells in solution are not being pumped through all the way to the cuvette where they can be observed by image analysis. While differences in this parameter were ultimately found at concentrations of $20.0 \mu \mathrm{M}$, it is again much less sensitive than visual observations of motility. This parameter should only be included for copper detection in an early warning system after mixing and cell pumping issues have been investigated and resolved.

\subsection{3.c. Cell shape and copper exposure}

When E. gracilis cell shape was examined using the ECOTOX image analysis system, results differed from reference at $20.0 \mu \mathrm{M}$ after $2 \mathrm{~h}$ incubation (Figure 3.16). In contrast, through visual observation of cell shape, differences could be seen at $20.0 \mu \mathrm{M}$ copper after $1 \mathrm{~h}$ only, and after $2 \mathrm{~h}$ differences were noted at concentrations of $2 \mu \mathrm{M}$ and 
above, making this parameter more sensitive under microscope observation than when analyzed with automated image analysis using ECOTOX. This parameter may again be related to issues of nonmotile cells not reaching the observation chamber. Under visual observation of cells under copper stress, it can be seen that the majority of nonmotile cells are cyst-form, while all cyst-form cells should be nonmotile (Tahedl \& Häder, 1999). A difference in morphology can be seen in motile cells only as they change from elongated to oval. If there is indeed an issue of mixing of nonmotile cells in the solution, then this parameter would certainly be affected as well.

Previously Tahedl and Häder (2001) had found compactness to be the most sensitive parameter for assessing presence of copper using ECOTOX, where the $\mathrm{EC}_{50}$ found was $4.0 \mathrm{mg} / \mathrm{L}$ after 30 minutes incubation. However, in another study by Tahedl and Häder (1999), the $\mathrm{EC}_{50}$ value for copper was found to be double that, $8.0 \mathrm{mg} / \mathrm{L}$, and the most sensitive parameter was found to be r-value. The only difference between these was the form of copper used: the 1999 study used copper as $\mathrm{CuSO}_{4}$, whereas the 2001 study used $\mathrm{CuCl}_{2}$. It is not clear whether there had been any changes in the software in that time period. While cell shape may still be a good parameter for indicating copper stress, the newest study looking at E. gracilis behaviour under copper stress using the ECOTOX system has found that after 2 minutes incubation the most sensitive parameter was motility, followed very closely by r-value, with $\mathrm{EC}_{50}$ values of $19.09 \mathrm{mg} / \mathrm{L}$ and 20.4 $\mathrm{mg} / \mathrm{L}$ respectively (corresponding to 300.4 and $312.4 \mu \mathrm{M}$ ). The effects on E. gracilis cell shape under copper stress were, however, not investigated (Ahmed \& Häder, 2010). Values for the Ahmed and Häder study (2010) are higher than the previously described Tahedl and Häder study (1999) due to the shorter incubation time, but all $\mathrm{EC}_{50}$ values in these are considerably higher than the concentrations at which a significant difference in cell shape was found with this study, and also higher than $\mathrm{EC}_{50}$ values found for visual observation of E. gracilis motility. Nevertheless, it is shown that this parameter does show some sensitivity, which is comparable to cell swimming velocity and percent cells swimming up as measured by the ECOTOX system. Further studies must investigate the sensitivity of this parameter to other contaminants as well. 


\subsection{3.d. $R$-value and copper exposure}

When r-value was examined, a significant difference from reference was found at the lowest concentration, $0.4 \mu \mathrm{M}$, after $1 \mathrm{~h}$ and $2 \mathrm{~h}$ incubation $(\mathrm{p}=0.024$ and 0.023 respectively). Unlike the other parameters examined, r-value actually increases at this concentration (Figure 3.17). The r-value was found to be very close to or the most sensitive parameter when looking at E. gracilis behaviour under copper stress using ECOTOX in two separate studies (Tahedl \& Häder, 1999; Ahmed \& Häder, 2010). However, these studies had found inhibition of r-value towards a more random pattern of swimming, whereas this experiment yielded an increase in r-value. It is possible that this is a hormetic effect; however, at higher concentrations observed there was still no inhibition detected and r-values were very similar to reference conditions. This makes $r$ value an unreliable parameter when looking at copper toxicity at the concentration range examined.

\subsection{Suitability of the automated image analysis system ECOTOX}

To determine whether the ECOTOX system is a suitable automated behavioural analysis system for incorporation into an early warning system for drinking water quality, each behavioural parameter measured with this image analysis system was assessed individually to determine whether it is sensitive and reliable indicators of stress. The overall usability of the ECOTOX equipment itself is also discussed.

\subsubsection{Behavioural parameter: cell swimming velocity}

Cell swimming velocity detected the presence of copper, but not the presence of atrazine or TBT. Results from the visual observation of motility did not show inhibition of this behaviour at the concentrations of atrazine and TBT tested (Figures 3.6 and 3.10), and it was unknown whether the automated detection of velocity was more or less sensitive than the visual observation of motility. Since the automated system did not 
show any differences in velocity for these contaminants, it cannot be determined whether the automated system is more or less sensitive than the visual observation for detection of E. gracilis changes after exposure to these organic contaminants.

Differences were noted between the visual observation of E. gracilis motility in comparison with the automated measure of swimming velocity after incubation in copper. While clear responses could be seen under the microscope and an $\mathrm{EC}_{50}$ cold be obtained between 0.4 and $2 \mu \mathrm{M}$, the automated system only detected changes in swimming velocity at $20 \mu \mathrm{M}$. This indicates that the automated measure of velocity is not as sensitive as the visual observation, which, at $20 \mu \mathrm{M}$, demonstrated almost complete inhibition of motility. In fact even when the same sample was observed under the microscope and on the ECOTOX software screen simultaneously, there were distinct differences in the proportion of motile cells. As discussed in section 3.3.3, nonmotile cells in the ECOTOX system may not be reaching the observation cuvette either because they are settling in the mixing chamber or because they are sticking to tubing surfaces within the system.

A further problem with velocity exists in that in the data produced there are values that do not fit the E. gracilis natural swimming velocity range, which according to Checcucchi (1976b) ranges between 100 and $150 \mu \mathrm{m} / \mathrm{s}$ under normal conditions. These values did not appear so frequently as to change the average swimming velocity by a great extent, but nonetheless average velocities of up to $2400 \mu \mathrm{m} / \mathrm{s}$ were recorded with ECOTOX. For the purpose of this study all values above 2 standard deviations were removed, and this included most values above $300 \mu \mathrm{m} / \mathrm{s}$. Before this system is implemented in water quality testing facilities, it is necessary to investigate why it is producing these values. The possibility of culture contamination was eliminated, and even under the possibility of contamination it is unlikely that a few organisms would change the velocity to the extent found, since the velocity parameter is calculated as an average swimming velocity of many cells. Another possibility considered is that the sensitivity of the system is great enough to detect tremors such as are caused by closing doors and drawers. It also cannot be discounted that construction work in adjacent laboratories were causing vibrations that the system was detecting. These abnormally 
high speeds must continue to be investigated before it can be deemed suitable for implementation.

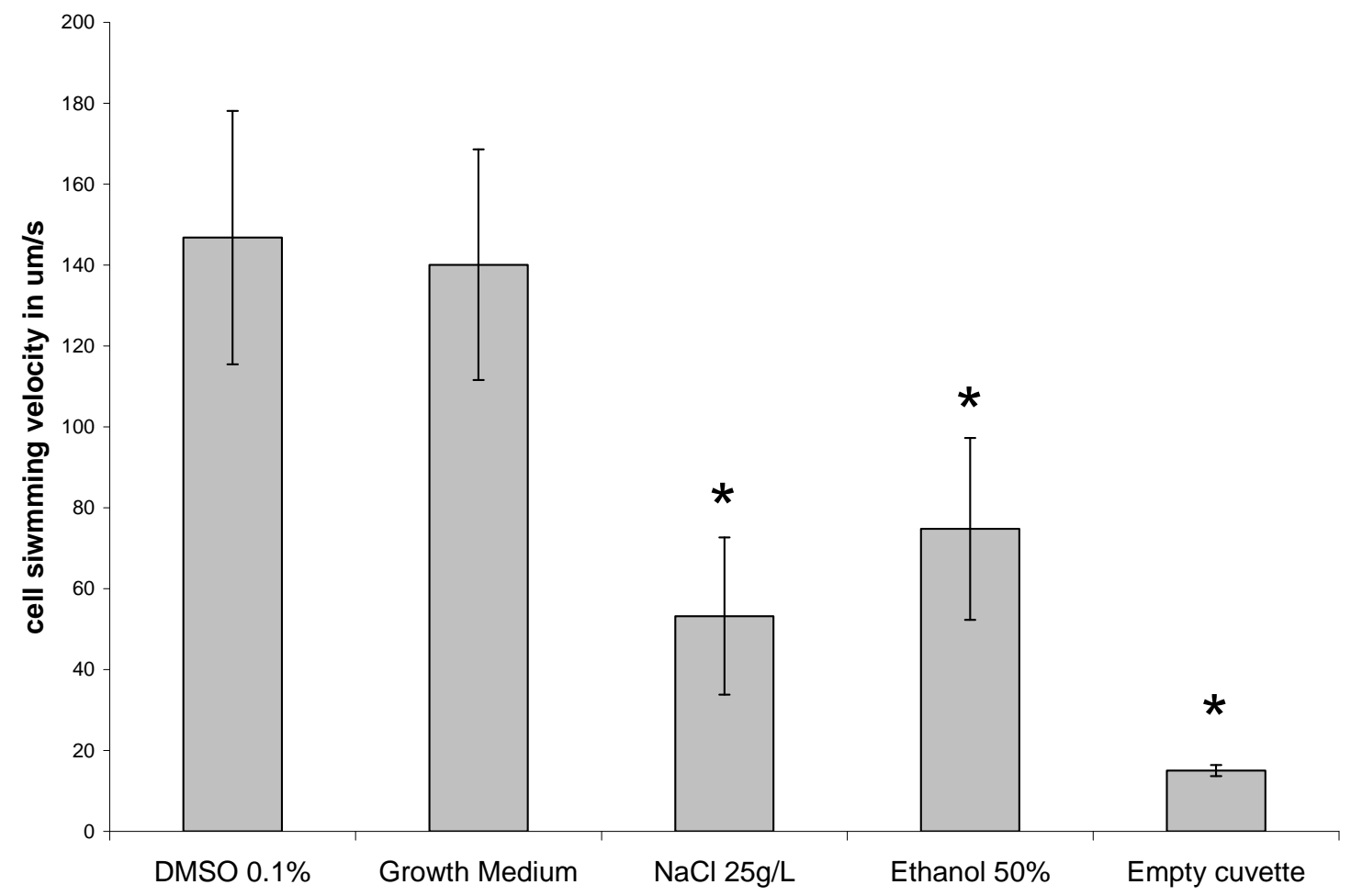

Figure 3.18. Euglena gracilis swimming velocity measured in $\mu \mathrm{m} / \mathrm{s}$ after 5 minutes exposure to several controls and in comparison to readings from an empty cuvette. Growth medium and $0.1 \%$ DMSO are considered normal reference conditions. Visual observation indicated that none of the cells in $25 \mathrm{~g} / \mathrm{L} \mathrm{NaCl}$ or $50 \%$ ethanol were motile. Asterisks indicate results significantly different from reference.

The ECOTOX system should be able to measure the percentage of cells that are motile versus nonmotile as well, as several papers using ECOTOX as a measure of $E$. gracilis behaviour have used the motility parameter (Tahedl \& Häder, 1999; Tahedl \& Häder, 2001; Ahmed \& Häder, 2010). The software can be used to set a minimum speed at which cells are considered motile. In this study this number was set to $20 \mu \mathrm{m} / \mathrm{s}$. However, motility was not used as a comparative parameter because for all trials the automated system was recording this value as $100 \%$ motile cells, and this remained unchanged for all trials and contaminants tested. Figure 3.18 shows swimming velocity results for controls which were used to measure each of the parameters for proper 
functioning. $25 \mathrm{~g} / \mathrm{L} \mathrm{NaCl}$ and $50 \%$ ethanol were chosen as controls for causing a complete reduction in motility. From the graph it can be seen that swimming speed is partially but not completely reduced. After visual observation of the software screen it can be seen that cells are indeed drifting despite being nonmotile. Under the $25 \mathrm{~g} / \mathrm{L} \mathrm{NaCl}$ treatment all cells are moving in an upwards direction as there are density differences due to the salt concentration and all cells are floating up, whereas under the $50 \%$ ethanol treatment all cells are nonmotile but appear to be sinking due to gravity, as the observation cuvette is placed in the vertical position and nonmotile cells do not remain buoyant in the water column. The same issue could be observed with cells at $20 \mu \mathrm{M}$ copper, where some nonmotile cells appeared on the software display, but rather than nonmotile they were seen as sinking downwards due to gravity.

One possible way to reduce this error is to have a cuvette placed horizontally. Because it is necessary to have a vertically placed cuvette to measure gravitactic behaviour, one possibility is to have two cuvettes, one vertical and one horizontal, being measured in parallel to measure motility/velocity and gravitactic behaviour separately. Figure 3.18 also indicates that some movement is being detected within an empty cuvette. Visual observation indicates that despite brightness and contrast adjustments, and despite inputting minimum size adjustments in the software, that the software is still picking up artifacts which are not there (as indicated by the green line highlighting the object being measured). This does not seem to have a strong effect on results when cells are actually present, but it is certainly an issue which can be improved before this system is implemented.

\subsubsection{Behavioural parameter: gravitactic orientation}

Gravitactic orientation, measured in percent cells swimming up, was the most sensitive parameter for changes occurring after $24 \mathrm{~h}$ incubation in the organic contaminants tested, atrazine and TBT, and in fact the only sensitive parameter after exposure to these two contaminants. This parameter was not found to be the most sensitive when looking at a variety of contaminants using the ECOTOX system (Tahedl 
\& Häder, 1999; 2001), which makes these results unexpected. Results for this parameter are the most positive for the ECOTOX system, as it was able to detect behavioural changes that visual observation under the microscope could not.

The gravitactic orientation parameter was also able to detect changes at $20 \mu \mathrm{M}$ of copper after $1 \mathrm{~h}$ and $2 \mathrm{~h}$ incubation. It detected changes at $1.2 \mu \mathrm{M}$ as well but not at $2 \mu \mathrm{M}$, indicating some inconsistency at low concentrations. At the higher concentration, $20 \mu \mathrm{M}$, visual observation of the software display determined that the number of cells swimming up in this case was determined in general by a higher number of nonmotile cells in the observation chamber. The same conditions were found when the system was measuring $50 \%$ ethanol exposure (Figure 3.19). If this number of nonmotile cells corresponded to the number of nonmotile cells in the mixture as observed under the microscope, this would strengthen the sensitivity and reliability of this parameter.

A few issues still remain with measuring percent cells swimming up. As can be seen in Figure 3.19, after $25 \mathrm{~g} / \mathrm{L} \mathrm{NaCl}$ exposure, the number of cells recorded as swimming up increases rather than decreases. As discussed in section 3.4.1, this occurs due to the density gradient between the cells and the salt solution. This is not a concern when applying the system for drinking water quality testing. As with velocity, when looking at an empty cuvette the system seems to pick up movement that is not there; however this does not seem to be affecting the other measurements, and issues with measuring this parameter appear to be related mostly with the vertical position of the cuvette. With improvements in the cell mixing process inside the ECOTOX system, gravitactic behaviour has the potential to be the most sensitive parameter for detecting presence of contaminants, and also a discriminating parameter when changes are seen after $1 \mathrm{~h}$ or $2 \mathrm{~h}$ incubation (copper) or $24 \mathrm{~h}$ incubation (atrazine and TBT). This is in agreement with previous research by Häder, who developed the ECOTOX system with gravitaxis as the most important parameter in mind (Lebert \& Häder, 1999; Richter et al., 2001; Häder, 2003). 


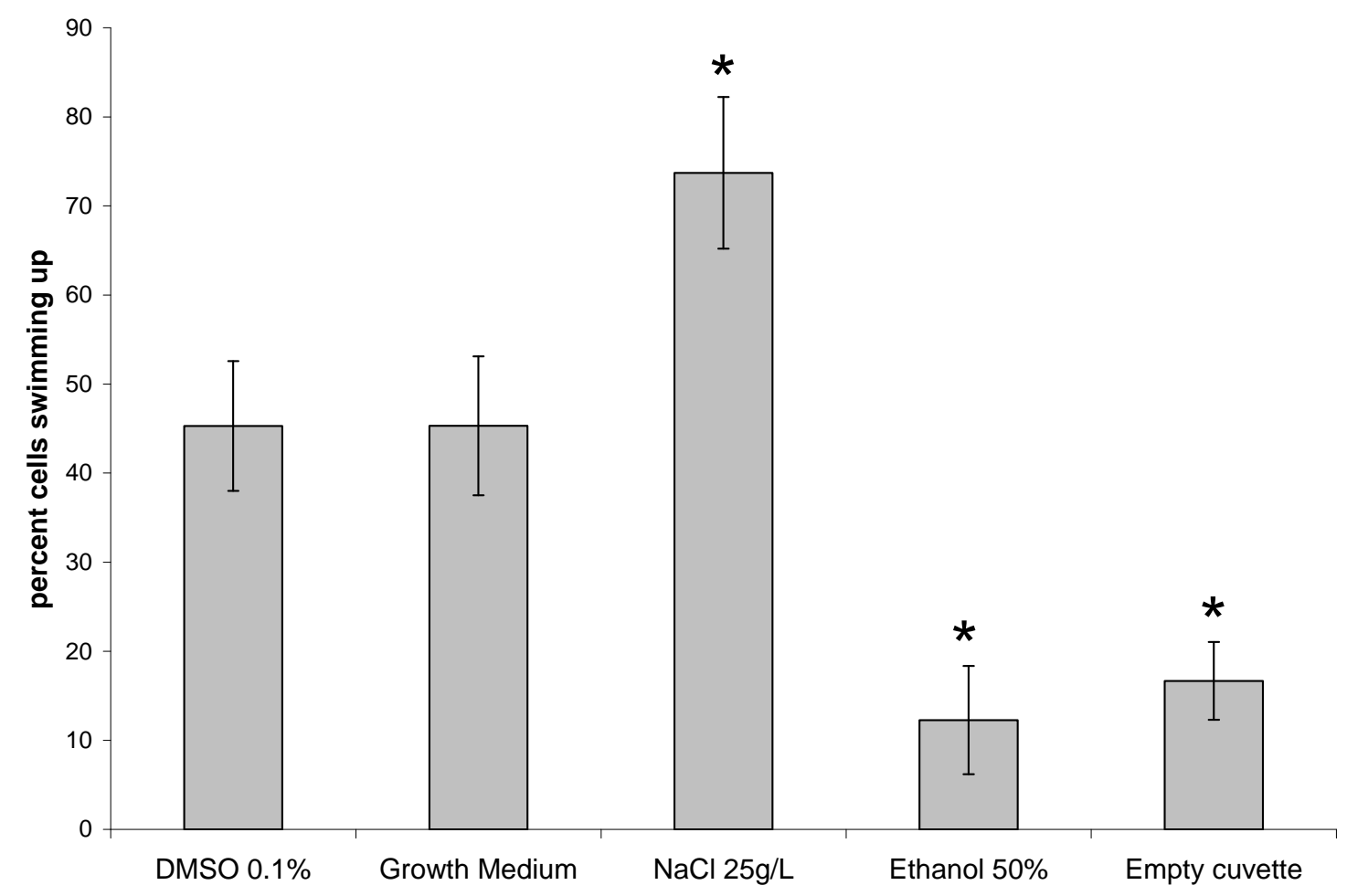

Figure 3.19. Euglena gracilis gravitactic behaviour (measured in percent cells swimming up) after 5 minutes exposure to several controls and in comparison to readings from an empty cuvette. Growth medium and 0.1\% DMSO are considered normal reference conditions. Asterisks indicate results significantly different from reference.

\subsubsection{Behavioural parameter: cell shape}

The cell shape parameter detected the presence of copper at $20 \mu \mathrm{M}$. Results from the automated system's observation did not show cells changing shape towards cyst-form at the concentrations of atrazine and TBT tested (Figures 3.8 and 3.12), despite the fact that differences had been found in previous research (Pearce, 2009). Comparison of copper results was used to determine whether the automated detection of cell form factor/shape was more or less sensitive than the visual observation of cell shape. Differences in visual observation under the microscope (Figure 3.5) became significant at $20 \mu \mathrm{M}$ after $1 \mathrm{~h}$ and $2 \mu \mathrm{M}$ after $2 \mathrm{~h}$, whereas the automated system (Figure 3.16) only 
detected changes at $20 \mu \mathrm{M}$ after $2 \mathrm{~h}$, making the visual observation slightly more sensitive than the automated system's.

This difference likely exists because of the difference in methodology used to assess cell shape. Whereas in the visual observation one assigns discrete values to different shape categories, the automated system calculates the cell's circumference to area ratio. In Figure 3.20 one can compare the automated system's cell form factor in reference conditions with form factor in $\mathrm{NaCl}$, where it was determined by visual observation of the software display that all cells were in cyst-form. The difference between the two is less than $50 \%$ inhibition. When comparing the effects of copper on cell shape by visual observation under the microscope, Figure 3.5 shows more than $50 \%$ inhibition between reference and $20 \mu \mathrm{M}$ after $1 \mathrm{~h}$, whereas Figure 3.16 (ECOTOX automated system) shows no inhibition at this concentration after $1 \mathrm{~h}$ incubation and less than $30 \%$ inhibition after $2 \mathrm{~h}$.

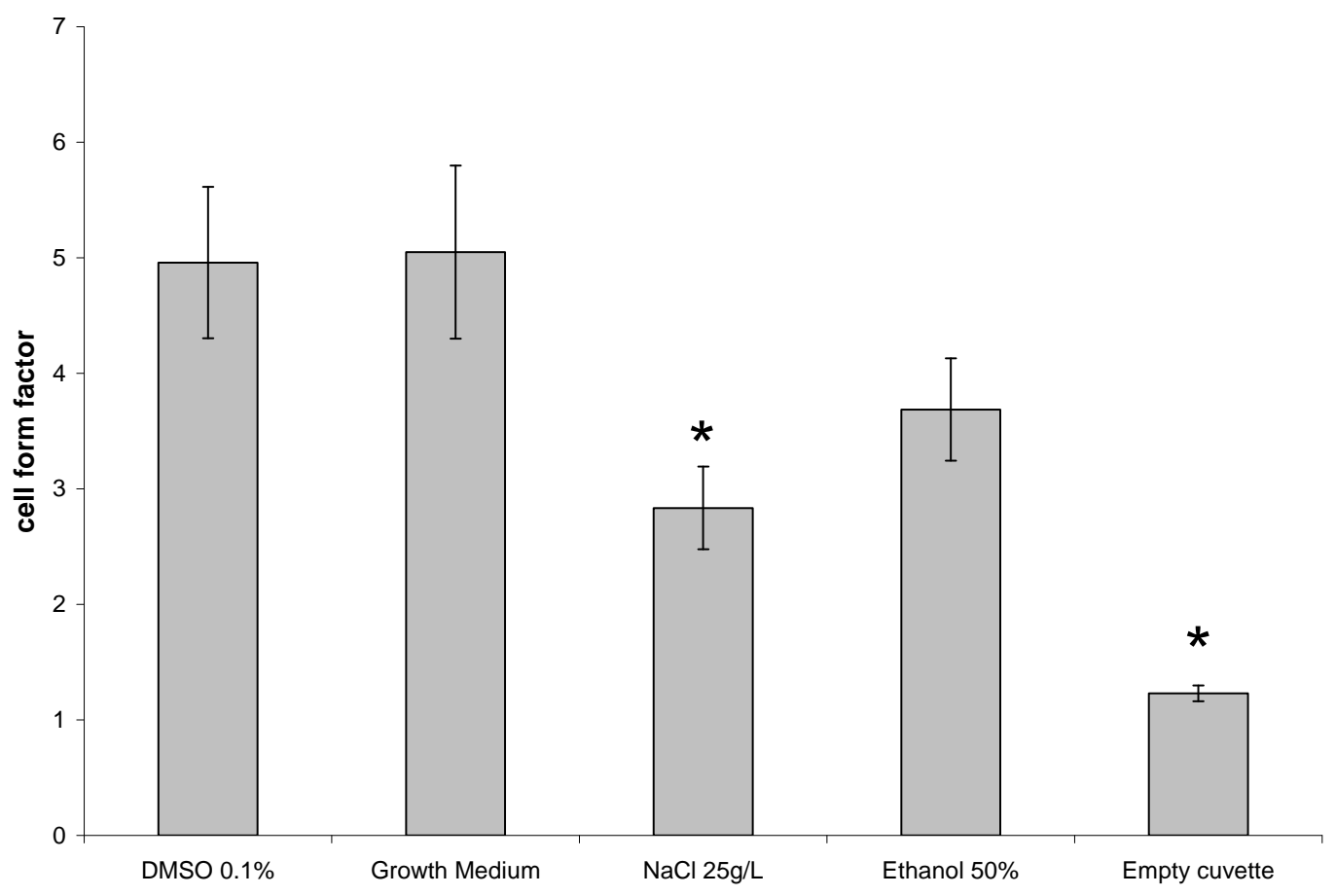

Figure 3.20. Euglena gracilis cell shape (measured in form factor) after 5 minutes exposure to several controls and in comparison to readings from an empty cuvette. Growth medium and $0.1 \%$ DMSO are considered normal reference conditions. Asterisks indicate results significantly different from reference. 
This parameter also seems to be related to motility, as cyst-form cells are nonmotile. However, motility remains a more sensitive parameter because all cyst-form cells are nonmotile but not all nonmotile cells are cyst-form. It is possible that this connection and the lower sensitivity may make this parameter redundant if an optimal method for measuring cell motility is implemented. The ECOTOX system itself produced results showing that cell velocity was quicker in responding to copper toxicity than cell form factor, so this redundancy may apply to the automated system as well.

\subsubsection{Behavioural parameter: randomness of swimming}

The randomness of swimming was found to be the most sensitive parameter for most contaminants tested in previous research by Tahedl and Häder (1999; 2001). This principle is based on the idea that under normal conditions, E. gracilis will show antigravitactic swimming orientation and most cells will swim upwards, whereas under stress conditions they tend to swim in random direction. However, this is not what was found for these experiments. For all trials, all cells including cells in reference conditions had very low r-value, closer to 0 than to 1 , meaning a more random pattern of swimming (Figures 3.9, 3.13, and 3.17). When testing for copper, the r-value increased at $0.4 \mu \mathrm{M}$, meaning the randomness decreased for that concentration, but for higher concentrations the randomness of swimming was again equivalent to reference conditions. This suggests either hormetic effects at $0.4 \mu \mathrm{M}$ or an inconsistency of the parameter itself. In either case this parameter is still not adequate for measuring E. gracilis behaviour under exposure to the contaminants tested. The fact that at $20 \mu \mathrm{M}$ the system detected changes in percent cells swimming up (Figure 3.15) but not in r-value (Figure 3.17) suggests that the latter is less sensitive.

Figure 3.21 shows a less random pattern of cell movement for $\mathrm{NaCl}$ and ethanol which is related to a decline in motility only. In both treatments all cells are nonmotile, in $\mathrm{NaCl}$ all cells are moving upwards due to the density gradient, and in ethanol all cells are moving downwards due to gravity. 


\subsubsection{ECOTOX hardware and software stability}

The purpose of experiments using the ECOTOX image analysis system is to investigate its suitability for an early warning system for drinking water quality. Automated systems are ideally implemented in this setting because they can produce recordings while being largely unsupervised, where no daily maintenance is necessary. Ideally an automated system would need supervision by a person no more than once a week, such as the Daphnia Toximeter by bbe moldaenke GmbH. For this reason it is important to assess whether this system is robust enough to dispense daily monitoring and upkeep.

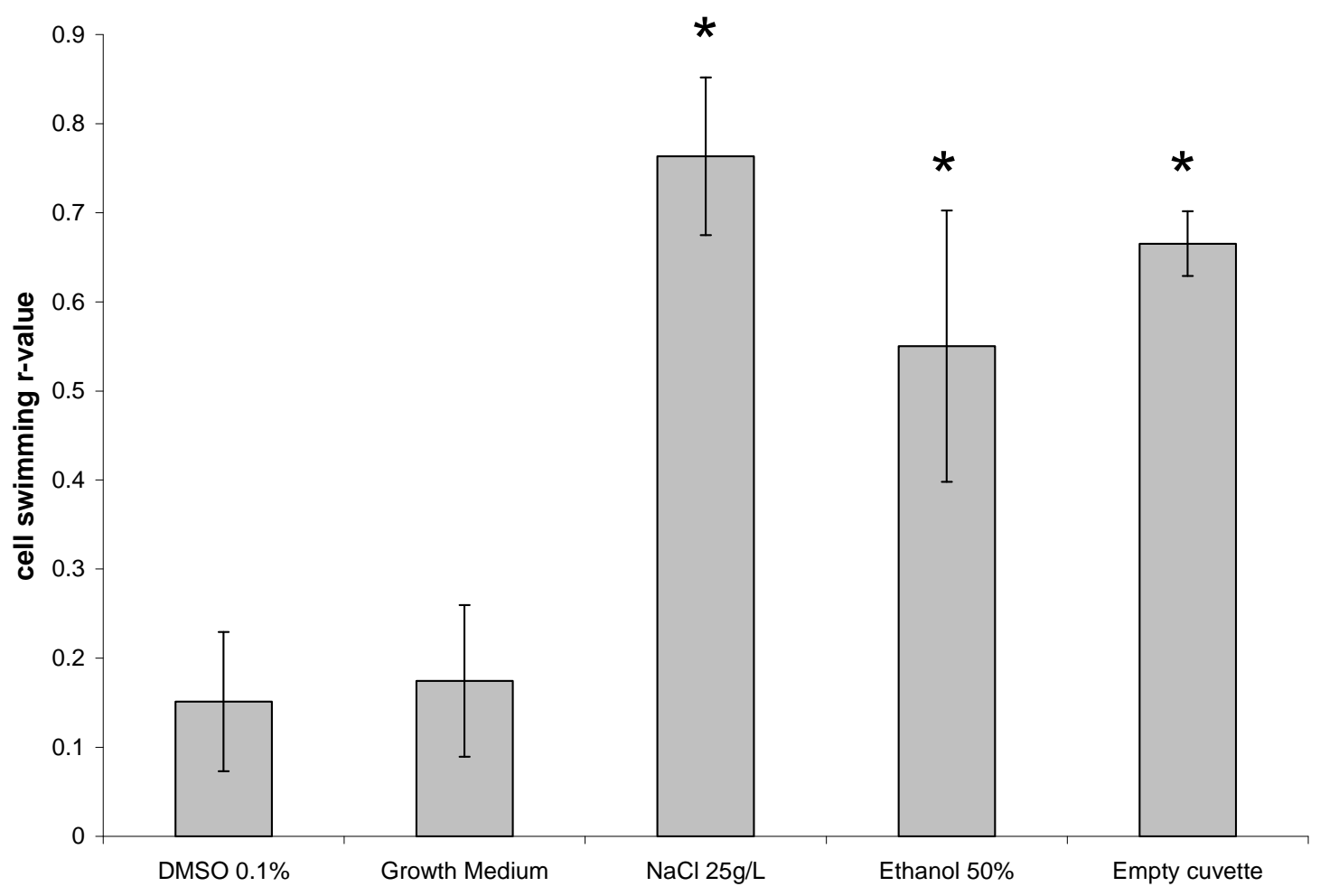

Figure 3.21. Euglena gracilis randomness of swimming (measured in r-value) after 5 minutes exposure to several controls and in comparison to readings from an empty cuvette. Growth medium and 0.1\% DMSO are considered normal reference conditions. Asterisks indicate results significantly different from reference. 


\subsection{5.a. Software and hardware maintenance}

The ECOTOX software was stable as long as instruments were connected properly and the E. gracilis culture density used for experiments were kept at $3.0 \times 10^{6}$ cells per $\mathrm{mL}$. Software error occurred when cell density was too high for tracking, and when the hardware was improperly connected.

A)

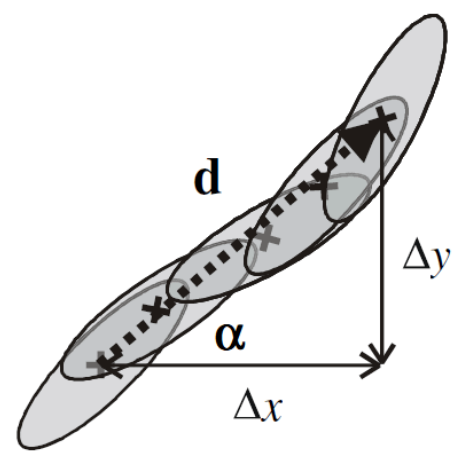

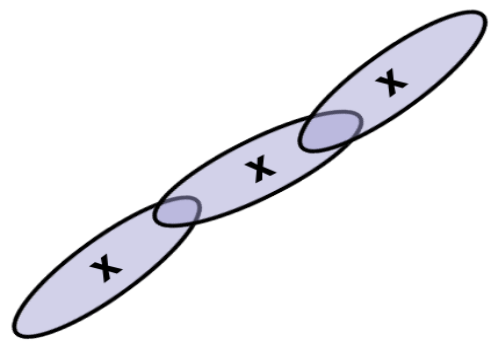

B)

Figure 3.22. The calculation of parameters on ECOTOX is based on a "track" (A), which is measured if the centre of gravity on one frame overlaps the area of the cell in the previous frame. In B) the centre of gravity of the $3^{\text {rd }}$ and $4^{\text {th }}$ frames do not overlap with the area of the previous image and is therefore not tracked.

The ECOTOX software can track up to 1500 objects per minute depending on the computer on which it runs (ECOTOX 5.0 manual). One "track" is understood to be a sequence of five frames, each taken at $40 \mathrm{~ms}$ apart, whereby a cell movement vector is determined by connecting the centre of gravity of the "object" or cell in one image to the centre of gravity of the cell in the subsequent image that falls within the area of the object in the previous image (Figure 3.22). This means that if the centre of gravity of the cell in the second frame does not fall within the area of the object in the first frame, then this track will be lost and not used by the system to calculate parameters (Tahedl \& Häder, $2000 ; 2001)$. This vector, along with the time taken, is then used to calculate all parameters given by the ECOTOX system

The ECOTOX hardware requires some upkeep. The silicone tubing erodes with time and cracks occur, causing leaks. The system includes a leak detection alarm, and on 
the occasion that it did sound off in the lab it was caused by large volumes of water spilled, whereas leaks with smaller volumes did not trigger the alarm. Therefore it is recommended that the silicone tubing be replaced every 3 or 4 months. The leaks were caused either by cracked tubing or due to the waist flask not being emptied in a timely fashion.

\subsection{5.b. Quality of ECOTOX manual and technical support}

The ECOTOX manual is fairly reliable, although on some instances the technical support contact, M. Lebert (University of Erlangen-Nuremberg), needed to be contacted. One of these instances was with regard to the recommended cleaning agent, Clorax (sodium chlorate). It was considered safe for the materials used in ECOTOX components

and recommended (M. Lebert, personal communication, October 2009), but was not used due to concerns of possible formation of organochlorinated compounds after atrazine exposure (K. Terry, personal communication, October 2009). Further contact needed to be made with regard to inconsistencies in the manual with the concentrations of serial dilutions, where it was determined that the proportions of contaminant to culture were determined to be different than those that were published in the manual and in previous research.

\subsection{5.c. Weekly upkeep for water quality testing}

A few things are important to note if the system is to run continuously in between weekly monitoring. There needs to be a sufficient amount of E. gracilis culture, and sample water available for testing throughout the week, and the waste needs to be consistently drained. The amount of culture and water used and waste produced varied depending on the pump settings, but in the context of 7 days unmonitored recording this cannot be contained within the ECOTOX system. All of these have to be externalized. The simplest way to do this is to create openings for the tubing to be pulled outside the enclosed ECOTOX system. To provide enough culture for one full week of testing, the 
system will ideally be connected to an E. gracilis chemostat providing continuous culture

growth, with controlled population density. Other automated monitoring systems already incorporate chemostats, such as the bbe Daphnia Toximeter, which contains one for algae used for feeding D. magna throughout the week. Maintenance and supervision is therefore limited to only weekly replacement of organisms (R. Engi, personal communication, May 2010). If using flask cultures, they would still need to be brought outside the enclosed box containing the system, as long periods of time without light exposure is likely to affect the organism. The cell density would also not be controlled in this case. Other changes will also improve the system; the waste tubing should be connected to an automatic draining tube so the waste container does not have to be replaced daily. Further, the sample water tubing should be connected to a constant source of water to be tested. Two options exist for this, one is to attach the tube to a continuous flow source and test instant behavioural changes which occur after 5 minutes incubation within the ECOTOX system. The second option is similar to what is in place with the bbe Daphnia Toximeter in the groundwater plant Hardhof, Zurich Water Supply, Switzerland (R. Engi, personal communication, May 2010) - organisms are tested daily for $24 \mathrm{~h}$ exposure in water, allowing organisms to respond to contaminants and/or concentrations that produce a slower response time, such as organic contaminants. Using E. gracilis and the ECOTOX system, a culture sample could be diluted in a 1:1 proportion with the water to be tested, and measurements could be carried out hourly where organisms were taken from that sample.

\subsection{5.d. Resilience and sensitivity of the ECOTOX system}

Equipment malfunction must also be considered, and plans must be in place to repair and/or replace parts as needed. During the testing months with this system, one major malfunction occurred with the motorized peristaltic pump used to draw the "sample water" which contained the contaminant. This made it impossible to use the system in Single Toxin mode, as it performs serial dilutions of contaminants. A parts replacement plan was not in place, and while after some effort the pump motor resumed 
working a few months later, it was not turning smoothly. The Single Toxin mode was therefore not utilized for experiments, as a pump malfunction would likely compromise the serial dilutions it is set to perform. The peristaltic pump for E. gracilis culture also seemed to not be turning smoothly; however, since it did turn continually, and the proportion of culture in the mixture was kept constant throughout experiments, this was thought to not affect the experiments negatively. If the system is to be implemented for water quality testing, replacement parts including peristaltic pump motors must be available at hand, and a trained technician must be available for equipment maintenance and assistance.

As discussed in section 3.4.1, it is possible that the system is sensitive to tremors. This must be further investigated, and, if indeed there is a high sensitivity, the laboratory space in which the system is kept must be adapted to reduce tremors and vibrations which may inadvertently affect the behaviour readings. The laboratory space must also be strongly temperature controlled due to the sensitivity of E. gracilis to temperature changes, as discussed in section 3.1.3.a.

\subsection{Suitability of the automated image analysis system DaphniaTox}

The DaphniaTox image analysis system was investigated for possible use in an early warning system for drinking water quality. No results were produced with this system, as it could not produce sufficient results due to software instability, which proved to be an issue serious enough to make the system unusable.

\subsubsection{DaphniaTox software}

The DaphniaTox software was installed in the same manner as the ECOTOX software; however, issues were present with this program from the first installation. Several problems occurred during a three month trial phase of the experiments. First, there were issues with file naming, where the time stamp on the file did not replace the 
previous time stamp but was instead added to the previous time stamp, creating longer and longer file names until they were no longer accepted by the operating system. This is a problem when the system should be able to run continuously, as when files are no longer accepted and saved, the system will effectively stop functioning. Another problem encountered was the run time of experiments. The software is set up to take continual measurements until the operator manually stops them through the software. However, the run time was inconsistent and behavioural measurements would inexplicably stop any time between 50 minutes and 80 minutes after the start time, rather than run continuously. Finally, the most serious problem proved to be software crashing during readings, which would happen approximately 9 out of 10 times the start command was selected, often during the first two readings.

To resolve these problems, software installation was attempted on several different computers with different operating systems such as Windows XP, XP Professional, and Vista, but these attempts were not successful. These issues were partly resolved by weekly reinstallation of drivers and software, as well as installation of new versions of the software as provided by M. Lebert (personal communication, November 2009). However, after a few months these measures ceased to repair the problems, and the system was no longer used.

\subsubsection{DaphniaTox parameters}

The DaphniaTox system measures both Daphnia magna swimming velocity and swimming orientation (percent cells swimming upwards and r-value), as well as phototactic behaviour. The system turns on a blue light above the test chambers, and measurements produce a separate file for these parameters under the regular red LED as well as under the blue phototactic LED. Preliminary observation of video recordings of this parameter showed that D. magna react immediately once the blue LED light is turned on, and in less than one second they begin swimming upwards. More biological processes are thought to be involved in phototactic movement than regular movement (Semsari \& Megateli, 2007), therefore this parameter has the potential to be more sensitive than 
looking at individual movement alone. This may be an advantage over other image analysis systems; however, software issues in general prevent this system from being used currently and further investigation is needed. Other image analysis systems do not automatically measure phototactic responses of D. magna. The bbe Daphnia Toximeter for example, a well-established D. magna behaviour image analysis system, measures many parameters including average swimming speed, swimming height, speed distribution, average distance between organisms, fractal dimension (number and tightness of turns), motility, distribution in chamber, and size (bbe Moldaenke, n.d.). However, it does not measure phototactic orientation of D. magna. This may put the DaphniaTox at a competitive advantage in the market for measuring an important parameter that has been shown to be sensitive (Michels et al., 1999; Kieu et al., 2001; Martins et al., 2007).

\subsection{Case study: implementation of an image analysis system in an early- warning system for drinking water in Zurich, Switzerland}

An interview was conducted with R. Engi on May 25, 2010, at the Hardhof groundwater plant in Zurich, Switzerland, where a biological early warning system is in place utilizing the bbe Daphnia Toximeter automated behavioural analysis system. The purpose of this interview was to provide a framework with which to base optimal implementation strategies for a biological early warning system in Ontario.

The Hardhof groundwater plant is locater within the Zurich city limits and provides $15 \%$ of the city's drinking water supply. The remaining source of drinking water comes mainly from the Limmat river, which flows into Lake Zurich. A biological early warning system is in place for the groundwater plant only. The groundwater is not treated but rather held in reservoirs for $24 \mathrm{~h}$ before it is sent into the water supply. Several physical parameters are monitored daily: temperature, $\mathrm{pH}$, redox potential, electric conductivity, and oxygen content. Chemical analyses for key substances such as chlorine are performed weekly. 
The bbe Daphnia Toximeter has been implemented at the Hardhof plant since 2006. A vaulted, temperature- and pressure-controlled lab holds the Daphnia Toximeter (Figure 3.23), while organisms are grown in a separate Zurich Water Supply laboratory. The Daphnia Toximeter (Figure 3.24) measures D. magna swimming parameters such as swimming velocity, swimming heights, turns and circling movements, grouping, and motility.

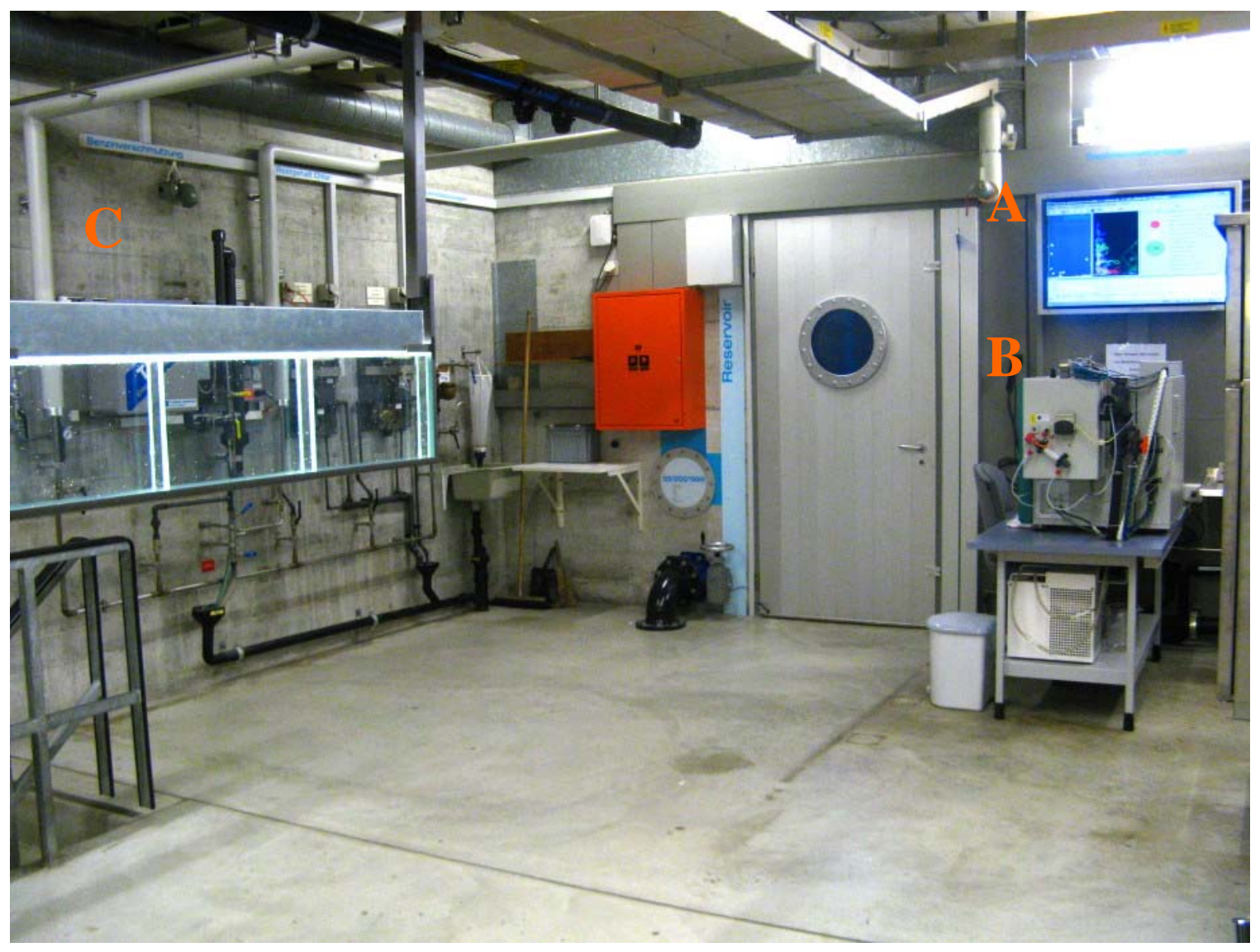

Figure 3.23. Laboratory at the Hardhof groundwater plant in Zurich, Switzerland, showing: A) the bbe Daphnia Toximeter display screen; B) the bbe Daphnia Toximeter hardware; and C) D. magna retirement aquaria.

At the Hardhof plant the Daphnia Toximeter is not used as a flow-through system but rather the organisms are allowed to incubate in the water tested for $24 \mathrm{~h}$, and readings are taken throughout this time to allow for investigation of substances which may take a 
longer time to affect D. magna. Four staff are responsible for all aspects of the Daphnia Toximeter maintenance, including culturing of organisms, replacement of organisms, and regular testing to ensure appropriate functioning of the system. Ten organisms are used per chamber, and four chambers are contained in the systems (Figure 3.24). Individuals are placed in the system at 3 days of age and replaced weekly at 10 days. The organisms are fed automatically from an algae fermenter included inside the system. An alarm is programmed to trigger when two or more parameters deviate from the norm, such as loss of motility and a decrease in velocity. According to R. Engi, in the 4 years that the system has been in place, the alarm has not been set off. The strategy in place for dealing with a potential alarm trigger is to shut off the plant and immediately stop supply to the Zurich drinking water system until chemical analyses can determine the safety of the water. This strategy can be implemented because the Hardhof plant only supplies 15\% of Zurich's drinking water and therefore the drinking water supply would not be drastically affected by shutting off this plant.

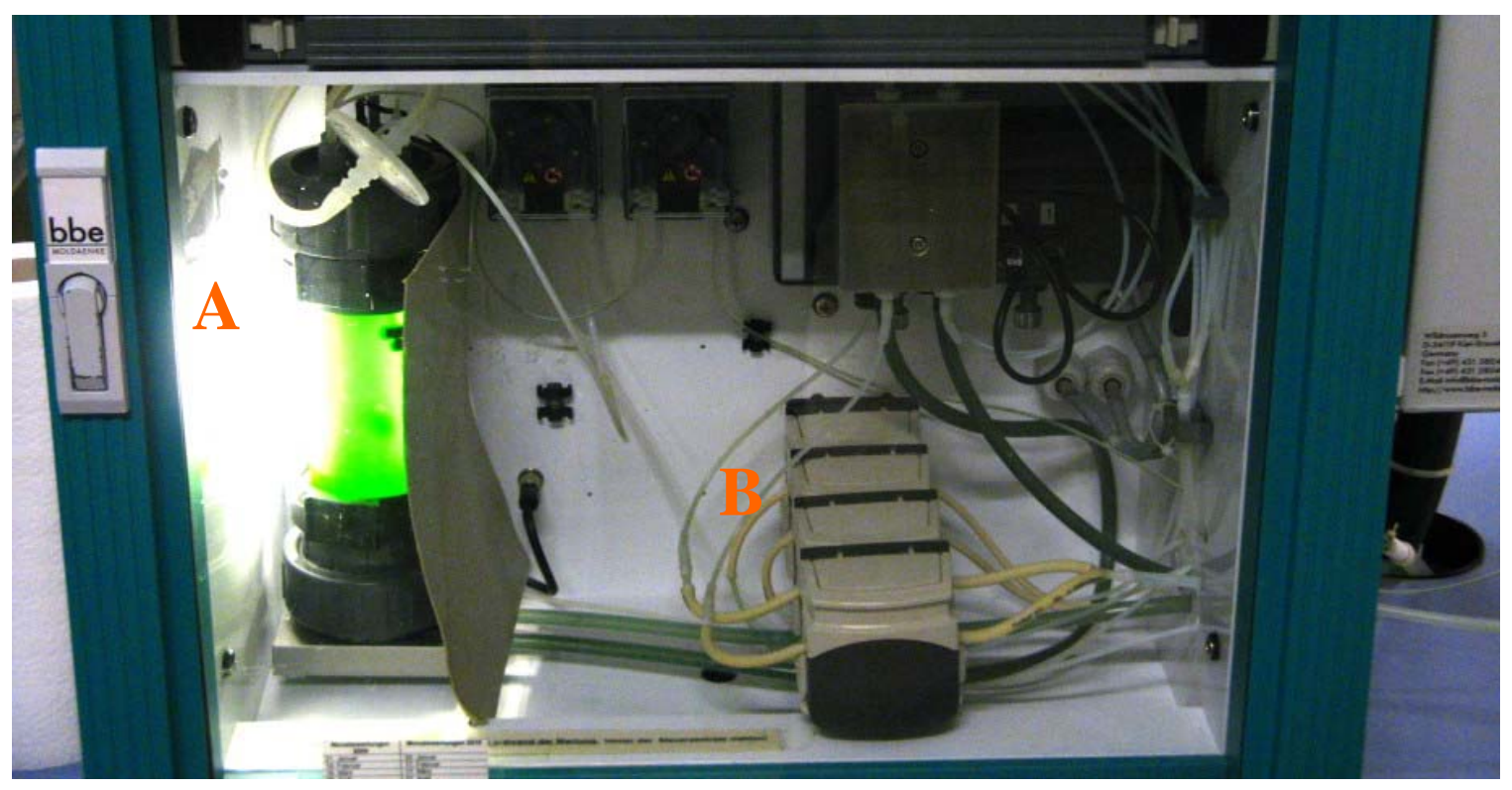

Figure 3.24. The bbe Daphnia Toximeter in use at the Hardhof groundwater plant in Zurich, Switzerland, showing: A) the alage fermenter for automatic feeding of D. magna; and $\mathrm{B}$ ) the observation chambers containing organisms. 
This system has worked appropriately for the past four years and is an example about how an early warning system can be incorporated successfully into a drinking water monitoring system. Comparisons will be drawn between this system and the automated systems investigated in this study to determine the potential success of implementation of these systems in Ontario.

\subsection{Importance of implementation of an early-warning system for monitoring drinking water}

On June 7, 2010, large amounts of toxic waste from a copper mine in the Shanghang county in China leaked into the Tingjiang river, destroying almost 2000 tons of fish in fisheries along the river. The spill was only confirmed and warning given to residents and fish farmers on July 12, 2010, partly due to the perception that copper is not very toxic. As stated by the Zijin Mining Group responsible for the spill, the sewage released "contained copper, but no extremely toxic substances" (Tian et al., 2010). This perception that copper is not very toxic may leave copper spills particularly susceptible to underreporting as was the case in China. Many fish farmers lost their livelihoods, and some have stated that if they "had been told earlier, we would have transferred the fish to safe ponds and saved a lot of money", which stresses the importance of having early warning systems in place. The Shanghang province also relies on the Tingjiang river for providing its residents with tap water, but no ill effects on residents have been noted, as it is reported that they have not used the tap water for drinking since the mine opened in 1993 (Tian et al., 2010).

Events like these are not limited to China. The real-time early-warning automated system that this study is contributing to is to be implemented in the Niagara region in Southern Ontario, particularly in a water distribution facility that takes water from the Welland Canal. As recently as June 15, 2010, a diesel spill in the Welland Canal led Port Colborne officials to advise residents not to consume or use drinking water for 24 hours after the spill (Dunning, 2010). The warning was not based on detection of contaminant but simply as a precaution following the spill. Residents were warned to not use the water 
for drinking, bathing, cooking, or washing fruits and vegetables (Dunning, 2010). This incident highlights the fact that residents of the Niagara region are still greatly affected by occasional spills in the Welland Canal, which makes it particularly useful to implement an early-warning system in this region. Had such a system been in place, it would detect the presence of the substance, and a water advisory would have only been put forth if the early-warning system had triggered an alarm. In the event that no water contamination was actually detected, then it is possible that residents of Port Colborne would not have been as inconvenienced by a precautionary water advisory.

\subsection{Suitability of Brachionus spp. as test organisms}

The rotifers Brachionus calyciflorus and Brachionus havanensis were assessed for potential use in an image analysis system, with the potential of using cultures with the ECOTOX system. Organisms were assessed by: ease of culturing, size, culture quality and density, and reaction to contaminants. Cultures were obtained from A. Derry at McGill University in Montreal, QC, where they are grown in mixed-culture chemostats together with the food source, Chlorella vulgaris. The option of growing these organisms in chemostats was not available at Ryerson University, and these organisms were grown in batch cultures instead.

The medium and setup for the batch cultures is a straightforward process; however, there was some difficulty in maintaining these cultures, as there tended to be large cycles of Chlorella blooms where Brachionus densities were very low, and algae decline and near crashes of Brachionus cultures. Observation under the microscope indicated that under high densities of Chlorella, there was difficulty of movement seen in Brachionus. Under these instances this would also negatively affect an attempt at detecting Brachionus behaviour, not only because it is impeded but also because the high density of algae would disrupt the image analysis as well. It would appear that maintaining batch cultures of Brachionus spp. would require daily monitoring and 
adjusting of algae levels, and this would make it less ideal as a test organism unless chemostats are available.

Preliminary observation under the microscope showed that both species of Brachionus reacted immediately to addition of $200 \mu \mathrm{M}$ of copper, showing complete loss of motility in under one minute. This indicates that these species are sensitive to copper as a contaminant. Despite this, they could not be used with the ECOTOX system due to an incompatibility in size. The size of B. calyciflorus is in the range of $250-300 \mu \mathrm{m}$ (A. Derry, personal communication, January 2010), and after compared to the size of $E$. gracilis under the microscope it was determined that this species is too big for the observation cuvette in ECOTOX. B. havanensis, in contrast, is smaller, at 200-250 $\mu \mathrm{m}$ (A. Derry, personal communication, January 2010). Culture densities, however, did not rise above 2000 individuals per $\mathrm{mL}$, which is not compatible with the image analysis principle of ECOTOX, where it would be unlikely that in an observation area of less than $1 \mathrm{~mm}^{2}$ one would see individual Brachionus frequently enough to be able to measure swimming behaviour efficiently. Due to their unique sizing, if Brachionus spp. are to be used in behavioural image analysis for toxicity testing, a system must be specifically designed for these species' size and density. Currently they are therefore not recommended to be used with automated systems of image analysis of behaviour.

\subsection{Conclusion and recommendations}

E. gracilis is a sensitive organism, especially to copper. It has the potential to have high sensitivities for other metals as well, and its behaviour must be therefore investigated after exposure to other metals of concern such as mercury and cadmium. While visual observation of cell shape was found to be sensitive to copper, visual observation of cell motility was in fact a much more sensitive parameter, producing $\mathrm{EC}_{50} \mathrm{~S}$ at $21^{\circ} \mathrm{C}$ of $1.389 \mu \mathrm{M}$ after $1 \mathrm{~h}$ incubation and $1.529 \mu \mathrm{M}$ after $2 \mathrm{~h}$, and at $26^{\circ} \mathrm{C} \mathrm{EC}_{50} \mathrm{~S}$ of $0.947 \mu \mathrm{M}$ after $1 \mathrm{~h}$ and $0.818 \mu \mathrm{M}$ after $2 \mathrm{~h}$, indicating higher sensitivity at higher temperatures. Cell motility was found to recover after $24 \mathrm{~h}$, and further research is needed 
to track the recovery process of the cells throughout the $24 \mathrm{~h}$ and investigate the process under which this is happening. The visual observations of motility did not detect any changes after exposure to atrazine and TBT, which could make E. gracilis a good discriminating organism for differentiating contamination by metals and organic contaminants.

The image analysis system ECOTOX was less sensitive than all visual observations for copper. However, the system was more sensitive for atrazine and TBT, having detected changes in gravitactic behaviour after $24 \mathrm{~h}$ incubation in these contaminants. This indicates that the ECOTOX system has the potential for being a sensitive automated system for the detection of these organic contaminants in an early warning system for drinking water quality. A modification of the system to improve measures of cell motility and/or swimming velocity, such as including a horizontally paced cuvette, would greatly improve the suitability of this system for use in a drinking water quality testing setting.

The DaphniaTox system is not applicable at its present state due to software instability issues; however, D. magna's phototactic behaviour, a parameter measured by DaphniaTox, has the potential for being a highly sensitive behavioural parameter, and more studies are needed to observe this behaviour under exposure to different contaminants.

The rotifer species examined, B. calyciflorus and B. havanensis, were not considered suitable at this time. The ECOTOX automated image analysis system was previously thought to be able to accommodate these species' particular size and culture densities, but this study concluded that it cannot at this time. It does, however, have the potential to be modified to include a larger observation chamber and a lesser lens magnification, and become very suitable for inclusion in this system.

Finally, many modifications are needed to the ECOTOX system if it is to be implemented in a water quality testing facility with minimal supervision, such as the inclusion of a chemostat as is the case with the bbe Daphnia Toximeter. Because the system detected changes in the organic contaminants tested after $24 \mathrm{~h}$ incubation, it is 
recommended that changes to the system incorporate $24 \mathrm{~h}$ incubation in water samples being tested to allow for a visible effect. Automated measurements should be set to hourly to include more immediate effects such as was seen with copper, which are not apparent after $24 \mathrm{~h}$ incubation. Considering the sensitivity of E. gracilis, with these changes and with increased technical support ECOTOX would be a suitable image analysis system for implementation in an early warning system for drinking water quality. 


\section{References}

Adams, S.M. \& Greeley, M.S. (2000). Ecotoxicological indicators of water quality: using multi-response indicators to assess the health of aquatic ecosystems. Water, Air, and Soil Pollution, 123, 103-115.

Ahmed, H., \& Häder, D.-P. (2010a). A fast algal bioassay for assessment of copper toxicity in water using Euglena gracilis. Journal of Applied Phycology, 1, 1-8.

Ahmed, H., \& Häder, D.-P. (2010b). Rapid ecotoxicological bioassay of nickel and cadmium using motility and photosynthetic parameters of Euglena gracilis. Environmental and Experimental Botany, 69, 68-75.

Allan, I. J., Vrana, B., Greenwood, R., Mills, G. A., Roig, B., \& Gonzalez, C. (2006). A "toolbox" for biological and chemical monitoring requirements for the European Union's water framework directive. Talanta, 69, 302-322.

Alzieu, C. (1998). Tributyltin: case study of a chronic contaminant in the coastal environment. Ocean and Coastal Management, 40, 23-36.

Anderson, T.D. \& Zhu, K.Y. (2004). Synergistic and antagonistic effects of atrazine on the toxicity of organophosporodithioate and organophosphorotioate insecticides to Chironomus tentans (Diptera: Chironomidae). Pesticide Biochemistry and Physiology, 80, 54-64.

Baillieul, M. \& Scheunders, P. (1998). On-line determination of the velocity of simultanesously moving organisms by image analysis for the detection of sublethal toxicity. Water Research, 32(4), 1027-1034.

Barata, C., Baird, D.J., Nogueira, A.J.A, Soares, A.M.V.M., \& Riva, M.C. (2006). Toxicity of binary mixtures of metals and pyrethroid insecticides to Daphnia magna Straus. Implications for multi-substance risks assessment. Aquatic Toxicology, 78, 1-14.

Bartlett, A.J., Borgmann, U., Dixon, D.G., Batchelor, S.P., \& Maguire, R.J. (2005). Toxicity and bioaccumulation of tributyltin in Hyalella azteca from freshwater harbour sediments in the Great Lakes Basin, Canada. Canadian Journal of Fisheries and Aquatic Sciences, 62, 1243-1253.

BBE Moldaenke. (n.d.). Daphnia Toximeter II: A powerful instrument for water toxicity assessment. Accessed on July 10, 2010, from http://www.bbe-moldaenke.de/ fileadmin/Documents/Brochures/Daphnia_Toximeter.pdf

Beitinger, T. (1990) Behavioural reactions for the assessment of stress in fish. Journal of Great Lakes Research, 16, 495-528. 
Bérard, A. (1996). Effect of four organic solvents on natural phytoplankton assemblages: consequences for ecotoxicological experiments on herbicides. Bulletin of Environmental Contamination \& Toxicology, 57, 183-190.

Bitton, G., \& Koopman, B. (1992). Bacterial and enzymatic bioassays for toxicity testing in the environment. Reviews of Environmental Contamination \& Toxicology, 125, $1-22$.

Bogue, M.B. (2000). Fishing the Great Lakes: an environmental history, 1783-1933. Madison, WI: University of Wisconsin Press.

Borgmann, U., Chau, Y.K., Wong, P.T.S., Brown, M. \& Yaromich, J. (1996). The relationship between tributyltin (TBT) accumulation and toxicity to Hyalella azteca for use in identifying TBT toxicity in the field. Journal of Aquatic Ecosystem Health, 5, 199-206.

Bowman, M.C., Oller, W.L., \& Cairns, T. (1981). Stressed bioassay systems for rapid screening of pesticide residues. Part I: evaluation of bioassay systems. Archives of Environmental Contamination \& Toxicology, 10, 9-24.

Bunn, S.E. (1995) Biological monitoring of water quality in Australia: Workshop summary and future directions. Australian Journal of Ecology, 20, 220-227.

Butterworth, F.M., Gunatilaka, A., \& Gonsebatt, M.E. (2001). Biomonitors and biomarkers as indicators of environmental change 2: a handbook. New York, NY: Kluwer Academic/Plenum Publishers.

Cairns, J., Jr. \& Mount, D.I. (1990). Aquatic Toxicology. Environmental Science \& Technology, 24(2), 154-161.

Castillo, L.E., Pinnock, M., \& Martinez, E. (2000). Evaluation of a battery of toxicity tests for use in the assessment of water quality in a Costa Rican laboratory. Environmental Toxicology, 15(4), 312-321.

Charoy, C. \& Janssen, C. (1999). The swimming behaviour of Brachionus calyciflorus (rotifer) under toxic stress. II. Comparative sensitivity of various behavioural criteria. Chemosphere, 38(14), 3247-3260.

Chau, Y.K., Maguire, R.J., Brown, M., Yang, F., \& Batchelor, S.P. (1997). Occurrence of organotin compounds in the Canadian aquatic environment five years after the regulation of antifouling uses of tributyltin. Water Quality Research Journal of Canada, 32, 453-521.

Checcucci, A. (1976). Molecular sensory physiology of Euglena. Naturwissenschaften, 63, 412-417. 
Checcucci, A., Colombetti, G., Ferrara, R., \& Lenci, F. (1976). Action spectra for photoaccumulation of green and colorless Euglena gracilis: Evidence for Identification of receptor Pigments. Photochemistry and Photobiology, 23, 51-54.

Christensen, B.T., Lauridsen, T.L., Ravn, H.W., \& Bayley, M. (2005). A comparison of feeding efficiency and swimming ability of Daphnia magna exposed to cypermethrin. Aquatic Toxicology, 73, 210-220.

City of Niagara Falls. (2010). Manufacturing. Accessed on July 22, 2010, from http://www.niagarafalls.ca/business/business_profile/manufacturing.asp

City of Port Colborne (2010). Drinking Water System. Retrieved on July 21, 2010, from http://www.pcetdc.ca/page/Water_Quality.

City of St. Catharines. (2010). Water Distribution System 2009 Summary Report. St. Catharines, Waterworks \#260003279.

City of Welland. (2008). City of Welland Drinking Water Quality Assurance Program. Accessed on July 21, 2010, from http://www.welland.ca/Eng/WaterReports.asp.

Clarke, S.M., Barrick, C.W., \& Samoiloff, M.R. (1990). A Bioassessment battery for use in an industrial setting: A new management approach. Toxicity Assessment, 5(2), 153-166.

Connell, D.W. (1995). Prediction of bioconcentration and related lethal and sublethal effects with aquatic organisms. Marine Pollution Bulletin, 31, 201-205.

Daiker, V., Lebert, M., Richter, P., \& Häder, D.-P. (2010). Molecular characterization of a calmodulin involved in the signal transduction chain of gravitaxis in Euglena gracilis. Planta, 231, 1229-1236.

Danilov, R.A. \& Ekelund, N.G.A. (2001a). Applicability of growth rate, cell shape, and motility of Euglena gracilis as physiological parameters for bioassessment at lower concentrations of toxic substances: an experimental approach.

Environmental Toxicology, 16(1), 78-83.

Danilov, R.A. \& Ekelund, N.G.A. (2001b). Responses of photosynthetic efficiency, cell shape and motility in Euglena gracilis (euglenophyceae) to short-term exposure to heavy metals and pentachlorophenol. Water, Air, \& Soil Pollution, 132, 61-73.

de Bisthoven, L.J., Gerhardt, A., \& Soares, A. M.V.M. (2004). Effects of acid mine drainage on larval Chironomus (diptera, chironomidae), measured with the Multispecies Freshwater Biomontor ${ }^{\circledR}$. Environmental Toxicology and Chemistry, 23(5), 1123-1128. 
de Kuhn, R.M., Streb, C., Breiter, R., Richter, P., Neesse, T., \& Häder, D.-P. (2006). Screening for unicellular algae as possible bioassay organisms for monitoring marine water samples. Water Research, 40, 2695-2703.

de Schamphelaere, K.A.C. \& Janssen, C.R. (2002). A biotic ligand model predicting acute copper toxicity for Daphnia magna: the effects of calcium, magnesium, sodium, potassium, and pH. Environmental Science \& Technology, 36, 48-54.

Denoyelles, F., Kettle, W.D. \& Sinn, D.E. (1982). The responses of plankton communities in experimental ponds to atrazine, the most heavily used pesticide in the United States. Ecology, 63, 1285-1293.

Detenbeck, N.E., Hermanutz, R., Allen, K. \& Swift, M. (1996). Fate and effects of the herbicide atrazine in flow-through wetland mesocosms. Environmental Toxicology and Chemistry, 14, 937-946.

Dewey, S. (1986). Effects of the herbicide atrazine on aquatic insect community structure and emergence. Ecology, 67, 148-162.

Dodson, S.I. \& Hanazato, T. (1995). Commentary on effects of anthropogenic and natural organic chemicals on development, swimming behavior, and reproduction of Daphnia, a key member of aquatic ecosystems. Environmental Health Perspectives, 103(Suppl 4), 7-11.

Dort, A. (2010). The Application of an Early-warning Biomonitoring System (EWBS) in a Canadian Context. Master's Thesis, Toronto, Ryerson University.

Doving, K. (1991) Assessment of animal behaviour as a method to indicate environmental toxicity. Comparative Biochemistry and Physiology, 1/2, 247-252.

Ducrot, V., Usseglio-Polatera, P., Péry, A.R.R., Mouthon, J., Lafont, M., Roger, M.-C., Garric, J., \& Férard, J.-F. (2005). Using aquatic macroinvertebrate species traits to build test batteries for sediment toxicity assessment: Accounting for the diversity of potential biological responses to toxicants. Environmental Toxicology and Chemistry, 24(9), 2306-2315.

Dunning, J. (2010, June 15). Port Colborne residents told not to drink water - Diesel spill in Welland Canal spawns precautionary measures. The Hamilton Spectator. Retrieved July 20, 2010, from http://www.thespec.com/News/BreakingNews/ article/789567

Durand, A.M., Rotteveel, S., Collombon, M.T., van der Grinten, E., Maas, J.L., \& Verweij, W. (2009). Toxicity measurements in concentrated water samples. Bilthoven, the Netherlands: RIVM Report 607013010/2009. Accessed on April 12, 2010, from http:/www.rivm.nl/bibliotheek/rapporten/607013010.pdf 
Einicker-Lamas, M., Mezian, G.A., Fernandes, T.B., Silva, F.L.S., Guerra, F., Miranda, K., Attias, M., \& Oliveira, M.M. (2002). Euglena gracilis as a model for the study of $\mathrm{Cu}^{2+}$ and $\mathrm{Zn}^{2+}$ toxicity and accumulation in eukaryotic cells. Environmental Pollution, 120, 779-786.

Environment Canada (1996). Biological Test Method: Acute Lethality Test Using Daphnia spp.

Environment Canada (2000). Biological test method: Reference method for determining acute lethality of effluents to Daphnia magna. $2^{\text {nd }}$ ed. Ottawa: Minister of Public Works and Government Services.

Environment Canada (2007). Biological Test Method: Growth Inhibition Test Using Freshwater Alga. Environmental Science and Technology Centre, Science and Technology Branch. EPS 1/RM/25 $2^{\text {nd }}$ edition. Ottawa: Minister of Public Works and Government Services.

Erickson, R.J., Benoit, D.A., Mattson, V.R., Nelson, H.P.,Jr., \& Leonard, E.N. (1996). The effects of water chemistry on the toxicity of copper to fathead minnows. Environmental Toxicology and Chemistry, 15(2), 181-193.

Ernst, W.H.O. (1997). Population dynamics of plants under exposure and the selection of resistance. In G. Schüürmann \& B. Markert (Eds.), Ecotoxicology: ecological fundamentals, chemical exposure, and biological effects. (pp. 117-132). New York, NY: John Wiley \& Sons, Inc.

Fernandez-Casalderrey, A., Ferrando, M.D., \& Andreu-Moliner, E. (1994). Effect of sublethal concentrations of pesticides on the feeding behavior of Daphnia magna. Ecotoxicology and Environmental Safety, 27, 82-89.

Findlay, R. \& Telford, P. (2006). The International Joint Commission and the Great lakes Water Quality Agreement: lessons for Canada-United States Regulatory Co-operation. Government of Canada Working Paper Series 023.

Flagship Ventures (2009). Petrel Biosensors. Accessed on July 10, 2010, from http://habcam.whoi.edu/HabCamData/powerpoints\&posters/Petrel.M2.final_SMG .ppt

Flemming, C.A., \& Trevors, J.T. (1989). Copper toxicity and chemistry in the environment: A review. Water, Air, and Soil Pollution, 44, 143-158.

Flickinger, A.L., Randall, J.F., Winner, R.W., \& Skillings, J.H. (1982). Filtration and phototactic behavior as indices of chronic copper stress in Daphnia magna Straus. Archives of Environmental Contamination and Toxicology, 11, 457-463. 
Fochtman, P., Raszka, A., \& Nierzedska, E. (2000). The use of conventional bioassays, microbiotests, and some "rapid" methods in the selection of an optimal test battery for the assessment of pesticides toxicity. Environmental Toxicology, 15(5), 376-384.

Forbes, V.E., Palmqvist, A., \& Bach, L. (2006). The use and misuse of biomarkers in ecotoxicology. Environmental Toxicology and Chemistry, 25, 272-280.

Gerhardt, A. (2007). Importance of xposure route for behavioural responses in Lumbriculus variegatus Müller (oligochaeta: lumbriculida) in short-term exposures to Pb. Environmental Science and Pollution Research, 14(6), 430-434.

Gerhardt, A. (2009). Screening the toxicity of $\mathrm{Ni}, \mathrm{Cd}, \mathrm{Cu}$, ivermectin, and imidacloprid in a short-term automated behavioural toxicity test with Tubifex tubifex (Muller 1774) (oligochaeta). Human and Ecological Risk Assessment, 15, 27-40.

Gerhardt, A., Bisthoven, L.J. \& Schmidt, S. (2006a). Automated recording of vertical negative phototactic behaviour in Daphnia magna Straus (Crustacea). Hydrobiologia, 559, 433-441.

Gerhardt, A., Ingram, M.K., Kang, I.J. \& Ulitzur, S. (2006b). In situ on-line toxicity biomonitoring in water: recent developments. Environmental Toxicology and Chemistry, 25, 2263-2271.

Giesy, J.P. \& Hoke, R.A. (1989). Freshwater sediment toxicity and bioassessment: Rationale for species selection and test design. Journal of Great Lakes Research, 15(4), 539-569.

Girling, A.E., Pascoe, D., Janssen, C.R., Peither, A., Wenzel, A., Schäfer, H., Neumeier, B., Mitchell, G.C., Taylor, E.J., Maund, S.J., Lay, J.P., Jüttner, I., Crossland, N.O., Stephenson, R.R., \& Persoone, G. (2000). Development of methods for evaluating toxicity to freshwater ecosystems. Ecotoxicology and Environmental Safety, 45, 148-176.

Goodrich, M.S. \& Lech, J.J. (1990). A behavioral screening assay of Daphnia magna: a method to assess the effects of xenobiotics on spatial orientation. Environmental Toxicology and Chemistry, 9, 21-30.

Government of Canada. (2009, August 8). Proposed risk management approach for nonpesticidal organotin compunds. Prepared for Environment Canada \& Health Canada.

Government of Ontario (2010). Protocol of Accepted Drinking Water Testing Methods, Version 2.0. Ontario: Laboratory Services Brach, Ministry of the Environment. 
Grand, I., Schildknecht, A., \& Köster, O. (n.d.) Wasserflöhe in der Wasserversorgung [Water fleas in the water supply]. Zurich: Wasserversorgung Zürich.

Graymore, M., Stagnitti, F. \& Allinson, G. (2001). Impacts of atrazine in aquatic ecosystems. Environment International, 26, 483-495.

Green, U., Kremer, J.H., Zillmer, M. \& Moldaenke, C. (2003) Detection of chemical threat agents in drinking water by an early warning real-time biomonitor. Environmental Toxicology, 18, 368-374.

Gullick, R.W., Grayman, W.M., Deininger, R.A. \& Males, R.M. (2003). Design of earlywarning monitoring systems for source waters. Journal of the American Water Works Association, 95(11), 58-72.

Haap, T., Triebskorn, R., \& Kohler, H.-R. (2007). Acute effects of diclofenac and DMSO to Daphnia magna: Immobilisation and hsp70-induction. Chemosphere, 73(3), 353-359.

Häder, D.-P. (n.d.) ECOTOX Version 5.0 Real Time Computer Automatic Toxicity Monitoring Manual. Moehrendorf, Germany.

Häder, D.-P., Lebert, M., Richter, P., \& Ntefidou, M. (2003). Gravitaxis and graviperception in flagellates. Advances in Space Research, 31(10), 2181-2186.

Hatch, A.C. \& Burton, G.A. (1999). Photo-induced toxicity of PAHs to Hyalella azteca and Chironomus tentans: effects of mixtures and behavior. Environmental Pollution, 106, 157-167.

Health Canada. (1992). Copper. Retrieved on July 22, 2010, from: http://www.hcsc.gc.ca/ewh-semt/pubs/water-eau/copper-cuivre/index-eng.php

Health Canada (1993) Atrazine. Retrieved on July 22, 2010, from: http:/www.hcsc.gc.ca/ewh-semt/pubs/water-eau/atrazine/index-eng.php.

Hermens, J., Canton, H., Janssen, P. \& de Jong, R. (1984). Quantitative structure-activity relationships and toxicity studies of mixtures of chemicals with anaesthetic potency: acute lethal and sublethal toxicity to Daphnia magna. Aquatic Toxicology, 5, 143-154.

Huang, L., Xi, Y.-L., Zha, C.-W., \& Zhao, L.-L. (2007). Effect of aldrin on life history characteristics of rotifer Brachionus calyciflorus Pallas. Bulletin of Environmental Contamination \& Toxicology, 79, 524-528.

IARC (1999). Atrazine. IARC Monographs Volume 73. Retrieved on July 22, 2010, from http://monographs.iarc.fr/ENG/Monographs/vol73/mono73-8.pdf 
International Joint Commission. (2006). Report on Spills in the Great Lakes Basin with a Special Focus on the St. Clair-Detroit River Corridor. Retrieved on July 23, 2010, from http://www.ijc.org/php/publications/pdf/ID1594.pdf.

International Joint Commission (IJC), 2009. Great Lakes Water Quality Agreement Priorities 2007-09 Series. Work Group Report on Great Lakes Chemicals of Emerging Concern, 2009. IJC, Special Publication 2009-01, Windsor, Ontario, Canada.

Iseki, M., Matsunaga, S., Murakami, A., Ohno, K., Shiga, K., Yoshida, K., Sugai, M., Takahashi, T., Hori, T., \& Watanabe, M. (2002). A blue-light-activated adenylyl cyclase mediates photoavoidance in Euglena gracilis. Nature, 415, 1047-1051.

Isidori, M., Lavorgna, M., Nardelli, A., \& Parrella, A. (2003). Toxicity identification evaluation of leachates from municipal solid waste landfills: a multispecies approach. Chemosphere, 52, 85-94.

Jeon, J., Kim, J.H., Lee, B.C., \& Kim, S.D. (2008). Development of a new biomonitoring method to detect the abnormal activity of Daphnia magna using automated Grid Counter device. Science of the Total Environment, 389, 545-556.

Katsumata, M., Koike, T., Nishikawa, M., Kazumura, K., \& Tsuchiya, H. (2006). Rapid ecotoxicological bioassay using delayed fluorescence in the green alga Pseudokirchneriella subcapitata. Water Research, 40, 3393-3400.

Kettrup, A. \& Marth, P. (1998). Specimen banking as an environmental surveillance tool. In G. Schüürmann \& B. Markert (Eds.), Ecotoxicology: Ecological Fundamentals, Chemical Exposure, and Biological Effects. (pp. 413-436). New York: John Wiley \& Sons, Inc.

Kieu, N.D., Michels, E. \& de Meester, L. (2001) Phototactic behavior of Daphnia and the continuous monitoring of water quality: interference of fish kairomones and food quality. Environmental Toxicology and Chemistry, 20, 1098-1103.

Knauert, S. \& Knauer, K. (2008). The role of reactive oxygen species in copper toxicity to two freshwater green algae. Journal of Phycology, 44, 311-319.

Konstantinou, I.K. \& Albanis, T.A. (2004). Worldwide occurrence and effects of antifouling paint booster biocides in the aquatic environment: a review. Environment International, 30, 235-248.

Lachney, C.L. \& Lonergan, T.A. (1985). Regulation in cell shape of Euglena gracilis. III. involvement of stable microtubules. Journal of Cell Science, 74, 219-237.

Landis, W.G. \& Yu, M.-H. (1995). Environmental Toxicology: Impacts of Chemicals Upon Ecological Systems. Boca Raton, FL: CRC Press. 
Laval-Martin, D., Grizeau, D., \& Calvayrac, R. (1983). Characterization of diuronresistant Euglena: greater tolerance for various phenylurea herbicides and increased sensitivity of thylakoids to ethyl-s-dipropyl thiocarbamate. Plant Science Letters, 29, 155-167.

LAWA 'Biomonitoring' Committee (1996). Recommendation on the Deployment of Continuous Biomonitors for the Monitoring of Surface Waters. Berlin: Working Group of the Federal States on Water Problems (LAWA).

Lazorko-Connon, S. \& Achari, G. (2009). Atrazine: its occurrence and treatment in water. Environmental Review, 17, 199-241.

Lebert, M. \& Häder, D.-P. (1999). Negative gravitactic behavior of Euglena gracilis can not be described by the mechanism of buoyancy-oriented upward swimming. Advances in Space Research, 24(6), 851-860.

LeBlanc, G.A. \& Surprenant, D.C. (1983). The acute and chronic toxicity of acetone, dimethyl formamide, and triethylene glycol to Daphnia magna (Straus). Archives of Environmental Contamination \& Toxicology, 12, 305-310.

Lechelt, M., Blohm, W., Kirschneit, B., Pfeiffer, M., Gresens, E., Liley, J., Holz, R., Luering, C., \& Moldaenke, C. (2006). Monitoring of surface water by ultrasensitive Daphnia toximeter. Environmental Toxicology, 15, 390-400.

Liu, Z. T., Kong, Z.M., Zhou, F., \& Wang, L.S. (1996). Bioconcentration and toxicity effect on lipid content of aquatic organisms. Bulletin of Environmental Contamination and Toxicology, 56, 135-142.

Lockert, C.K., Hoagland, K.D., \& Siegfried, B.D. (2006). Comparative sensitivity of freshwater algae to atrazine. Bulletin of Environmental Contamination and Toxicology, 76, 73-79.

Maltby, L., Clayton, S.A., Wood, R.M., \& McLoughlin, N. (2001). Evaluation of the Gammarus pulex in situ feeding assay as a biomonitor of water quality: robustness, responsiveness, and relevance. Environmental Toxicology and Chemistry, 21, 361-368.

Marshall, G.C. (2009). Assessing behavioural and physiological responses of three aquatic invertebrates to atrazine and tributyltin in a multi-species, early-warning biomonitoring technology. Master's Thesis, Toronto, Ryerson University.

Martins, J., Soares, M.L., Saker, M.L., OlivaTeles, L., \& Vasconcelos, V.M. (2007). Phototactic behavior in Daphnia magna Straus as an indicator of toxicants in the aquatic environment. Ecotoxicology and Environmental Safety, 67, 417-422. 
Maxwell, K. \& Johnson, G.N. (2000). Chlorophyll fluorescence - a practical guide. Journal of Experimental Botany, 51(345), 659-668.

McCarthy, L.H. (2007) NSERC Strategic Grant Proposal.

Michels, E., Leynen, M., Cousyn, C., de Meester, L. \& Ollevier, F. (1999). Phototactic behaviour of Daphnia as a tool in the continuous monitoring of water quality: Experiments with a positively phototactic Daphnia magna clone. Water Resources, 33(2), 401-408.

Michels, E., Semsari, S., Bin, C. \& de Meester, L. (2000). Effect of sublethal doses of cadmium on the phototactic behavior of Daphnia magna. Ecotoxicology and Environmental Safety, 47, 261-265.

Mikol, Y.B., Richardson, W.R., van der Schalie, W.H., Shedd, T.R. \& Widder, M.W. (2007). An online real-time biomonitor for contaminant surveillance in water supplies. Journal of the American Water Works Association, 99, 107-116.

Miller, W.E., Peterson, S.A., Greene, J.C., \& Callahan, C.A. (1985). Comparative toxicology of laboratory organisms for assessing hazardous waste sites. Journal of Environmental Quality, 14(4), 569-574.

Moore, M.T., Lizotte, R.T., Knight, S.S., Smith, S. \& Cooper, C.M. (2006). Assessment of pesticide contamination in three Mississippi Delta oxbow lakes using Hyalella azteca. Chemosphere, 67, 2184-2191.

Moore, R.B. (1970). Effects of pesticides on growth and survival of Euglena gracilis Z. Bulletin of Environmental Contamination \& Toxicology, 5(3), 226-230.

National Research Council of the United States \& The Royal Society of Canada. (1985). The Great lakes Water Quality Agreement: an evolving instrument for ecosystem management. Washington, D.C.: National Academy Press.

Navarro, L., Torres-Márquez, M.E., González-Moreno, S., Devars, S., Hernández, R., \& Moreno-Sánchez, R. (1997). Comparison of physiological changes in Euglena gracilis during exposure to heavy metals of heterotrophic and autotrophic cells. Comparative Biochemistry and Physiology, 166C(3), 265-272.

Nebeker, A.V., Cairns, M.A., Gakstatter, J.H., Malueg, K.W., Schuytema, G.S., \& Krawczyk, D.F. (1984). Biological methods for determining toxicity of contaminated freshwater sediments to invertebrates. Environmental Toxicology and Chemistry, 3(4), 617-630.

Newman, M.C. \& Clements, W.H. (2008). Ecotoxicology: A Comprehensive Treatment. Boca Raton, FL: CRC Press, Taylor \& Francis Group. 
Niagara Region. (2010). Agricultural Planning. Retrieved on July 21, 2010, from http://www.niagararegion.ca/living/ap/default.aspx

NPCA - Niagara Peninsula Conservation Authority. (2010, April). NPCA Water Quality Monitoring Program: 2009 Annual Report. Retrieved on July 21, 2010, from http://www.npca.ca/about/minutes/May19-10meeting-agenda.pdf.

Oberdörster, E., Rittschof, D. \& LeBlanc, G.A. (1998). Alteration of $\left[{ }^{14} \mathrm{C}\right]$-testosterone metabolism after chronic exposure of Daphnia magna to tributyltin. Archives of Environmental Contamination and Toxicology, 34, 21-35.

O'Connor, D. R. (2002). Report of the Walkerton Inquiry: A Strategy for Safe Drinking Water. Toronto: Ontario Ministry of the Attorney General.

OECD. (2004). OECD Guideline for testing of chemicals: Daphnia sp., Acute Immobilization Test. Paris, France.

Ohta, M. \& Suzuki, T. (2007). Participation of the inositol phospholipid signaling pathway in the increase in cytosolic calcium induced by tributyltin chloride intoxication of chlorophyllous protozoa Euglena gracilis Z and its achlorophyllous mutant SM-ZK. Comparative Biochemistry and Physiology, part C, 146, 525-530.

Ontario Ministry of the Environment (2001). Ontario Drinking Water Standards.

Passarelli, V., Barsanti, L., \& Gualtieri, P. (1990). A simple device for ultramicroscopic studies of motile microorganisms. Micron and Microscopica Acta, 21, 101-104.

Pearce, C.J.E. (2009). Investigating the Sub-acute Responses of Lemna minor, Pseudokirchneriella subcapitata, Euglena gracilis and Anodonta grandis to tributyltin-hydride and atrazine in the Development of an Early-warning Biomonitoring System to Rapidly Detect Source-Water Contaminants. Master's Thesis, Toronto, Ryerson University.

Pelaez, M., Antoniou, M.G., He, X., Dionysiou, D.D., de la Cruz, A.A., Tsimeli, K., Triantis, T., Hiskia, A., Kaloudis, T., Williams, C., Aubel, M., Chapman, A., Foss, A., Khan, U., O'Shea, K.E., \& Westrick, J. (2010). Sources and occurrence of cyanotoxins worldwide. In Fatta-Kassinos, D., Bester, K., \& Kümmerer, K. (Eds.), Xenobiotics in the Urban Water Cycle: Mass Flows, Environmental Processes, Mitigation and Treatment Strategies. New York: Springer.

Pettersson, M. \& Ekelund, N.G.A. (2006). Effects of the herbicides roundup and avans on Euglena gracilis. Archives of Environmental Contamination and Toxicology, 50, 175-181. 
Ren, Z., Li, Z., Ma, M., Wang, Z., \& Fu, R. (2009). Behavioral responses of Daphnia magna to stresses of chemicals with different toxic characteristics. Bulletin of Environmental Contamination and Toxicology, 82, 310-316.

Richter, P., Börnig, A., Streb, C., Ntefidou, M., Lebert, M., \& Häder, D.-P. (2003). Effects of increased salinity of gravitaxis in Euglena gracilis. Journal of Plant Physiology, 160, 651-656.

Richter, P.R., Lebert, M., Tahedl, H., \& Häder, D.-P. (2001). Physiological characterization of gravitaxis in Euglena gracilis and Astasia longa studied on sounding rocket flights. Advances in Space Research, 27(5), 983-988.

Richter, P.R., Schuster, M., Lebert, M., Streb, C., \& Häder, D.-P. (2007). Gravitaxis of Euglena gracilis depends only partially on passive buoyancy. Advances in Space Research, 39, 1218-1224.

Rochetta, I., Ruiz, L.B., Rios de Molina, M.C., \& Conforti, V. (2006). Chromium toxicity to Euglena gracilis strains depending on the physicochemical properties of the culture medium. Bulletin of Environmental Contamination and Toxicology, 76, $512-521$.

Roig, B., Allan, I.J., \& Greenwood, R. (2003). A Toolbox of existing and emerging methods for water monitoring under the WFD. SWIFT-WFD, Screening methods for water data: Information in support of the implementation of the Water Framework Directive.

Ross, P. E., \& Henebry, M. S. (1989). Use of four microbial tests to assess the ecological hazard of contaminated sediments. Toxicity Assessment, 4, 1-21.

Ryan, A.C., Tomasso, J.R., \& Klaine, S.J. (2009). Influence of pH, hardness, dissolved organic carbon concentration and dissolved organic matter source on the acute toxicity of copper to Daphnia magna in soft waters: Implications for the biotic ligand model. Environmental Toxicology and Chemistry, 28(8), 1663-1670.

Safe Drinking Water Committee. (1980). Drinking Water and Health (Vol. 2). Washington, D.C.: National Academy Press.

Samoiloff, M.R., Bell, J., Birkholz, D.A., Webster, G.R.B., Arnoff, E.G., Pulak, R., \& Madrid, A. (1983). Combined bioassay-chemical fractionation scheme for the determination and ranking of toxic chemicals in sediments. Environmental Science \& technology, 17(6), 329-334.

Sancho, E., Villarroel, M.J., Andreu, E., \& Ferrando, M.D. (2009). Disturbances in energy metabolism of Daphnia magna after exposure to tebuconazole. Chemosphere, 74, 1171-1178 
Sarma, S.S.S. \& Nandini, S. (2006). Review of recent ecotoxicological studies on cladocerans. Journal of Environmental Science and helath Part B, 41, 1417-1430.

Schaeffer, D.J. (1994). Detection - Bioassay. In Cockerham, L.G. \& Shane, B.S. (Eds.) Basic Environmental Toxicology. Boca Raton, FL: CRC Press.

Schmidt, K., Steinberg, C.E.W., Staaks, G.B.O. \& Pflugmacher, S. (2005). Influence of a xenobiotic mixture (PCB and TBT) compared to single substance on swimming behavior or reproduction of Daphnia magna. Acta Hydrochimica et Hydrobiologica, 33 (4), 287-300.

Schreiber, U., Quayle, P., Schmidt, S., Escher, B.I., \& Mueller, J.F. (2007). Methodology and evaluation of a highly sensitive algae toxicity test based on multiwell chlorophyll fluorescence imaging. Biosensors and Bioelectronics, 22, 2554-2563.

Schüürmann, G. (1997). Ecotoxic modes of action of chemical substances. In G. Schüürmann \& B. Markert (Eds.), Ecotoxicology: Ecological Fundamentals, Chemical Exposure, and Biological Effects. (pp. 665-749). New York: John Wiley \& Sons, Inc.

Semsari, S. \& Megateli, S. (2007). Effect of cadmium toxicity on survival and phototactic behaviour of Daphnia magna. Environmental Toxicology, 28, 799-806.

Shimizu, N., Ogino, C., Kawanishi, T., \& Hayashi, Y. (2002). Fractal analysis of Daphnia motion for acute toxicity bioassay. Environmental Toxicology, 17, 441448.

Snell, T.W. \& Janssen, C.R. (1995). Rotifers in ecotoxicology: a review. Hydrobiologia, 313/314, 231-247.

Snell, T.W. \& Joaquim-Justo, C. (2007). Workshop on rotifers in ecotoxicology. Hydrobiologia, 593, 227-232.

Staaks, G. \& Baganz, D. (2006). Aquatic animal behaviour test - video system tracks fish movements. Berlin, Germany: Leibniz-Institute of Freshwater Ecology and Inland Fisheries.

Storz, U.C. \& Paul, R.J. (1998). Phototaxis in water fleas (Daphnia magna) is differently indluenced by visible and UV light. Journal of Comparative Physioogy A, 183, 709-717.

Stratton, G.W. (1985). The influence of solvent type on solvent-pesticide interactions in bioassays. Archives of Environmental Contamination \& Toxicology, 14, 651-658.

Streb, C., Böcker, R. \& Häder, D.-P. (2006). Euglena gracilis: An indicator organism for aquatic toxicity of xenobiotics. Current Topics in Toxicology, 3: 17-24. 
Streb, C., Richter, P., Ntefidou, M., Lebert, M., \& Häder, D.-P. (2002). ECOTOX Biomonitoring based on real time movement analysis of unicellular organisms. Journal of Gravitational Physiology, 9(1): 345-346.

Tahedl, H. \& Häder, D.-P. (1999). Fast examination of water quality using the automatic biotest ECOTOX based on the movement behavior of a freshwater flagellate. Water Research, 33: 426-432.

Tahedl, H. \& Häder, D.-P. (2000). The use of image analysis in ecotoxicology, in D.-P. Häder (ed.) Image analysis methods and applications, CRC Press, Boca Raton, pp 447-458.

Tahedl, H. \& Häder, D.-P. (2001). Automated Biomonitoring Using Real Time Movement Analysis of Euglena gracilis. Ecotoxicology and Environmental Safety, 48: 161-169.

Tian, W., Meidong, H., \& Xingxin, Z. (2010, July 16). Contaminated waters that kill. China Daily. Retrieved on July 20, 2010, from http://www.chinadaily.com.cn/ cndy/2010-07/16/content_10113959.htm

United Nations Environment Programme (2006). Draft decision guidance document for tributyltin compounds. Rotterdam Convention on the Prior Informed Consent Procedure for Certain Hazardous Chemicals and Pesticides in International Trade Chemical Review Committee. Accessed on July 22, 2010, from http://www.pic.int/incs/crc3/n14\%29/English/K0654009\%20CRC3-14.pdf

Untersteiner, H., Kahapka. J., \& Kaiser, H. (2003). Behavioural response of the cladoceran Daphnia magna Straus to sublethal Copper stress - validation by image analysis. Aquatic Toxicology, 65, 435-442.

USEPA. (2002). Methods for Measuring Acute Toxicity of Effluents and Receiving waters to freshwater and marine organisms. Retrieved on July 10, 2010, from: http://www.epa.gov/waterscience/methods/wet/disk2/

Wang, F., Goulet, R.R. \& Chapman, P.M. (2004). Testing sediment biological effects with the freshwater amphipod Hyalella azteca: the gap between laboratory and nature. Chemosphere, 57, 1713-1724.

Watanabe, M., Henmi, K., Ogawa, K., \& Suzuki, T. (2003). Cadmium-dependent generation of reactive oxygen species and mitochondrial DNA breaks in photosynthetic and non-photosynthetic strains of Euglena gracilis. Comparative Biochemistry and Physiology part C, 134, 227-234.

Watson, S.B., Juttner, F. \& Koster, O. (2007) Daphnia behavioural responses to taste and odour compounds: ecological significance and application as an inline treatment plant monitoring tool. Water Science and Technology, 55, 23-31. 
Welsch, P.G., Lipton, J., Chapman, G.A., \& Podrabsky, T.L. (2000). Relative Importance of Calcium and Magnesium in Hardness-based Modification of Copper Toxicity. Environmental Toxicology and Chemistry, 19(6), 1624-1631.

Wenzel, A., Nendza, M., Hartmann, P., \& Kanne, R. (1997). Testbattery for the assessment of aquatic toxicity. Chemosphere, 35, 307-322.

Wetzel, R.G. (2001). Limnology: Lake and River Ecosystems, $3^{\text {rd }}$ ed. San Diego, CA: Elsevier Science.

Whitton, B.A. (1965). Extracellular Products of Blue-Green Algae. Journal of General Microbiology, 40, 1-11.

Winter, J. (2000). Effects of Golf Course Construction and Operation on the Aquatic Environment of the Precambrian Shield. Trent University Golf Course Research Group and the Ontario Ministry of the Environment. Retrieved on July 23, 2010, from: http://www.chebucto.ns.ca/ccn/info/Science/SWCS/INFO/GOLFCOURSES/winter_golf.html

Wolf, G., Scheunders, P., \& Selens, M. (1998). Evaluation of the swimming activity of Daphnia magna by image analysis after administration of sublethal Cadmium concentrations. Comparative Biochemistry and Physiology Part A, 120, 99-105.

World Health Organization (2004). Copper in Drinking-water: Background document for development of WHO Guidelines for Drinking-water Quality. Geneva: World Health Organization.

Zhou, Q., Zhang, J., Fu, J., Shi, J., \& Jiang, G. (2008). Biomonitoring: An appealing tool for assessment of metal pollution in the aquatic ecosystem. Analytica Chimica Acta, 606, 135-150. 


\section{Appendices}

Appendix A - Nutrient concentration of liquid mineral medium for E. gracilis culturing.

\begin{tabular}{|c|c|c|c|}
\hline COMPOUND & AMOUNT PER LITRE & \multicolumn{2}{|c|}{ STOCK SOLUTION } \\
\hline $\mathrm{C}_{2} \mathrm{H}_{3} \mathrm{NaO}_{2} \cdot 3 \mathrm{H}_{2} \mathrm{O}$ & $1 \mathrm{~g}$ & \multirow{5}{*}{ 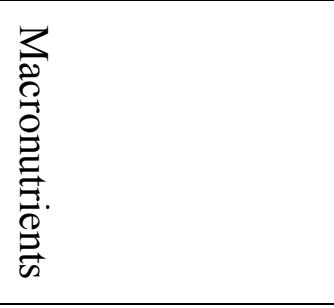 } & \\
\hline$\left(\mathrm{NH}_{4}\right)_{2} \mathrm{HPO}_{4}$ & $1 \mathrm{~g}$ & & \\
\hline $\mathrm{KH}_{2} \mathrm{PO}_{4}$ & $1 \mathrm{~g}$ & & \\
\hline $\mathrm{MgSO}_{4} \cdot 7 \mathrm{H}_{2} \mathrm{O}$ & $0.2 \mathrm{~g}$ & & \\
\hline $\mathrm{CaCl}_{2} \cdot 2 \mathrm{H}_{2} \mathrm{O}$ & $0.02 \mathrm{~g}$ & & \\
\hline Trace elements & $10 \mathrm{~mL}$ stock solution & \multirow{7}{*}{$\begin{array}{c}\mathrm{ZnSO}_{4} \cdot 7 \mathrm{H}_{2} \mathrm{O} \\
\mathrm{MnSO}_{4} \cdot 1 \mathrm{H}_{2} \mathrm{O} \\
\mathrm{Na}_{2} \mathrm{MoO}_{4} \cdot 2 \mathrm{H}_{2} \mathrm{O} \\
\mathrm{CoCl}_{2} \cdot 6 \mathrm{H}_{2} \mathrm{O} \\
\mathrm{CuSO}_{4} \cdot 5 \mathrm{H}_{2} \mathrm{O} \\
\mathrm{H}_{3} \mathrm{BO}_{3} \\
\mathrm{NaI}\end{array}$} & \multirow{7}{*}{$\begin{array}{c}4.4 \mathrm{~g} / \mathrm{L} \\
3.09 \mathrm{~g} / \mathrm{L} \\
1.008 \mathrm{~g} / \mathrm{L} \\
0.0807 \mathrm{~g} / \mathrm{L} \\
0.039 \mathrm{~g} / \mathrm{L} \\
0.0283 \mathrm{~g} / \mathrm{L} \\
0.0012 \mathrm{~g} / \mathrm{L}\end{array}$} \\
\hline & & & \\
\hline & & & \\
\hline & & & \\
\hline & & & \\
\hline & & & \\
\hline & & & \\
\hline $\mathrm{FeCl}_{3} \cdot 6 \mathrm{H}_{2} \mathrm{O}$ & $1 \mathrm{~mL}$ stock solution & $\mathrm{FeCl}_{3} \cdot 6 \mathrm{H}_{2} \mathrm{O}$ & $5 \mathrm{~g} / \mathrm{L}$ \\
\hline Thiamine (Vit. B1) & $1 \mathrm{~mL}$ stock solution & Vitamin B1 & $1 \mathrm{~g} / \mathrm{L}$ \\
\hline Cobalamin (Vit. B12) & $1 \mathrm{~mL}$ stock solution & Vitamin B12 & $1 \mathrm{~g} / \mathrm{L}$ \\
\hline
\end{tabular}


Appendix B - Calculation of water hardness for Daphnia magna culture water and Euglena gracilis culture medium.

Water hardness measured in ppm $(\mathrm{mg} / \mathrm{L}) \mathrm{CaCO}_{3}$

$$
\left[\mathrm{CaCO}_{3}\right]=\frac{M_{\mathrm{CaCO}_{3}}}{M_{\mathrm{Ca}}} \times\left[\mathrm{Ca}^{2+}\right]+\frac{M_{\mathrm{CaCO}_{3}}}{M_{\mathrm{Mg}}} \times\left[\mathrm{Mg}^{2+}\right]
$$

\section{Where}

$\mathrm{M}\left(\mathrm{CaCO}_{3}\right)=100.1$

$\mathrm{M}(\mathrm{Ca})=40.1$

$\mathrm{M}(\mathrm{Mg})=24.3$

For Daphnia magna growth culture water (Selection brand Natural Spring Water):

$[\mathrm{Ca}]=105 \mathrm{ppm}$

$[\mathrm{Mg}]=32.4 \mathrm{ppm}$

Then

$\left[\mathrm{CaCO}_{3}\right]=395 \mathrm{ppm}$

For Euglena gracilis mineral growth medium (Checcucci et al., 1976)

$\left[\mathrm{CaCl}_{2} \cdot 2 \mathrm{H}_{2} \mathrm{O}\right]=0.02 \mathrm{~g} / \mathrm{L}$ hence $[\mathrm{Ca}]=5.45 \mathrm{mg} / \mathrm{L}$ or ppm

$\left[\mathrm{MgSO}_{4} \cdot 7 \mathrm{H}_{2} \mathrm{O}\right]=0.2 \mathrm{~g} / \mathrm{L}$ hence $[\mathrm{Mg}]=19.7 \mathrm{mg} / \mathrm{L}$ or ppm

Then

$\left[\mathrm{CaCO}_{3}\right]=94.4 \mathrm{ppm}$ 
Appendix C - Nutrient concentration of liquid growth medium for P. subcapitata culturing.

\begin{tabular}{|c|l|c|c|}
\hline Stock & \multicolumn{1}{|c|}{ Compound } & $\begin{array}{c}\text { Quantity per 500 mL } \\
\text { stock }\end{array}$ & $\begin{array}{c}\text { Final concentration in growth } \\
\text { medium }\end{array}$ \\
\hline \multirow{2}{*}{1} & $\mathrm{NaNO}_{3}$ & $12.75 \mathrm{~g}$ & $25.5 \mathrm{mg} / \mathrm{L}$ \\
\hline 2 & $\mathrm{MgCl}_{2} \cdot 6 \mathrm{H}_{2} \mathrm{O}$ & $5.0 \mathrm{~g}$ & $10.0 \mathrm{mg} / \mathrm{L}$ \\
\cline { 2 - 4 } & $\mathrm{CaCl}_{2} \cdot 2 \mathrm{H}_{2} \mathrm{O}$ & $2.21 \mathrm{~g}$ & $4.42 \mathrm{mg} / \mathrm{L}$ \\
\cline { 2 - 4 } & $\mathrm{H}_{3} \mathrm{BO}_{3}$ & $92.76 \mathrm{mg}$ & $185.52 \mu \mathrm{g} / \mathrm{L}$ \\
\cline { 2 - 4 } & $\mathrm{MnCl}_{2} \cdot 4 \mathrm{H}_{2} \mathrm{O}$ & $207.81 \mathrm{mg}$ & $415.62 \mu \mathrm{g} / \mathrm{L}$ \\
\cline { 2 - 4 } & $\mathrm{ZnCl}_{2}$ & $1.64 \mathrm{mg}$ & $3.28 \mu \mathrm{g} / \mathrm{L}$ \\
\cline { 2 - 4 } & $\mathrm{CoCl}_{2} \cdot 6 \mathrm{H}_{2} \mathrm{O}$ & $0.714 \mathrm{mg}$ & $1.43 \mu \mathrm{g} / \mathrm{L}$ \\
\cline { 2 - 4 } & $\mathrm{CuCl}_{2} \cdot 2 \mathrm{H}_{2} \mathrm{O}$ & $0.006 \mathrm{mg}$ & $0.012 \mu \mathrm{g} / \mathrm{L}$ \\
\cline { 2 - 4 } & $\mathrm{Na}_{2} \mathrm{MoO}_{4} \cdot 2 \mathrm{H}_{2} \mathrm{O}$ & $3.63 \mathrm{mg}$ & $7.26 \mu \mathrm{g} / \mathrm{L}$ \\
\cline { 2 - 4 } & $\mathrm{FeCl}_{3} \cdot 6 \mathrm{H}_{2} \mathrm{O}$ & $80.0 \mathrm{mg}$ & $160 \mu \mathrm{g} / \mathrm{L}$ \\
\cline { 2 - 5 } & $\mathrm{Na}_{2} \mathrm{EDTA}_{2} \cdot 2 \mathrm{H}_{2} \mathrm{O}$ & $150.0 \mathrm{mg}$ & $300 \mu \mathrm{g} / \mathrm{L}$ \\
\hline 3 & $\mathrm{MgSO}_{4} \cdot 7 \mathrm{H}_{2} \mathrm{O}$ & $7.35 \mathrm{~g}$ & $14.7 \mathrm{mg} / \mathrm{L}$ \\
\hline 4 & $\mathrm{~K}_{2} \mathrm{HPO}_{4}$ & $0.522 \mathrm{~g}$ & $1.04 \mathrm{mg} / \mathrm{L}$ \\
\hline 5 & $\mathrm{NaHCO}_{3}$ & $7.5 \mathrm{~g}$ & $15 \mathrm{mg} / \mathrm{L}$ \\
\hline
\end{tabular}




\section{Appendix D - Nutrient concentration of COMBO growth medium for culturing of}

rotifers.

\begin{tabular}{|c|c|c|c|}
\hline Compound/Stock sol. & Final amount in medium & \multicolumn{2}{|c|}{ Concentration in stock } \\
\hline $\mathrm{CaCl}_{2} \cdot 2 \mathrm{H}_{2} \mathrm{O}$ & $36.76 \mathrm{mg} / \mathrm{L}$ (1 mL stock) & \multicolumn{2}{|c|}{$36.76 \mathrm{~g} / \mathrm{L}$} \\
\hline $\mathrm{MgSO}_{4} \cdot 7 \mathrm{H}_{2} \mathrm{O}$ & $36.97 \mathrm{mg} / \mathrm{L}$ (1 mL stock $)$ & \multicolumn{2}{|c|}{$36.97 \mathrm{~g} / \mathrm{L}$} \\
\hline $\mathrm{K}_{2} \mathrm{HPO}_{4}$ & $8.71 \mathrm{mg} / \mathrm{L}$ (1 mL stock) & \multicolumn{2}{|c|}{$8.71 \mathrm{~g} / \mathrm{L}$} \\
\hline $\mathrm{NaNO}_{3}$ & $85.01 \mathrm{mg} / \mathrm{L}(1 \mathrm{~mL}$ stock $)$ & \multicolumn{2}{|c|}{$85.01 \mathrm{~g} / \mathrm{L}$} \\
\hline $\mathrm{NaHCO}_{3}$ & $12.60 \mathrm{mg} / \mathrm{L}(1 \mathrm{~mL}$ stock $)$ & \multicolumn{2}{|c|}{$12.60 \mathrm{~g} / \mathrm{L}$} \\
\hline $\mathrm{Na}_{2} \mathrm{SiO}_{3} \cdot 9 \mathrm{H}_{2} \mathrm{O}$ & $28.42 \mathrm{mg} / \mathrm{L}$ (1 mL stock $)$ & \multicolumn{2}{|c|}{$28.42 \mathrm{~g} / \mathrm{L}$} \\
\hline $\mathrm{H}_{3} \mathrm{BO}_{3}$ & $24.00 \mathrm{mg} / \mathrm{L}(1 \mathrm{~mL}$ stock $)$ & \multicolumn{2}{|c|}{$24.00 \mathrm{~g} / \mathrm{L}$} \\
\hline $\mathrm{KCl}$ & $7.45 \mathrm{mg} / \mathrm{L}$ (1 mL stock) & \multicolumn{2}{|c|}{$7.45 \mathrm{~g} / \mathrm{L}$} \\
\hline \multirow[t]{7}{*}{ Algae trace elements } & \multirow[t]{7}{*}{$1 \mathrm{~mL}$ stock } & $\mathrm{Na}_{2}$ EDTA $\cdot 2 \mathrm{H}_{2} \mathrm{O}$ & $4.36 \mathrm{~g} / \mathrm{L}$ \\
\hline & & $\mathrm{FeCl}_{3} \cdot \mathrm{H}_{2} \mathrm{O}$ & $1.0 \mathrm{~g} / \mathrm{L}$ \\
\hline & & $\mathrm{MnCl}_{2} \cdot 4 \mathrm{H}_{2} \mathrm{O}$ & $180 \mathrm{mg} / \mathrm{L}$ \\
\hline & & $\mathrm{CuSO}_{4} \cdot 5 \mathrm{H}_{2} \mathrm{O}$ & $1 \mathrm{mg} / \mathrm{L}$ \\
\hline & & $\mathrm{Zn} \mathrm{SO}_{4} \cdot 7 \mathrm{H}_{2} \mathrm{O}$ & $22 \mathrm{mg} / \mathrm{L}$ \\
\hline & & $\mathrm{CoCl}_{2} \cdot 6 \mathrm{H}_{2} \mathrm{O}$ & $10 \mathrm{mg} / \mathrm{L}$ \\
\hline & & $\mathrm{Na}_{2} \mathrm{MoO}_{4} \cdot 2 \mathrm{H}_{2} \mathrm{O}$ & $22 \mathrm{mg} / \mathrm{L}$ \\
\hline \multirow[t]{3}{*}{ Animal trace elements } & \multirow[t]{3}{*}{$1 \mathrm{~mL}$ stock } & $\mathrm{LiCl}$ & $310 \mathrm{mg} / \mathrm{L}$ \\
\hline & & $\mathrm{NaBr}$ & $16 \mathrm{mg} / \mathrm{L}$ \\
\hline & & $\mathrm{KI}$ & $3.3 \mathrm{mg} / \mathrm{L}$ \\
\hline \multirow[t]{2}{*}{ Vitamin stock } & \multirow[t]{2}{*}{$0.5 \mathrm{~mL}$ stock } & Thiamine (B1) & $200 \mathrm{mg} / \mathrm{L}$ \\
\hline & & Cobalamin (B12) & $1.12 \mathrm{mg} / \mathrm{L}$ \\
\hline
\end{tabular}


Appendix E - Euglena gracilis percent motility after atrazine exposure - visual observation.

\begin{tabular}{|c|c|c|c|c|c|c|}
\hline & Time & Reference & $\begin{array}{l}0.1 \% \\
\text { DMSO } \\
\end{array}$ & $23.2 \mathrm{nM}$ & $231.8 \mathrm{nM}$ & $\begin{array}{l}2318.2 \\
\mathrm{nM}\end{array}$ \\
\hline Replicate 1 & $1 \mathrm{~h}$ & 58.551068 & 67.12967 & 40.67777 & 50.792208 & 52.878788 \\
\hline Replicate 2 & $1 \mathrm{~h}$ & 46.466667 & 48.002381 & 54.60714 & 59.07619 & 53.755556 \\
\hline Replicate 3 & $1 \mathrm{~h}$ & 61.772028 & 50.818182 & 58.17727 & 66.872711 & 59.581385 \\
\hline Replicate 4 & $1 \mathrm{~h}$ & 65.333333 & 72 & 64.83333 & 48.133333 & 40.166667 \\
\hline Replicate 5 & $1 \mathrm{~h}$ & 50.333333 & 46.9 & 54 & 28.466667 & 35.795238 \\
\hline Replicate 6 & $1 \mathrm{~h}$ & 56.182778 & 64.47619 & 54.16 & 50.670635 & 73.138095 \\
\hline Replicate 1 & $2 \mathrm{~h}$ & 63.12676 & 82.797222 & 63.57121 & 73.587899 & 74.166667 \\
\hline Replicate 2 & $2 \mathrm{~h}$ & 68.25 & 61.921429 & 50.66667 & 66.666667 & 56.022222 \\
\hline Replicate 3 & $2 \mathrm{~h}$ & 48.52381 & 55.315152 & 63.70363 & 61.472894 & 56.453102 \\
\hline Replicate 4 & $2 \mathrm{~h}$ & 76.166667 & 73.133333 & 67 & 73.1891 & 70.022222 \\
\hline Replicate 5 & $2 \mathrm{~h}$ & 68.333333 & 81.752381 & 85.8 & 82.5 & 79.022222 \\
\hline Replicate 6 & $2 \mathrm{~h}$ & 90.166667 & 81.333333 & 76.47619 & 82.177778 & 71.967521 \\
\hline Replicate 1 & $24 \mathrm{~h}$ & 48 & 40.6781 & 36.20317 & 51.833333 & 45 \\
\hline Replicate 2 & $24 \mathrm{~h}$ & 46.181818 & 40.07326 & 54.77778 & 43.730159 & 54.242424 \\
\hline Replicate 3 & $24 \mathrm{~h}$ & 50 & 40.5 & 31.61111 & 44.047619 & 53.623377 \\
\hline Replicate 4 & $24 \mathrm{~h}$ & 46.181818 & 40.250749 & 45.57143 & 41.250749 & 50.535714 \\
\hline Replicate 5 & $24 \mathrm{~h}$ & 36.487013 & 45.888889 & 37.5 & 44.5 & 41.611111 \\
\hline Replicate 6 & $24 \mathrm{~h}$ & 48 & 40.5 & 45.57143 & 43.730159 & 50.535714 \\
\hline
\end{tabular}


Appendix F - Euglena gracilis percent motility after TBT exposure - visual observation.

\begin{tabular}{|c|c|c|c|c|c|c|}
\hline & Time & Reference & $\begin{array}{l}0.1 \% \\
\text { DMSO }\end{array}$ & $1.7 \mathrm{nM}$ & $16.8 \mathrm{nM}$ & $167.9 \mathrm{nM}$ \\
\hline Replicate 1 & $1 \mathrm{~h}$ & 49.987471 & 50.35498 & 44.76523 & 48.08333 & 33.87179 \\
\hline Replicate 2 & $1 \mathrm{~h}$ & 41.990909 & 46.40476 & 30.45788 & 39.64286 & 41.37363 \\
\hline Replicate 3 & $1 \mathrm{~h}$ & 46.166667 & 59.88645 & 42.08333 & 56.78644 & 51.31025 \\
\hline Replicate 4 & $1 \mathrm{~h}$ & 41.873016 & 58.11722 & 47.46503 & 50.7619 & 46.91392 \\
\hline Replicate 5 & $1 \mathrm{~h}$ & 48.02381 & 52.15909 & 45.5 & 45.21032 & 44.88384 \\
\hline Replicate 1 & $2 \mathrm{~h}$ & 52.797619 & 54.99341 & 53.30635 & 56.88095 & 50.22161 \\
\hline Replicate 2 & $2 \mathrm{~h}$ & 39.863636 & 40.90801 & 31.41941 & 40.5 & 28.43573 \\
\hline Replicate 3 & $2 \mathrm{~h}$ & 54.45 & 54.30653 & 22.33333 & 60.26623 & 37.72222 \\
\hline Replicate 4 & $2 \mathrm{~h}$ & 48.580586 & 49.4359 & 46.16453 & 55.53968 & 38.61111 \\
\hline Replicate 5 & $2 \mathrm{~h}$ & 50.192308 & 48.56685 & 42.00855 & 54.35664 & 40.67932 \\
\hline Replicate 1 & $24 \mathrm{~h}$ & 35.961538 & 43.97619 & 35.34091 & 33.98701 & 35.6685 \\
\hline Replicate 2 & $24 \mathrm{~h}$ & 44.833333 & 46.4881 & 32.97619 & 22.54978 & 28.82857 \\
\hline Replicate 3 & $24 \mathrm{~h}$ & 52.5 & 47.82143 & 55.85714 & 42.32143 & 35.89011 \\
\hline Replicate 4 & $24 \mathrm{~h}$ & 48.333333 & 57.67857 & 43.83333 & 26 & 37.69841 \\
\hline Replicate 5 & $24 \mathrm{~h}$ & 42.371429 & 47.07576 & 47.11111 & 43.34343 & 27.95238 \\
\hline
\end{tabular}


Appendix G - Euglena gracilis percent motility after copper exposure - visual observation.

\begin{tabular}{|c|c|c|c|c|c|c|}
\hline & Time & Reference & $0.2 \mathrm{uM}$ & 0.4 uM & $2 \mathrm{uM}$ & 20 uM \\
\hline Replicate 1 & $1 \mathrm{~h}$ & 46.38132 & 33.93333 & 43.85238 & 5.755556 & 0.4 \\
\hline Replicate 2 & $1 \mathrm{~h}$ & 42.77381 & 29.05556 & 23.03636 & 6.4 & 0.8 \\
\hline Replicate 3 & $1 \mathrm{~h}$ & 52.58333 & 38.94048 & 54.4 & 13.7 & 1.1 \\
\hline Replicate 4 & $1 \mathrm{~h}$ & 31.41667 & 44.55556 & 42.56993 & 4.022222 & 0.2 \\
\hline Replicate 5 & $1 \mathrm{~h}$ & 45.47009 & 44.08485 & 59.4632 & 5.216667 & 0.458333 \\
\hline Replicate 6 & $1 \mathrm{~h}$ & 56.67063 & 40.51018 & 47.12381 & 8.490909 & 1.566667 \\
\hline Replicate 1 & $2 \mathrm{~h}$ & 47.16508 & 37.94286 & 38.30794 & 9.786724 & 2.4 \\
\hline Replicate 2 & $2 \mathrm{~h}$ & 46.65873 & 47.24545 & 59.64242 & 14.5 & 3.138889 \\
\hline Replicate 3 & $2 \mathrm{~h}$ & 53.06667 & 47.97222 & 37.22222 & 17.48291 & 3 \\
\hline Replicate 4 & $2 \mathrm{~h}$ & 43.02381 & 41.693 & 52.36818 & 4.064646 & 0.502331 \\
\hline Replicate 5 & $2 \mathrm{~h}$ & 50.92778 & 45.92308 & 59.92063 & 1.467677 & 0 \\
\hline Replicate 6 & $2 \mathrm{~h}$ & 45.70303 & 45.35455 & 37.60024 & 4 & 0.2 \\
\hline Replicate 1 & $24 \mathrm{~h}$ & 73.90897 & 57.12179 & 50.44805 & 72.97727 & 50.69444 \\
\hline Replicate 2 & $24 \mathrm{~h}$ & 59.79683 & 44.95714 & 57.5 & 65.25758 & 67.28636 \\
\hline Replicate 3 & $24 \mathrm{~h}$ & 58.06818 & 52.1 & 58.70859 & 55.89814 & 53.7803 \\
\hline Replicate 4 & $24 \mathrm{~h}$ & 36.63586 & 48.61111 & 45.39394 & 52.57446 & 25.9899 \\
\hline Replicate 5 & $24 \mathrm{~h}$ & 46.20833 & 48.25152 & 51.31818 & 49.62121 & 32.738 \\
\hline Replicate 6 & $24 \mathrm{~h}$ & 54.53968 & 44.77582 & 61.82078 & 60.32381 & 43.96061 \\
\hline
\end{tabular}


Appendix $\mathbf{H}$ - Euglena gracilis percent motility after copper exposure at different temperatures - visual observation.

\begin{tabular}{|c|c|c|c|c|c|c|c|}
\hline & $\begin{array}{l}\text { Temp } \\
{ }^{\circ} \mathrm{C}\end{array}$ & Time & $0.4 \mathrm{uM}$ & $0.8 \mathrm{uM}$ & $1.2 \mathrm{uM}$ & $1.6 \mathrm{uM}$ & $2 \mathrm{uM}$ \\
\hline Replicate 1 & 21 & $1 \mathrm{~h}$ & 45.68615 & 24.69444 & 26.91575 & 26.31457 & 12 \\
\hline Replicate 2 & 21 & $1 \mathrm{~h}$ & 37.69697 & 43.58333 & 21.03571 & 17.61905 & 19.06427 \\
\hline Replicate 3 & 21 & $1 \mathrm{~h}$ & 45.07143 & 29.3812 & 16.86429 & 15.05916 & 11.25794 \\
\hline Replicate 4 & 21 & $1 \mathrm{~h}$ & \begin{tabular}{|l|l|}
44.74881 \\
\end{tabular} & 33.67857 & 35.53687 & 17.64286 & 10.55556 \\
\hline \begin{tabular}{|l|} 
Replicate 1 \\
\end{tabular} & 21 & $2 \mathrm{~h}$ & 48.04762 & 26.66667 & 31.02381 & 27.14286 & 13.55556 \\
\hline Replicate 2 & 21 & $2 \mathrm{~h}$ & 42.96216 & 27.21795 & 33.16142 & 33.34343 & 19.34848 \\
\hline \begin{tabular}{|l} 
Replicate 3 \\
\end{tabular} & 21 & $2 \mathrm{~h}$ & 47.88254 & 26.80159 & 18.58874 & 9.194444 & 6.695238 \\
\hline Replicate 4 & 21 & $2 \mathrm{~h}$ & 48.70635 & 53.78571 & 25.91725 & 32.00794 & 17 \\
\hline \begin{tabular}{|l|} 
Replicate 1 \\
\end{tabular} & 26 & $1 \mathrm{~h}$ & 30.39394 & 21.01263 & 7.151515 & 3.592424 & 1.1 \\
\hline \begin{tabular}{|l} 
Replicate 2 \\
\end{tabular} & 26 & $1 \mathrm{~h}$ & 34.2803 & 26.10931 & 7.472222 & 3.256566 & 0.8 \\
\hline Replicate 3 & 26 & $1 \mathrm{~h}$ & 37.99567 & 19.55128 & 23.97727 & 7.464646 & 4.266667 \\
\hline \begin{tabular}{|l|} 
Replicate 4 \\
\end{tabular} & 26 & $1 \mathrm{~h}$ & 32.95238 & 28.66667 & 24.5404 & 9.702381 & 3.922222 \\
\hline Replicate 5 & 26 & $1 \mathrm{~h}$ & 32.42857 & 18.75 & 12.40152 & 5.270202 & 3.044444 \\
\hline \begin{tabular}{|l} 
Replicate 1 \\
\end{tabular} & 26 & $2 \mathrm{~h}$ & 33 & 12.36111 & 13.31667 & 4.666667 & \begin{tabular}{|l}
1.793651 \\
\end{tabular} \\
\hline Replicate 2 & 26 & $2 \mathrm{~h}$ & 40.71828 & 17.52778 & 7.594444 & 7.633333 & 1.894444 \\
\hline Replicate 3 & 26 & $2 \mathrm{~h}$ & 43.9798 & 20.97319 & 6.583333 & 5.966667 & \begin{tabular}{|l}
1.972222 \\
\end{tabular} \\
\hline Replicate 4 & 26 & $2 \mathrm{~h}$ & 30.36364 & 10.55556 & 12.61072 & 6.333333 & 3.8 \\
\hline Replicate 5 & 26 & $2 \mathrm{~h}$ & 40.71212 & 9.400794 & 12.29853 & 5.252381 & 2.407143 \\
\hline
\end{tabular}


Appendix I - Euglena gracilis cell shape after copper exposure

\begin{tabular}{|c|c|c|c|c|c|c|}
\hline & Time & Reference & $0.4 \mathrm{uM}$ & $1.2 \mathrm{uM}$ & $2 \mathrm{uM}$ & $20 \mathrm{uM}$ \\
\hline Replicate 1 & $1 \mathrm{~h}$ & 1.583333 & 1.5 & 1.416667 & 0.833333 & 0.5 \\
\hline Replicate 2 & $1 \mathrm{~h}$ & 1.166667 & 1.416667 & 1.5 & 1.083333 & 0.666667 \\
\hline Replicate 3 & $1 \mathrm{~h}$ & 1.833333 & 1.5 & 1.083333 & 1.083333 & 0.5 \\
\hline Replicate 1 & $2 \mathrm{~h}$ & 1.583333 & 1.75 & 1.416667 & 1.416667 & 1.083333 \\
\hline Replicate 2 & $2 \mathrm{~h}$ & 1.75 & 1.666667 & 1.5 & 1.083333 & 0.833333 \\
\hline Replicate 3 & $2 \mathrm{~h}$ & 1.583333 & 1.666667 & 1.25 & 1.25 & 1.083333 \\
\hline
\end{tabular}


Appendix $\mathbf{J}$ - Euglena gracilis automated parameters measured by ECOTOX after atrazine exposure.

A. Cell swimming velocity

\begin{tabular}{|l|l|r|r|r|r|}
\cline { 2 - 6 } \multicolumn{1}{c|}{} & Time & Reference & $\mathbf{2 3 . 2} \mathbf{~ n M}$ & $\begin{array}{l}\mathbf{2 3 1 . 8} \\
\mathbf{n M}\end{array}$ & $\begin{array}{l}\mathbf{2 3 1 8 . 2} \\
\text { nM }\end{array}$ \\
\hline Replicate 1 & $2 \mathrm{~h}$ & 139.268 & 185.9729 & 188.3314 & 182.36 \\
\hline Replicate 2 & $2 \mathrm{~h}$ & 152.2965 & 173.76 & 175.9874 & 182.0325 \\
\hline Replicate 3 & $2 \mathrm{~h}$ & 187.4056 & 172.2958 & 194.8827 & 184.3043 \\
\hline Replicate 4 & $2 \mathrm{~h}$ & 176.7793 & 180.3807 & 181.319 & 174.7824 \\
\hline Replicate 1 & $24 \mathrm{~h}$ & 137.0518 & 142.9966 & 100.1626 & 143.5624 \\
\hline Replicate 2 & $24 \mathrm{~h}$ & 138.4509 & 151.1188 & 143.5624 & 131.0092 \\
\hline Replicate 3 & $24 \mathrm{~h}$ & 140.5676 & 163.2381 & 152.6715 & 153.1711 \\
\hline Replicate 4 & $24 \mathrm{~h}$ & 137.6464 & 138.9907 & 110.1249 & 125.6823 \\
\hline
\end{tabular}

B. Cell shape (form factor)

\begin{tabular}{|l|l|r|r|r|r|}
\cline { 2 - 6 } \multicolumn{1}{c|}{} & Time & Reference & $\mathbf{2 3 . 2} \mathbf{~ n M}$ & $\begin{array}{l}\mathbf{2 3 1 . 8} \\
\mathbf{n M}\end{array}$ & $\begin{array}{l}\mathbf{2 3 1 8 . 2} \\
\mathbf{n M}\end{array}$ \\
\hline Replicate 1 & 2h & 4.780332 & 5.553055 & 5.586608 & 5.462225 \\
\hline Replicate 2 & $2 \mathrm{~h}$ & 4.742271 & 5.396921 & 5.040452 & 5.291232 \\
\hline Replicate 3 & $2 \mathrm{~h}$ & 5.726323 & 5.085725 & 5.67409 & 5.557692 \\
\hline Replicate 4 & $2 \mathrm{~h}$ & 5.286798 & 5.642184 & 5.287033 & 5.333112 \\
\hline Replicate 1 & $24 \mathrm{~h}$ & 4.978818 & 4.283556 & 3.39728 & 4.5731 \\
\hline Replicate 2 & $24 \mathrm{~h}$ & 4.795125 & 4.589709 & 4.49266 & 4.179308 \\
\hline Replicate 3 & $24 \mathrm{~h}$ & 4.391701 & 4.49266 & 4.624046 & 4.73071 \\
\hline Replicate 4 & $24 \mathrm{~h}$ & 4.558674 & 4.276601 & 3.844812 & 4.615047 \\
\hline
\end{tabular}


C. Percent cells swimming up

\begin{tabular}{|c|c|c|c|c|c|}
\hline & Time & Reference & $23.2 \mathrm{nM}$ & $\begin{array}{l}231.8 \\
\mathrm{nM}\end{array}$ & $\begin{array}{l}2318.2 \\
\mathrm{nM}\end{array}$ \\
\hline Replicate 1 & $2 \mathrm{~h}$ & 39.32719 & 29.4 & 33.4 & 29.70098 \\
\hline Replicate 2 & $2 \mathrm{~h}$ & 48.45794 & 34.34646 & 32.36747 & 35.52727 \\
\hline Replicate 3 & $2 \mathrm{~h}$ & 34.71713 & 31.31868 & 33.81991 & 30.63462 \\
\hline Replicate 4 & $2 \mathrm{~h}$ & 33.33333 & 31.67364 & 32.2623 & 37.72197 \\
\hline Replicate 1 & $24 \mathrm{~h}$ & 44.79679 & 38.44444 & 30.49 & 26.92222 \\
\hline Replicate 2 & $24 \mathrm{~h}$ & 42.6299 & 33.96203 & 40.15646 & 33.83178 \\
\hline Replicate 3 & $24 \mathrm{~h}$ & 46.84751 & 40.15646 & 30.24615 & 35.09091 \\
\hline Replicate 4 & $24 \mathrm{~h}$ & 52.37847 & 34.9899 & 22.18045 & 32.87549 \\
\hline
\end{tabular}

D. Randomness of swimming (r-value)

\begin{tabular}{|l|l|r|r|l|l|}
\cline { 2 - 6 } \multicolumn{1}{c|}{} & Time & Reference & $\mathbf{2 3 . 2} \mathbf{~ n M}$ & $\begin{array}{l}\mathbf{2 3 1 . 8} \\
\mathbf{n M}\end{array}$ & $\begin{array}{l}\mathbf{2 3 1 8 . 2} \\
\mathbf{n M}\end{array}$ \\
\hline Replicate 1 & $2 \mathrm{~h}$ & 0.141553 & 0.281545 & 0.261525 & 0.279549 \\
\hline Replicate 2 & $2 \mathrm{~h}$ & 0.303607 & 0.228504 & 0.225819 & 0.205536 \\
\hline Replicate 3 & $2 \mathrm{~h}$ & 0.223629 & 0.246885 & 0.251142 & 0.294346 \\
\hline Replicate 4 & $2 \mathrm{~h}$ & 0.232844 & 0.260556 & 0.215676 & 0.206547 \\
\hline Replicate 1 & $24 \mathrm{~h}$ & 0.153612 & 0.2549 & 0.22373 & 0.2649 \\
\hline Replicate 2 & $24 \mathrm{~h}$ & 0.150556 & 0.333532 & 0.221599 & 0.215009 \\
\hline Replicate 3 & $24 \mathrm{~h}$ & 0.192255 & 0.221599 & 0.226723 & 0.177136 \\
\hline Replicate 4 & $24 \mathrm{~h}$ & 0.224302 & 0.168773 & 0.19812 & 0.181008 \\
\hline
\end{tabular}


Appendix K - Euglena gracilis automated parameters measured by ECOTOX after TBT exposure.

A. Cell swimming velocity

\begin{tabular}{|l|l|r|r|r|r|}
\cline { 2 - 6 } \multicolumn{1}{c|}{} & Time & Reference & $\mathbf{1 . 7} \mathbf{n M}$ & $\mathbf{1 6 . 8} \mathbf{~ n M}$ & $\begin{array}{l}\mathbf{1 6 7 . 9} \\
\text { nM }\end{array}$ \\
\hline Replicate 1 & $2 \mathrm{~h}$ & 139.268 & 83.85371 & 189.15 & 189.4306 \\
\hline Replicate 2 & $2 \mathrm{~h}$ & 152.2965 & 182.4195 & 185.8835 & 180.185 \\
\hline Replicate 3 & $2 \mathrm{~h}$ & 187.4056 & 186.4314 & 198.3887 & 184.5063 \\
\hline Replicate 4 & $2 \mathrm{~h}$ & 176.7793 & 192.2022 & 198.7872 & 175.0032 \\
\hline Replicate 1 & $24 \mathrm{~h}$ & 137.0518 & 113.2425 & 149.0291 & 147.2964 \\
\hline Replicate 2 & $24 \mathrm{~h}$ & 138.4509 & 164.0077 & 125.2334 & 157.2084 \\
\hline Replicate 3 & $24 \mathrm{~h}$ & 140.5676 & 146.899 & 142.1433 & 148.4566 \\
\hline Replicate 4 & $24 \mathrm{~h}$ & 137.6464 & 152.63 & 157.707 & 142.2946 \\
\hline
\end{tabular}

B. Cell shape (form factor)

\begin{tabular}{|l|l|r|l|l|l|}
\cline { 2 - 6 } \multicolumn{1}{c|}{} & Time & Reference & $\mathbf{1 . 7} \mathbf{n M}$ & $\mathbf{1 6 . 8} \mathbf{~ n M}$ & $\begin{array}{l}\mathbf{1 6 7 . 9} \\
\text { nM }\end{array}$ \\
\hline Replicate 1 & 2h & 4.780332 & 5.427477 & 5.561303 & 5.888996 \\
\hline Replicate 2 & $2 \mathrm{~h}$ & 4.742271 & 5.099914 & 5.608518 & 5.898645 \\
\hline Replicate 3 & $2 \mathrm{~h}$ & 5.726323 & 5.483985 & 5.722688 & 5.800951 \\
\hline Replicate 4 & $2 \mathrm{~h}$ & 5.286798 & 5.780261 & 5.689343 & 5.082991 \\
\hline Replicate 1 & $24 \mathrm{~h}$ & 4.978818 & 3.951593 & 4.576829 & 4.584083 \\
\hline Replicate 2 & $24 \mathrm{~h}$ & 4.795125 & 5.130467 & 4.004327 & 5.065881 \\
\hline Replicate 3 & $24 \mathrm{~h}$ & 4.391701 & 4.412711 & 4.950573 & 4.996249 \\
\hline Replicate 4 & $24 \mathrm{~h}$ & 4.558674 & 4.811606 & 4.991943 & 4.699843 \\
\hline
\end{tabular}


C. Percent cells swimming up

\begin{tabular}{|l|l|r|r|r|l|}
\cline { 2 - 6 } \multicolumn{1}{c|}{} & Time & Reference & $\mathbf{1 . 7} \mathbf{n M}$ & $\mathbf{1 6 . 8} \mathbf{n M}$ & $\begin{array}{l}\mathbf{1 6 7 . 9} \\
\text { nM }\end{array}$ \\
\hline Replicate 1 & $2 \mathrm{~h}$ & 39.32719 & 19.54955 & 32.14938 & 28.36187 \\
\hline Replicate 2 & $2 \mathrm{~h}$ & 48.45794 & 31.36 & 29.43077 & 29.46565 \\
\hline Replicate 3 & $2 \mathrm{~h}$ & 34.71713 & 31.1283 & 32.32813 & 36.68137 \\
\hline Replicate 4 & $2 \mathrm{~h}$ & 33.33333 & 29.35915 & 36.77509 & 31.40752 \\
\hline Replicate 1 & $24 \mathrm{~h}$ & 44.79679 & 27.26207 & 31.29282 & 39.76111 \\
\hline Replicate 2 & $24 \mathrm{~h}$ & 42.6299 & 33.42857 & 34.79762 & 39.67925 \\
\hline Replicate 3 & $24 \mathrm{~h}$ & 46.84751 & 31.20805 & 29.72332 & 33.59391 \\
\hline Replicate 4 & $24 \mathrm{~h}$ & 52.37847 & 31.83871 & 31.78261 & 35.5814 \\
\hline
\end{tabular}

D. Randomness of swimming (r-value)

\begin{tabular}{|l|l|r|r|l|l|}
\cline { 2 - 6 } \multicolumn{1}{c|}{} & Time & Reference & $\mathbf{1 . 7} \mathbf{n M}$ & $\mathbf{1 6 . 8} \mathbf{n M}$ & $\begin{array}{l}\mathbf{1 6 7 . 9} \\
\text { nM }\end{array}$ \\
\hline Replicate 1 & $2 \mathrm{~h}$ & 0.141553 & 0.184613 & 0.260062 & 0.300004 \\
\hline Replicate 2 & $2 \mathrm{~h}$ & 0.303607 & 0.231629 & 0.318944 & 0.268206 \\
\hline Replicate 3 & $2 \mathrm{~h}$ & 0.223629 & 0.24263 & 0.278598 & 0.22677 \\
\hline Replicate 4 & $2 \mathrm{~h}$ & 0.232844 & 0.266764 & 0.217917 & 0.263545 \\
\hline Replicate 1 & $24 \mathrm{~h}$ & 0.153612 & 0.200869 & 0.195807 & 0.165311 \\
\hline Replicate 2 & $24 \mathrm{~h}$ & 0.150556 & 0.231362 & 0.172667 & 0.237013 \\
\hline Replicate 3 & $24 \mathrm{~h}$ & 0.192255 & 0.199597 & 0.146391 & 0.169848 \\
\hline Replicate 4 & $24 \mathrm{~h}$ & 0.224302 & 0.191587 & 0.197361 & 0.189006 \\
\hline
\end{tabular}


Appendix L - Euglena gracilis automated parameters measured by ECOTOX after copper exposure.

A. Cell swimming velocity

\begin{tabular}{|l|l|r|l|l|l|l|}
\cline { 2 - 7 } \multicolumn{1}{c|}{} & Time & Reference & $\mathbf{0 . 4} \mathbf{\mu M}$ & $\mathbf{1 . 2} \mathbf{\mu M}$ & $\mathbf{2 . 0} \mathbf{~ M}$ & $\mathbf{2 0 . 0} \boldsymbol{\mu M}$ \\
\hline Replicate 1 & 1h & 148.1637 & 129.8883 & 169.5628 & 158.7903 & 119.0959 \\
\hline Replicate 2 & 1h & 160.4029 & 152.0274 & 178.6311 & 156.8702 & 90.6906 \\
\hline Replicate 3 & 1h & 140.4901 & 152.9597 & 160.109 & 154.6701 & 72.11802 \\
\hline Replicate 4 & 1h & 164.6979 & 181.874 & 163.8374 & 166.9629 & 82.66069 \\
\hline Replicate 1 & 2h & 142.9274 & 176.5783 & 153.7118 & 152.1084 & 112.5434 \\
\hline Replicate 2 & 2h & 166.8761 & 189.4274 & 141.2754 & 126.2438 & 90.99183 \\
\hline Replicate 3 & 2h & 173.6657 & 158.8693 & 149.9568 & 104.9569 & 64.25036 \\
\hline Replicate 4 & 2h & 159.8753 & 185.4159 & 172.7057 & 140.4853 & 61.55912 \\
\hline
\end{tabular}

B. Cell shape (form factor)

\begin{tabular}{|l|l|r|l|l|l|r|}
\cline { 2 - 7 } \multicolumn{1}{c|}{} & Time & Reference & $\mathbf{0 . 4} \mathbf{\mu M}$ & $\mathbf{1 . 2} \mathbf{~ M}$ & $\mathbf{2 . 0} \mathbf{~ M}$ & $\mathbf{2 0 . 0} \boldsymbol{\mu M}$ \\
\hline Replicate 1 & 1h & 4.855874 & 4.225954 & 5.24994 & 5.239196 & 4.268 \\
\hline Replicate 2 & $1 \mathrm{~h}$ & 5.437524 & 5.105762 & 5.072658 & 5.022205 & 5.388 \\
\hline Replicate 3 & $1 \mathrm{~h}$ & 4.775413 & 4.624447 & 5.214131 & 4.73482 & 5.375 \\
\hline Replicate 4 & $1 \mathrm{~h}$ & 5.785562 & 5.200583 & 4.629749 & 5.144739 & 4.632 \\
\hline Replicate 1 & $2 \mathrm{~h}$ & 5.477928 & 5.472977 & 5.079158 & 5.064942 & 4.365715 \\
\hline Replicate 2 & $2 \mathrm{~h}$ & 5.697992 & 5.825105 & 4.848316 & 4.310371 & 4.636028 \\
\hline Replicate 3 & $2 \mathrm{~h}$ & 6.058778 & 5.44343 & 5.082407 & 4.242145 & 3.83407 \\
\hline Replicate 4 & $2 \mathrm{~h}$ & 4.907782 & 5.534549 & 5.082387 & 4.677272 & 3.09178 \\
\hline
\end{tabular}


C. Percent cells swimming up

\begin{tabular}{|l|l|r|r|l|l|l|}
\cline { 2 - 7 } \multicolumn{1}{c|}{} & Time & Reference & $\mathbf{0 . 4} \mathbf{\mu M}$ & $\mathbf{1 . 2} \mathbf{~ M}$ & $\mathbf{2 . 0} \mathbf{~ M}$ & $\mathbf{2 0 . 0} \mathbf{~ M}$ \\
\hline Replicate 1 & $1 \mathrm{~h}$ & 44.62185 & 44.9084 & 26.44841 & 36.94845 & 34.01438 \\
\hline Replicate 2 & $1 \mathrm{~h}$ & 37.32647 & 35.68027 & 32.9901 & 35.6875 & 34.09178 \\
\hline Replicate 3 & $1 \mathrm{~h}$ & 44.78864 & 32.19417 & 32.65421 & 29.52096 & 27.26 \\
\hline Replicate 4 & $1 \mathrm{~h}$ & 43.51698 & 27.28571 & 35.51282 & 35.21809 & 32.10438 \\
\hline Replicate 1 & $2 \mathrm{~h}$ & 37.07494 & 31.68182 & 31.86842 & 37.56494 & 27.27083 \\
\hline Replicate 2 & $2 \mathrm{~h}$ & 37.99479 & 30.22099 & 30.95918 & 30.77679 & 23.46897 \\
\hline Replicate 3 & $2 \mathrm{~h}$ & 39.23356 & 26.56395 & 32.85311 & 23.73092 & 20.93458 \\
\hline Replicate 4 & $2 \mathrm{~h}$ & 34.36 & 32.51634 & 31.03226 & 32.3237 & 18.13475 \\
\hline
\end{tabular}

D. Randomness of swimming (r-value)

\begin{tabular}{|l|l|r|l|l|l|l|}
\cline { 2 - 7 } \multicolumn{1}{c|}{} & Time & Reference & $\mathbf{0 . 4} \boldsymbol{\mu M}$ & $\mathbf{1 . 2} \boldsymbol{\mu M}$ & $\mathbf{2 . 0} \mathbf{\mu M}$ & $\mathbf{2 0 . 0} \boldsymbol{\mu M}$ \\
\hline Replicate 1 & $1 \mathrm{~h}$ & 0.158866 & 0.192431 & 0.253615 & 0.178716 & 0.223657 \\
\hline Replicate 2 & $1 \mathrm{~h}$ & 0.173132 & 0.263197 & 0.234079 & 0.206335 & 0.181834 \\
\hline Replicate 3 & $1 \mathrm{~h}$ & 0.200543 & 0.275214 & 0.190584 & 0.197665 & 0.277309 \\
\hline Replicate 4 & $1 \mathrm{~h}$ & 0.174491 & 0.310071 & 0.181631 & 0.211723 & 0.213269 \\
\hline Replicate 1 & $2 \mathrm{~h}$ & 0.168245 & 0.284318 & 0.20998 & 0.247727 & 0.190056 \\
\hline Replicate 2 & $2 \mathrm{~h}$ & 0.195089 & 0.264602 & 0.182061 & 0.222598 & 0.21729 \\
\hline Replicate 3 & $2 \mathrm{~h}$ & 0.191882 & 0.256 & 0.159944 & 0.148486 & 0.17721 \\
\hline Replicate 4 & $2 \mathrm{~h}$ & 0.173924 & 0.280098 & 0.300699 & 0.183566 & 0.176248 \\
\hline
\end{tabular}

